\title{
Critical analysis of the luminosity functions per galaxy type measured from redshift surveys
}

\author{
V. de Lapparent ${ }^{\star}$ \\ Institut d'Astrophysique de Paris, CNRS, Univ. Pierre et Marie Curie, 98bis boulevard Arago, 75014 Paris, France
}

Received 27 March 2003 / Accepted 12 June 2003

\begin{abstract}
I perform a quantitative comparison of the shape of the optical luminosity functions as a function of galaxy class and filter, which have been obtained from redshift surveys with an effective depth ranging from $z \simeq 0.01$ to $z \simeq 0.6$. This analysis is based on the $M^{*}$ and $\alpha$ Schechter parameters which are systematically measured for all galaxy redshift surveys. I provide complete tables of all the existing measurements, which I have converted into the $U B V R_{\mathrm{c}} I_{\mathrm{c}}$ Johnson-Cousins system wherever necessary.

By using as reference the intrinsic luminosity functions per morphological type, I establish that the variations in the luminosity functions from survey to survey and among the galaxy classes are closely related to the criteria for galaxy classification used in the surveys, as these determine the amount of mixing of the known morphological types within a given class. When using a spectral classification, the effect can be acute in the case of inaccurate spectrophotometric calibrations: the luminosity functions are then biased by type contamination and display a smooth variation from type to type which might be poorly related to the intrinsic luminosity functions per morphological type. In the case of surveys using multi-fiber spectroscopy, galaxy classification based on rest-frame colors might provide better estimates of the intrinsic luminosity functions.

It is noticeable that all the existing redshift surveys fail to measure the Gaussian luminosity function for Spiral galaxies, presumably due to contamination by dwarf galaxies. Most existing redshift surveys based on visual morphological classification also appear to have their Elliptical/Lenticular luminosity functions contaminated by dwarf galaxies. In contrast, the analyses using a reliable spectral classification based on multi-slit spectroscopy or medium-filter spectrophotometry, and combined with accurate CCD photometry succeed in measuring the Gaussian luminosity function for E/S0 galaxies. The present analysis therefore calls for a more coherent approach in separating the relevant giant and dwarf galaxy types, a necessary step towards measuring reliable intrinsic luminosity functions.
\end{abstract}

Key words. galaxies: fundamental parameters - galaxies: luminosity function, mass function galaxies: elliptical and lenticular, cD - galaxies: spiral - galaxies: irregular - galaxies: dwarf

\section{Introduction}

Among the fundamental characteristics of galaxies is their luminosity function (LF hereafter). In the current models of galaxy formation based on gravitational clustering, the LF provides constraints on the formation of galaxies within the dark matter halos (Cole et al. 2000; Baugh et al. 2002), thus allowing one to adjust the parameters for star formation, feedback processes, and mergers within the halos. Based on assumptions about the formation of bulge-dominated and disk-dominated galaxies, the various galaxy types can be traced separately in the models, which enables one to perform direct comparison with the observations (Baugh et al. 1996; Kauffmann et al. 1997; Cole et al. 2000). The LF in infrared bands provides the best constraints as it reliably reflects the underlying stellar mass and is poorly sensitive to extinction and bursts of star formation (Kauffmann \& Charlot 1998). Comparison with both the

\footnotetext{
* e-mail: lapparen@iap.fr
}

optical and infrared LFs provides tight constraints on the models for galaxy formation (Baugh et al. 2002).

With the goal to derive observational measures of the galaxy LF, a wide variety of redshift surveys with photometry from the UV to the infrared have been analyzed. The galaxy LF is however best known in the optical, where a wealth of details is measured. The optical "general" LFs show variations from survey to survey (Efstathiou et al. 1988; Marzke et al. 1994b; Loveday et al. 1995; Ellis et al. 1996; Lin et al. 1996; Zucca et al. 1997; Marzke et al. 1998; Ratcliffe et al. 1998), which can be partly explained by the different selection criteria used in each survey. The large statistical samples provided by the optical redshift surveys have also allowed one to separately measure the LFs for different galaxy populations, and have revealed marked differences (Efstathiou et al. 1988; Loveday et al. 1992; Marzke et al. 1994a; Lilly et al. 1995; Lin et al. 1996; Heyl et al. 1997; Lin et al. 1997, 1999; Small et al. 1997; Zucca et al. 1997; Bromley et al. 1998; Marzke et al. 1998; Metcalfe et al. 1998; Folkes et al. 1999; Loveday et al. 1999; 
Marinoni et al. 1999; Brown et al. 2001; Fried et al. 2001; Madgwick et al. 2002).

In parallel, studies of local galaxy concentrations have provided detailed understanding of the galaxy LF, by showing that each morphological type has a distinct LF, denoted "intrinsic" LF, with different parametric functions for the giant and the dwarf galaxies (see the review by Binggeli et al. 1988). Sandage et al. (1985), Ferguson \& Sandage (1991), and Jerjen \& Tammann (1997) show that the giant galaxies have Gaussian LFs, with the LF for Elliptical galaxies skewed towards faint magnitudes; in contrast, the LFs for dwarf galaxies may be ever increasing at faint magnitudes to the limit of the existing surveys, with a steeper increase for the dwarf Elliptical galaxies (dE) compared to the dwarf Irregular galaxies (dI).

Despite a widely varying behavior of the intrinsic LFs at faint magnitudes for the different galaxy types, they conspire to produce in most redshift surveys a "general" LF with a flat, or nearly flat shape at faint magnitudes (see for example Geller et al. 1997; Loveday et al. 1992). Interpretation of the "general" LF is complex because it results from the combination of the intrinsic LFs with the relative proportion of galaxies in each galaxy class and in the various environments probed by the survey. For example, the relative density of giant galaxy types is a function of galaxy density (as measured in clusters and groups by the morphology-density relation, Dressler 1980; Postman \& Geller 1984). Density-dependent effects are also present in the dwarf galaxy LFs (Binggeli et al. 1990; Ferguson \& Sandage 1991; Trentham \& Hodgkin 2002). And the redshift surveys are known to probe regions with widely varying densities, like voids, groups, clusters, etc. (see de Lapparent et al. 1986; Ramella et al. 1990). It is therefore difficult to derive reliable constraints on the intrinsic LF for any given galaxy type from the sole knowledge of the "general" LF. As emphasized by Binggeli et al. (1988), a complete characterization of the "general" galaxy LF requires measurement of the intrinsic LFs for each galaxy population.

The key for a robust measure of the intrinsic LFs is to reliably separate the different galaxy morphological types. To this end, most of the redshift surveys have been submitted to some galaxy classification scheme. The "nearby" redshift surveys $(z \lesssim 0.1)$ are based on photographic catalogues, for which visual morphological classification has been obtained (Efstathiou et al. 1988; Loveday et al. 1992; Marzke et al. 1994a, 1998; Marinoni et al. 1999). These surveys however do not explicitly include the low surface brightness dSph (for dwarf Spheroidal, comprising $\mathrm{dE}$ and $\mathrm{dS} 0$ ) and dI galaxies detected in the surveys of local galaxy concentrations (Sandage et al. 1985; Ferguson \& Sandage 1991; Jerjen \& Tammann 1997). The recent morphological analysis of a sub-sample of the Sloan Digital Sky Survey (Nakamura et al. 2003) based on $\mathrm{CCD}$ imaging to $z \sim 0.1$ however shows evidence for a contribution from dwarf galaxies. At redshifts larger than $\sim 0.1$, visual morphological classification becomes highly uncertain and is replaced by spectral classification (Heyl et al. 1997; Bromley et al. 1998; Lin et al. 1999; Folkes et al. 1999; Fried et al. 2001; Madgwick et al. 2002; Wolf et al. 2003; de Lapparent et al. 2003b). Other redshifts surveys for which a spectral classification is not available use either colors (Lilly et al. 1995;
Lin et al. 1997; Metcalfe et al. 1998; Brown et al. 2001) or the strength of the emission lines (Lin et al. 1996; Small et al. 1997; Zucca et al. 1997; Loveday et al. 1999) for estimating the LFs of the different galaxy types. The widely varying criteria used for galaxy classification in systematic redshift surveys however complicate the interpretation and inter-comparison of the derived LFs.

In the following, I examine all the existing measurements of intrinsic LFs obtained from optical redshift surveys at $z \lesssim 0.6$, and I convert them into the Johnson-Cousins $U B V R_{\mathrm{c}} I_{\mathrm{c}}$ system when other photometric systems are used. This allows a homogeneous comparison of the intrinsic LFs measured in each band. Note that here, the denomination "redshift survey" means "systematic survey of a region of the sky wide enough to include both cluster/group galaxies and field galaxies, and for which estimates of redshifts are provided". I therefore include the surveys by Fried et al. (2001) and Wolf et al. (2003), based on medium-band photometric redshifts; both surveys provide useful estimates of intrinsic LFs, consistent with those from the other surveys in the $B$ band, which a posteriori justifies their inclusion into the analysis.

The article is organized as follows. In Sect. 2, I recall the properties of the intrinsic LFs based on galaxy morphological type derived from the nearby galaxy concentrations. In Sect. 3, I analyze all existing measurements of intrinsic LFs in the $U$ and $V$ Johnson bands (Sect. 3.1), in the $R_{\mathrm{c}}$ Cousins band (Sect. 3.2), in the B Johnson band (Sect. 3.3), and in the $I_{\mathrm{c}}$ Cousins band (Sect. 3.4). Section 3.5 comments on the relation between the intrinsic LFs and the "general" LF. Finally, Sect. 4 summarizes the salient results and discusses the prospects raised by the present analysis.

\section{The local luminosity functions per morphological type}

Throughout the following sections, I use the estimated shape of the intrinsic LFs per galaxy morphological type to interpret the measured LFs from redshift surveys. Such a comparison has the advantage to provide clues on the morphological types included in the various classes of the considered samples. de Lapparent et al. (2003b) have first emphasized the interest of this approach. The authors show that the $R_{\mathrm{c}} \mathrm{LFs}$ for the early, intermediate and late spectral classes of the ESO-Sculptor redshift survey can be successfully modeled as composites of the LFs measured locally for the known morphological types of giant and dwarf galaxies. The success in using this approach for a redshift survey prompts to extend it to the general comparison performed here.

Following the seminal paper by Sandage et al. (1985), which shows that the LFs of Elliptical, Lenticular and Spiral galaxies in the Virgo cluster are bounded at bright and faint magnitudes, Jerjen \& Tammann (1997) derive from the joint analysis of the Virgo, Centaurus, and Fornax clusters a robust determination of the parametric forms for the intrinsic LFs of giant galaxies: the LFs for S0 and Spiral galaxies have Gaussian shapes, and the LF for Elliptical galaxies is well fitted by a two-wing Gaussian (a Gaussian with two different dispersion wings at the bright and faint end), which is 
Table 1. Estimated parameters of the local Gaussian and Schechter LFs for the different morphological types, in the Johnson-Cousins $B, V$, and $R_{\mathrm{c}}$ bands.

\begin{tabular}{|c|c|c|c|c|c|c|c|}
\hline \multirow[t]{2}{*}{ Morph. type } & \multicolumn{3}{|c|}{ Gaussian $M_{0}-5 \log h$} & \multirow[t]{2}{*}{ Gaussian $\Sigma$} & \multicolumn{3}{|c|}{$\phi_{0}{ }^{\mathrm{a}}$} \\
\hline & $R_{\mathrm{c}}^{\mathrm{b}}$ & $V^{\mathrm{b}}$ & $B^{\mathrm{b}}$ & & $R_{\mathrm{c}}$ & V & $B$ \\
\hline $\mathrm{E}$ & $-20.0 \pm 0.4$ & $-19.4 \pm 0.4$ & $-18.4 \pm 0.4$ & $2.1 \pm 0.4,1.3 \pm 0.2^{\mathrm{c}}$ & 0.00046 & 0.00043 & 0.00042 \\
\hline So & $-20.5 \pm 0.1$ & $-19.9 \pm 0.1$ & $-19.1 \pm 0.1$ & $1.1 \pm 0.1$ & 0.00130 & 0.00126 & 0.00118 \\
\hline $\mathrm{Sa} / \mathrm{Sb}$ & $-21.2 \pm 0.2$ & $-20.7 \pm 0.2$ & $-19.9 \pm 0.2$ & $0.9 \pm 0.1$ & 0.00699 & 0.00702 & 0.00727 \\
\hline $\mathrm{Sc}$ & $-19.8 \pm 0.2$ & $-19.3 \pm 0.2$ & $-18.7 \pm 0.2$ & $1.2 \pm 0.1$ & 0.00515 & 0.00670 & 0.00800 \\
\hline \multirow[t]{3}{*}{$\mathrm{Sd} / \mathrm{Sm}$} & $-17.7 \pm 0.2$ & $-17.4 \pm 0.2$ & $-17.1 \pm 0.2$ & $0.8 \pm 0.1$ & 0.00417 & 0.00542 & 0.00648 \\
\hline & \multicolumn{3}{|c|}{ Schechter $M^{*}-5 \log h$} & Schechter $\alpha$ & \multicolumn{3}{|c|}{$\phi^{* \mathrm{a}}$} \\
\hline & $R_{\mathrm{c}}{ }^{\mathrm{d}}$ & $V^{\mathrm{d}}$ & $B^{\mathrm{d}}$ & & $R_{\mathrm{c}}$ & V & $B$ \\
\hline $\mathrm{dSph}$ & $-18.9 \pm 0.3$ & $-18.4 \pm 0.3$ & $-17.6 \pm 0.3$ & $-1.7 \pm 0.6 /-1.3 \pm 0.1^{\mathrm{e}}$ & 0.007 & 0.007 & 0.007 \\
\hline $\mathrm{dI}$ & $-17.7 \pm 0.3$ & $-17.4 \pm 0.3$ & $-17.1 \pm 0.3$ & $-1.3 \pm 0.8 /-0.3 \pm 0.2^{\mathrm{e}}$ & 0.04 & 0.05 & 0.06 \\
\hline
\end{tabular}

Table notes:

a The amplitudes $\phi_{0}$ and $\phi^{*}$ are given in $h^{3} \mathrm{Mpc}^{-3} \mathrm{mag}^{-1}$, and are derived from de Lapparent et al. (2003b; see text for details).

b From Sandage et al. (1985) and Jerjen \& Tammann (1997) for E, S0, dSph, and dI galaxies; estimated from Sandage et al. (1985) by de Lapparent et al. (2003b) for $\mathrm{Sa} / \mathrm{Sb}, \mathrm{Sc}, \mathrm{Sd} / \mathrm{Sm}$ galaxies. All values of $M_{0}$ and $M^{*}$ are converted into the $B V R_{\mathrm{c}}$ system by de Lapparent et al. (2003b).

c For E galaxies, the values of $\Sigma_{a}, \Sigma_{b}$ resp. are listed (see Eq. (2)).

d For dSph and dI galaxies, the values of $M^{*}$ estimated from the ESO-Sculptor Survey are listed (de Lapparent et al. 2003b; see text for details).

e For dSph and dI galaxies, the values of $\alpha$ derived from both the Centaurus and Virgo clusters resp. are listed (Jerjen \& Tammann 1997).

skewed towards fainter magnitudes. In contrast, the LFs for dwarf Spheroidal galaxies (denoted dSph) and dwarf Irregular galaxies (denoted dI) are well fit by Schechter functions. The dSph LF has an ever increasing LF at the faint end, whose slope depends on the local galaxy density (Sandage et al. 1985; Ferguson \& Sandage 1991; Pritchet \& van den Bergh 1999; Jerjen et al. 2000; Flint et al. 2001b,a; Conselice et al. 2002) ${ }^{1}$, whereas the dI LF appears to decrease at the faintest magnitudes with a poorly determined shape (Ferguson 1989; Jerjen \& Tammann 1997; Jerjen et al. 2000). Moreover, in all cases examined, the faint end of the LF for the dI galaxies appears to be flatter than for the $\mathrm{dSph}$ galaxies (Pritchet \& van den Bergh 1999).

Table 1 lists the shape parameters measured by Jerjen \& Tammann (1997) for the two-wing Gaussian and pure Gaussian LFs of early-type giant galaxies $(\mathrm{E}, \mathrm{S} 0)$ in the $B_{\mathrm{T}}$ system, and the converted values into $B V R_{\mathrm{c}}$ Johnson-Cousins magnitudes by de Lapparent et al. (2003b) using the results of Schroeder \& Visvanathan (1996) and Fukugita et al. (1995). The Gaussian LF is parameterized as

$$
\phi(M) \mathrm{d} M=\phi_{0} \mathrm{e}^{-\left(M_{0}-M\right)^{2} / 2 \Sigma^{2}} \mathrm{~d} M
$$

where $M_{0}$ and $\Sigma$ are the peak and rms dispersion respectively. Similarly, the two-wing Gaussian is parameterized as

$$
\begin{aligned}
\phi(M) \mathrm{d} M & =\phi_{0} \mathrm{e}^{-\left(M_{0}-M\right)^{2} / 2 \Sigma_{a}^{2}} \mathrm{~d} M \text { for } M \leq M_{0} \\
& =\phi_{0} \mathrm{e}^{-\left(M_{0}-M\right)^{2} / 2 \Sigma_{b}^{2}} \mathrm{~d} M \text { for } M \geq M_{0} .
\end{aligned}
$$

As Jerjen \& Tammann (1997) do not provide the uncertainty in $M_{0}$ for the Elliptical galaxies, I adopt a conservative error

\footnotetext{
${ }^{1}$ Background galaxies may however contaminate the measurements (see Kambas et al. 2000; Valotto et al. 2001; Hilker et al. 2003).
}

of $0.4^{\mathrm{mag}}$ (assuming a similar ratio of the uncertainty in $M_{0}$ by the uncertainty in $\Sigma$ as for the $\mathrm{S} 0$ galaxies). Table 1 also lists the Gaussian parameters for the individual Spiral types $\mathrm{Sa} / \mathrm{Sb}, \mathrm{Sc}$, $\mathrm{Sd} / \mathrm{Sm}$, estimated by de Lapparent et al. (2003b) from Sandage et al. (1985).

Nearby redshift surveys indicate that $\sim 30-40 \%$ of the total number of galaxies in a redshift survey is expected to lie in groups (Ramella et al. 2002), the rest lying in the so-called "field". Ideally, one should therefore compare the intrinsic LFs from redshift surveys to those derived from both field and group of galaxies. Binggeli et al. (1990) do derive LFs for the different types of galaxies in the Ursa Major Cloud (see their Fig. 10), but the statistic is too low to derive usable parameterized LFs from these data. The lack of measurements of the intrinsic LFs for field galaxies with a statistical quality comparable to those for groups/clusters of Sandage et al. (1985) and Jerjen \& Tammann (1997) leads me to refer principally to the latter for defining the shape of the intrinsic LFs listed in Table 1. I nevertheless refer to the field+group LFs derived from the ESO-Sculptor Survey by de Lapparent et al. (2003b) for obtaining estimates of: (i) the characteristic magnitude $M^{*}$ for the dSph and dI LFs; (ii) the amplitudes of the each intrinsic LF listed in Table 1 (see below).

For the dwarf galaxies, the Schechter (1976) parameterization of the LF is

$\phi(M) \mathrm{d} M=0.4 \ln 10 \phi^{*} \mathrm{e}^{-X} X^{\alpha+1} \mathrm{~d} M$

with

$$
X \equiv \frac{L}{L^{*}}=10^{0.4\left(M^{*}-M\right)},
$$


where $M^{*}$ is the characteristic magnitude, and $\alpha$ the "faintend slope". The values of $M^{*}$ for the dSph and dI LFs listed in Table 1 are those estimated from the ESO-Sculptor Survey in the $R_{\mathrm{c}}$ band (de Lapparent et al. 2003b, see their Table 7; I use the average $M^{*}$ for the $R_{\mathrm{c}} \leq 20.5$ and $R_{\mathrm{c}} \leq 21.5 \mathrm{LFs}$, and the comparable uncertainties obtained from the 2 measurements). The values of $M^{*}$ in the $R_{\mathrm{c}}$ band are then converted into the $B$ and $V$ bands using the colors of Sab and Im galaxies: $B-R_{\mathrm{c}}=1.34, V-R_{\mathrm{c}}=0.56 ; B-R_{\mathrm{c}}=0.58$, $V-R_{\mathrm{c}}=0.31$, resp. (Fukugita et al. 1995, see their Table 3a). In the $R_{\mathrm{c}}$ filter, the value of $M^{*}$ for the dSph LF estimated from the ESO-Sculptor Survey (de Lapparent et al. 2003b) is $\sim 0.5^{\mathrm{mag}}$ fainter than in the Virgo cluster (Jerjen \& Tammann 1997), which in turn is $\sim 0.9^{\mathrm{mag}}$ fainter than the value measured in the Centaurus cluster (Jerjen \& Tammann 1997). For the dI galaxies, the value of $M^{*}\left(R_{\mathrm{c}}\right)$ estimated from the ESOSculptor Survey (de Lapparent et al. 2003b) is intermediate between those measured from the Centaurus and Virgo clusters (Jerjen \& Tammann 1997) ${ }^{2}$.

In contrast, I list for the $\mathrm{dSph}$ and $\mathrm{dI}$ LFs in Table 1 the Schechter slope $\alpha$ measured from both the Centaurus and Virgo clusters (Sandage et al. 1985; Jerjen \& Tammann 1997; the steeper slopes correspond to the Centaurus cluster), as these pairs of values describe the range of results obtained for the dSph and dI LFs resp. from the concentrations of galaxies of varying richness; they also include those derived by de Lapparent et al. (2003b) from the ESO-Sculptor Survey. Note that the listed parameters for the dSph and dI LFs where derived by Sandage et al. (1985) and Jerjen \& Tammann (1997) from $\mathrm{dE}+\mathrm{dS}$, and $\mathrm{dI}+\mathrm{BCD}$ (for "Blue Compact Galaxy") respectively; the $\mathrm{dE}$ and $\mathrm{dI}$ galaxies however largely outnumber the dSO and BCD galaxies resp., in both the Virgo and Centaurus clusters.

All values of the LF shape parameters $\left(M_{0}, \Sigma, M^{*}, \alpha\right)$ listed in Table 1 are rounded up/down to the first decimal place. Moreover, the listed uncertainties for the giant galaxy types are those provided for the $B_{\mathrm{T}}$ measurements of the LFs by Sandage et al. (1985) and Jerjen \& Tammann (1997). One should a priori increase the uncertainties when performing the conversion into the $B V R_{\mathrm{c}}$ bands. However, the uncertainties in the LF parameters are only listed here as indicative of the accuracy of the quoted measurements, which frees me from a more detailed treatment.

For graphical comparison of the intrinsic LFs listed in Table 1, one needs to define their respective amplitude. For the dwarf LFs, I adopt and list in Table 1 the average between the values measured from the ESO-Sculptor $R_{\mathrm{c}} \leq 20.5$ and $R_{\mathrm{c}} \leq 21.5$ samples: $\phi^{*}\left(R_{\mathrm{c}}\right)=0.007 h^{3} \mathrm{Mpc}^{-3} \mathrm{mag}^{-1}$ for the $\mathrm{dSph} \mathrm{LF}$ and $\phi^{*}\left(R_{\mathrm{c}}\right)=0.04 h^{3} \mathrm{Mpc}^{-3} \mathrm{mag}^{-1}$ for the dI LF (see Table 7 de Lapparent et al. 2003b); the large uncertainties in these estimates lead us to use only 1 significant digit.

To determine the amplitude $\phi_{0}\left(R_{\mathrm{c}}\right)$ of the Gaussian LFs for the giant galaxy classes listed in Table 1 , I use a combination of constraints derived in the $R_{\mathrm{c}}$ band from the ESO-Sculptor Survey and the Virgo and Centaurus clusters (note that when

\footnotetext{
2 The ESO-Sculptor Survey also excludes the faint value of $M^{*}$ measured from the Virgo cluster (see de Lapparent et al. 2003b).
}

the Virgo and Centaurus LFs provide different constraints, I favor the Centaurus cluster as its lower spatial density better reflects the density of the numerous galaxy groups present in a redshift survey; Ramella et al. 2002). The upper bound of all the integrals mentioned below are obtained by converting the $M\left(B_{\mathrm{T}}\right) \simeq-15.5$ completeness limit from Jerjen \& Tammann (1997) into the $R_{\mathrm{c}}$ band using the quoted colors extracted from Table 5 of de Lapparent et al. (2003b).

For the individual Spiral classes, I use the following constraints, which fully determine the values of $\phi_{0}\left(R_{\mathrm{c}}\right)$ for the $\mathrm{Sa} / \mathrm{Sb}, \mathrm{Sc}$, and $\mathrm{Sd} / \mathrm{Sm} \mathrm{LFs}$ :

- the integral to $M\left(R_{\mathrm{c}}\right) \leq-16.8$ of the intrinsic LF for Sc galaxies is equal to the integral to $M\left(R_{\mathrm{c}}\right) \leq-16.8$ of the intrinsic $\mathrm{LF}$ for the $\mathrm{Sa} / \mathrm{Sb}$ galaxies (the color of an Sbc galaxies is used in both cases), and is twice the integral to $M\left(R_{\mathrm{c}}\right) \leq-16.6$ for the intrinsic $\mathrm{LF}$ for $\mathrm{Sd} / \mathrm{Sm}$ galaxies (the color of an Scd galaxies is used), as suggested by the results from the Centaurus cluster (see Fig. 3 of Jerjen \& Tammann 1997); in the Virgo cluster, the same ratio of the integrals of the $\mathrm{Sc}$ and $\mathrm{Sd} / \mathrm{Sm}$ LFs is observed whereas the ratio of $\mathrm{Sa} / \mathrm{Sb}$ to $\mathrm{Sc}$ galaxies is only $\sim 1 / 2$ (see Fig. 3 of Jerjen \& Tammann 1997). The constraint from the Centaurus cluster provides a relative normalization for the amplitudes $\phi_{0}$ of the $\mathrm{Sa} / \mathrm{Sb}, \mathrm{Sc}$, and $\mathrm{Sd} / \mathrm{Sm} \mathrm{LFs}$;

- the sum of half the LF for $\mathrm{Sa} / \mathrm{Sb}$ galaxies and half the LF for Sc galaxies peaks at $\phi(M)=0.005 h^{3} \mathrm{Mpc}^{-3} \mathrm{mag}^{-1}$, as obtained for the Gaussian component adjusted to the ESO-Sculptor intermediate-type LF (see Table 7 and Fig. 11 of de Lapparent et al. 2003b; I assume that half of the $\mathrm{Sa} / \mathrm{Sb}$ galaxies contribute to each of the ESO-Sculptor early and intermediate-type LFs, and half of the Sc galaxies contribute to each of the ESO-Sculptor intermediate and late-type LFs). Combined with the preceding constraints, this provides the absolute amplitudes $\phi_{0}$ for the $\mathrm{Sa} / \mathrm{Sb}, \mathrm{Sc}$ and $\mathrm{Sd} / \mathrm{Sm} \mathrm{LFs}$.

The amplitudes $\phi_{0}$ of the E and SO LFs are obtained using the following constraints:

- the integral to $M\left(R_{\mathrm{c}}\right) \lesssim-17.15$ of the intrinsic LF for $\mathrm{E}$ galaxies is a factor of 2 smaller than the integral to $M\left(R_{\mathrm{c}}\right) \lesssim-17.15$ of the LF for $\mathrm{S} 0$ galaxies (the average color between those for an E and S0 galaxy is used), which is an acceptable approximation of the results for both the Centaurus and Virgo clusters (see Fig. 3 of Jerjen \& Tammann 1997);

- the sum of the E, S0 and half the $\mathrm{Sa} / \mathrm{Sb}$ LFs peaks at $0.005 h^{3} \mathrm{Mpc}^{-3} \mathrm{mag}^{-1}$, as estimated by the two-wing Gaussian fitted to the ESO-Sculptor early-type LF (see Table 7 and Fig. 11 of de Lapparent et al. 2003b; as already said, I assume that half the $\mathrm{Sa} / \mathrm{Sb}$ galaxies contribute to each of the ESO-Sculptor early and intermediate-type LFs).

The resulting amplitude $\phi_{0}$ in the $R_{\mathrm{c}}$ band for each giant and dwarf galaxy type is listed in Table 1. For all morphological types, I then convert the values of $\phi_{0}\left(R_{\mathrm{c}}\right)$ and $\phi^{*}\left(R_{\mathrm{c}}\right)$ into the $V$ and $B$ bands by multiplying by the ratio of amplitudes $\phi^{*}(V) / \phi^{*}\left(R_{\mathrm{c}}\right)$ and $\phi^{*}(B) / \phi^{*}\left(R_{\mathrm{c}}\right)$ resp., measured 


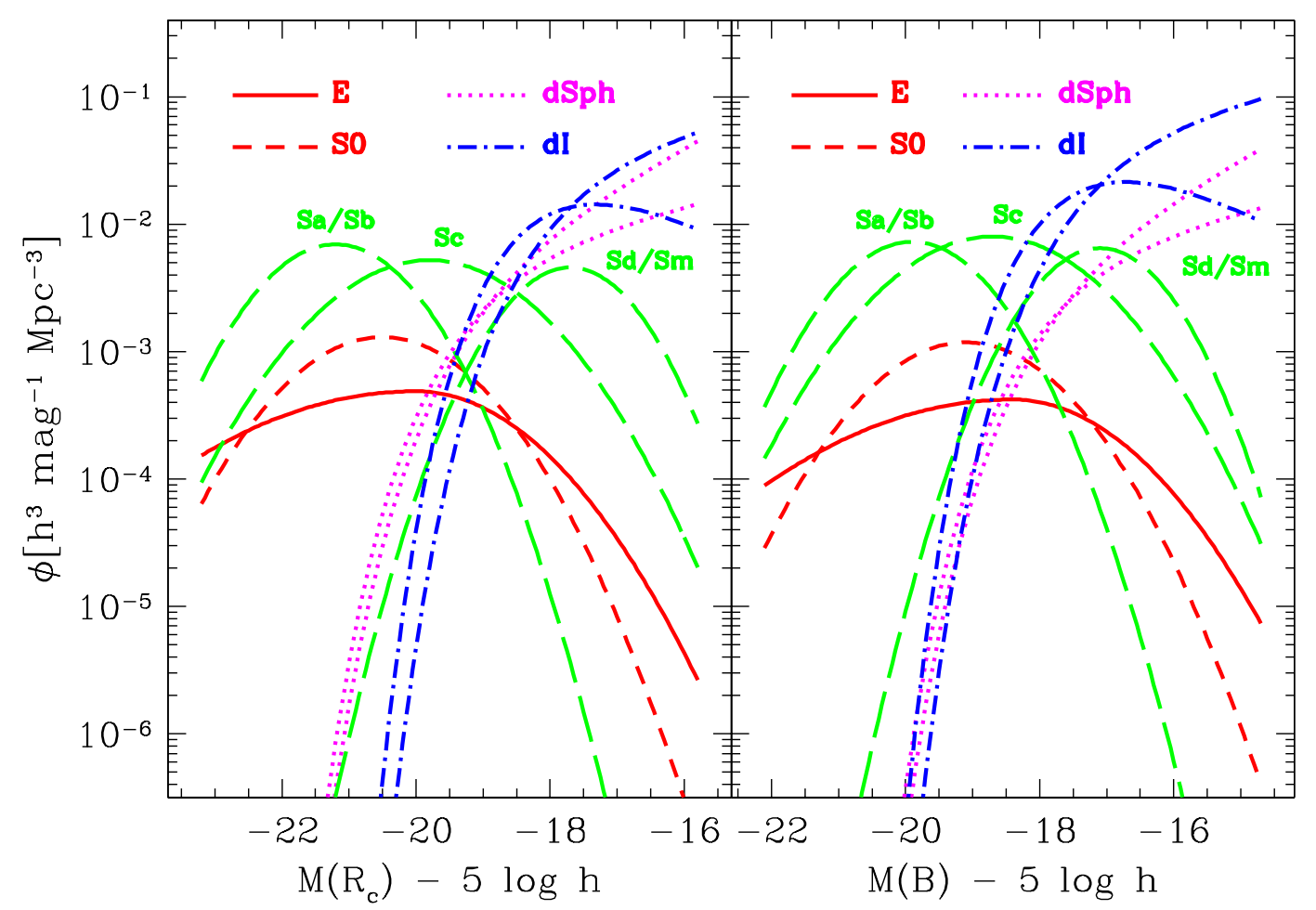

Fig. 1. Intrinsic LFs in the $R_{\mathrm{c}}$ (left panel) and $B$ (right panel) filters with the parameters listed in Table 1 for the morphological types E, S0, $\mathrm{Sa} / \mathrm{Sb}, \mathrm{Sc}, \mathrm{Sd} / \mathrm{Sm}, \mathrm{dSph}$, and dI. The magnitude scale in the $B$ band is shifted by $1.1^{\mathrm{mag}}$ (color of an Sbc galaxy, Fukugita et al. 1995) towards fainter galaxies compared to the $R_{\mathrm{c}}$ band. To describe the range of slopes $\alpha$ measured for the dSph and dI, the 2 LFs with the values of $\alpha$ measured in Virgo and Centaurus resp. are plotted for each type (see Table 1). The amplitudes of the LFs are chosen using the results of Jerjen \& Tammann (1997) and de Lapparent et al. (2003b). This graph shows the relative contribution to the general luminosity function from the various morphological types, as a function of absolute magnitude and filter.

from the Schechter fits to the ESO-Sculptor spectral-type LFs (de Lapparent et al. 2003b, see their Table 3): for the E and S0 LFs listed in Table 1, I use the amplitude ratios for the early-type LFs; for the $\mathrm{Sa} / \mathrm{Sb}$, and $\mathrm{dSph} \mathrm{LFs}$, those for the intermediate-type LFs; for the Sc, Sd/Sm, dI LF, those for the late-type LFs. I emphasize that the resulting values of $\phi_{0}$ and $\phi^{*}$ in the $R_{\mathrm{c}}, V$, and $B$ bands listed in Table 1, are only intended as indicative of the proportions of galaxy types expected in a redshift survey with similar selection effects as in the ESO-Sculptor Survey.

The intrinsic LFs in the $R_{\mathrm{c}}$ and $B$ bands for the parameters listed in Table 1 are plotted in Fig. 1 . The 2 graphs illustrate how each morphological type contributes to the "general" LF in each band. Any measure of LF for a given galaxy sub-sample extracted from a redshift survey is then expected to be some linear combination of the various LFs plotted in Fig. 1, determined by the morphological content of the sample. A wide variety of LF shapes are therefore expected, in agreement with the diversity of results obtained from the surveys described in the following sections.

More specifically, Fig. 1 indicates that in both the $R_{\mathrm{c}}$ and $B$ filters, the bright-end the LF for a redshift survey is systematically dominated by one or several classes among E, SO, Sa/Sb and Sc galaxies, depending of the galaxy types contained in the analyzed sub-sample. For early-type galaxies (based for example on spectral classification or colors), Sc galaxies will poorly contribute to the LF bright-end, whereas for a late-type LF, they might fully determine it. Figure 1 also shows that in both the $R_{\mathrm{c}}$ and $B$ filters, the faint-end of the LF for a sub-sample of galaxies with intermediate spectral type or color, and reaching a lower surface brightness than that for typical giant galaxies, might have its faint-end dominated by dSph galaxies. The faintend of the LF for the bluest or latest-type galaxies is also expected to have a contribution from the dI galaxies, which might dominate over the Spiral galaxy types.

Note that in Fig. 1, the plotted magnitude interval in the $B$ band is shifted by $1.1^{\mathrm{mag}}$ towards fainter galaxies compared to that in the $R_{\mathrm{c}}$ band; this shift corresponds to the color of an Sbc galaxy (see Table 3a of Fukugita et al. 1995). Both panels of Fig. 1 are therefore nearly centered on the Gaussian LF for Sc galaxies. The main differences between the $R_{\mathrm{c}}$ and $B$ band are then caused by both the $B-R_{\mathrm{c}}$ colors of the different galaxy types relative to an Sc galaxy, and the variations in the LF amplitudes with filter. Whereas the galaxy colors are intrinsic (see Fukugita et al. 1995), the LF amplitudes $\phi_{0}$ and $\phi^{*}$ result from the choice which I make of the conversion factors from the $R_{\mathrm{c}}$ into the $B$ band (see above). From the $R_{\mathrm{c}}$ to the $B$ band, the color effects relative to the $\mathrm{Sc} \mathrm{LF}$ are a dimming of the E, S0, Sa/Sb and dSph LFs, and a brightening of the Sd/Sm and dI LFs; the amplitude effects are an increased relative contribution of the $\mathrm{Sc}, \mathrm{Sd} / \mathrm{Sm}$ and dI galaxies compared to the E, S0, Sa/Sb and dSph galaxies. 


\section{Comparison of the luminosity functions from redshift surveys}

All the existing LFs per galaxy class measured from redshift surveys have been fitted by a Schechter (1976) function, characterized by an exponential decrease at bright magnitudes and a power-law behavior at faint magnitudes (see Eq. (3)). When the Schechter parameterization LF is written as a function of absolute magnitude, as in Eq. (3) above, and is viewed in logarithmic coordinates, the faint end has a linear behavior, with a slope $\alpha+1$. The value $\alpha=-1$ is therefore commonly referred to as a "flat slope". As shown in the following Sects., the faint end of the LF in the different surveys describes all possibilities from a steep decrease to a flat or steep increase, which can be modeled by varying values of the Schechter "slope" $\alpha$. Moreover, the value of $M^{*}$ constrains the location of the exponential fall-off of the Schechter function at bright magnitudes. The shapes of the intrinsic LFs can therefore be conveniently compared among them using only the values of $M^{*}$ and $\alpha$ of the Schechter parameterization.

The $M^{*}$ and $\alpha$ parameters for each surveys are listed in Tables 2-4. Figures 2, 4, and 6 provide graphical comparisons of the listed values as a function of filter and redshift interval. For a consistent comparison of the intrinsic LFs for the various surveys, the values of $M^{*}$ in Tables 2-4 and Figs. 2, 4 and 6 have been converted into the $U B V R_{\mathrm{c}} I_{\mathrm{c}}$ Johnson-Cousins system; the color corrections are indicated in the following Sects. This conversion has only been performed for the surveys in which the filter listed in Col. 3 of Tables 2-4 is not among the $U B V R_{\mathrm{c}} I_{\mathrm{c}}$ filters.

For most of the surveys considered here, the values of $M^{*}$ and $\alpha$ were originally derived with $H_{0}=100 h \mathrm{~km} \mathrm{~s}^{-1} \mathrm{Mpc}^{-1}$, $\Omega_{\mathrm{m}}=1.0$, and $\Omega_{\Lambda}=0.0$. The few surveys for which the LFs were only measured for $\Omega_{\mathrm{m}}=0.3$ and $\Omega_{\Lambda}=0.7$ (the surveys denoted CS, COMBO-17 and SDSS-Morph), have been converted into $\Omega_{\mathrm{m}}=1.0$ and $\Omega_{\Lambda}=0.0$ (see Sect. 3.1.1). In the text, when referring to a value of $M^{*}$, I omit the term $+5 \log h$, assumed to be implicit (see Tables 2 to 4). Moreover, the uncertainties in $M^{*}$ and $\alpha$ provided by all authors in the original filters are kept unchanged when converting into the JohnsonCousins system (except for $\alpha[B]$ of the SDSS, see Eq. (4)). For simplicity and because the error ellipses are not provided by the authors, I plot the $\pm 1-\sigma$ error-bars in $M^{*}$ and $\alpha$. There is nevertheless a correlation between the 2 parameters (Schechter 1976) which makes a joint increase or decrease of $M^{*}$ and $\alpha$ less significant than an increase in $M^{*}$ and a decrease in $\alpha$ by the same amount (and vice-versa). When required, the effects of this correlation are taken into account throughout the present analysis.

When provided by the authors, various other parameters defining each sample from which the LFs were calculated are listed in Tables 2 to 4: survey name, solid angle, filter in which the LFs were originally calculated, limiting magnitude of the redshift sample, effective depth or redshift interval used in the calculation of the LF, galaxy class, number of galaxies. The "effective depth" $z_{\max }$ of a survey is defined here as the redshift of an $M^{*}$ galaxy at the apparent magnitude limit of the sample. I however have not calculated this value for each survey, and sometimes only provide an estimate which might differ from the true effective depth by $\$ 20 \%$. When available, the actual redshift interval over which an LF is calculated is listed instead of the effective depth.

In Figs. 2, 4, and 6, the points for a given survey are connected from one class to the next, starting with the earliest class and in order of later type. When the intrinsic LFs are based on galaxy spectral types, the $\left[M^{*}, \alpha\right]$ points for that survey are connected by a solid line. For LFs based on morphological types, the connecting line is dotted. For a criterion based on color or the equivalent width of emission lines, the connecting line is dashed.

\section{1. $U$ and $V$ bands}

The fewest measures of the galaxy intrinsic LFs among the Johnson-Cousins optical bands were obtained in the $U$ and $V$ filters. The values of $M^{*}$ and $\alpha$ in both bands are plotted in Fig. 2 and are listed in Table 2, along with the survey parameters.

\subsubsection{U band}

In the Johnson $U$ band, there is only one survey providing measurements of intrinsic LFs: the CNOC2 (Canadian Network for Observational Cosmology) redshift survey (Lin et al. 1999), plotted in the left panel of Fig. 2. The spectral classification for the CNOC2 galaxies is based on least-square fits of the observed $U B V R_{\mathrm{c}} I \mathrm{c}$ colors to those computed from the galaxy spectral energy distributions (SEDs hereafter) of the templates by Coleman et al. (1980); the 4 types used in these fits are E, Sbc, Scd, and Im, which define "early" (E), "intermediate" (Sbc), and "late" (Scd+Im) spectral types. Although the CNOC2 detects evolutionary effects in $M^{*}$ for the $U$ intrinsic LFs (Lin et al. 1999), I only consider here the $U$ LFs defined by the listed value of $M^{*}$ at $z=0.3$; as no evolution is detected in $\alpha$, I use the unique value provided by the authors. Note that $z=0.3$ corresponds approximately to both the median and peak redshift of the survey (see Fig. 6 of Lin et al. 1999).

Following the general trend detected in most surveys and in all optical bands (see next sections), the faint-end slope $\alpha$ for the CNOC2 $U$ LFs becomes steeper for later type galaxies. However, in contrast to the general dimming of $M^{*}$ for later galaxy types seen in most surveys in the $B V R_{\mathrm{c}}$ bands (see next sections), the values of $M^{*}$ for the intermediate-type and latetype CNOC2 LFs are similar, and are also $\sim 0.7^{\text {mag }}$ brighter than for the early-type LF. Because $-1 \lesssim \alpha \lesssim 0$ for the 3 CNOC2 spectral-type samples, the differences in $M^{*}$ are a good measure of the relative shift in the bright-end of the corresponding LFs. This shift can be explained by the increasing emission in the UV due to star formation in Spiral and Irregular galaxies galaxies (see for example Treyer et al. 1998), making the $U$ magnitude a biased estimate of the total mass of the galaxies.

Recent estimates of UV intrinsic LFs are also provided by the COMBO-17 survey (for "Classifying Objects by 


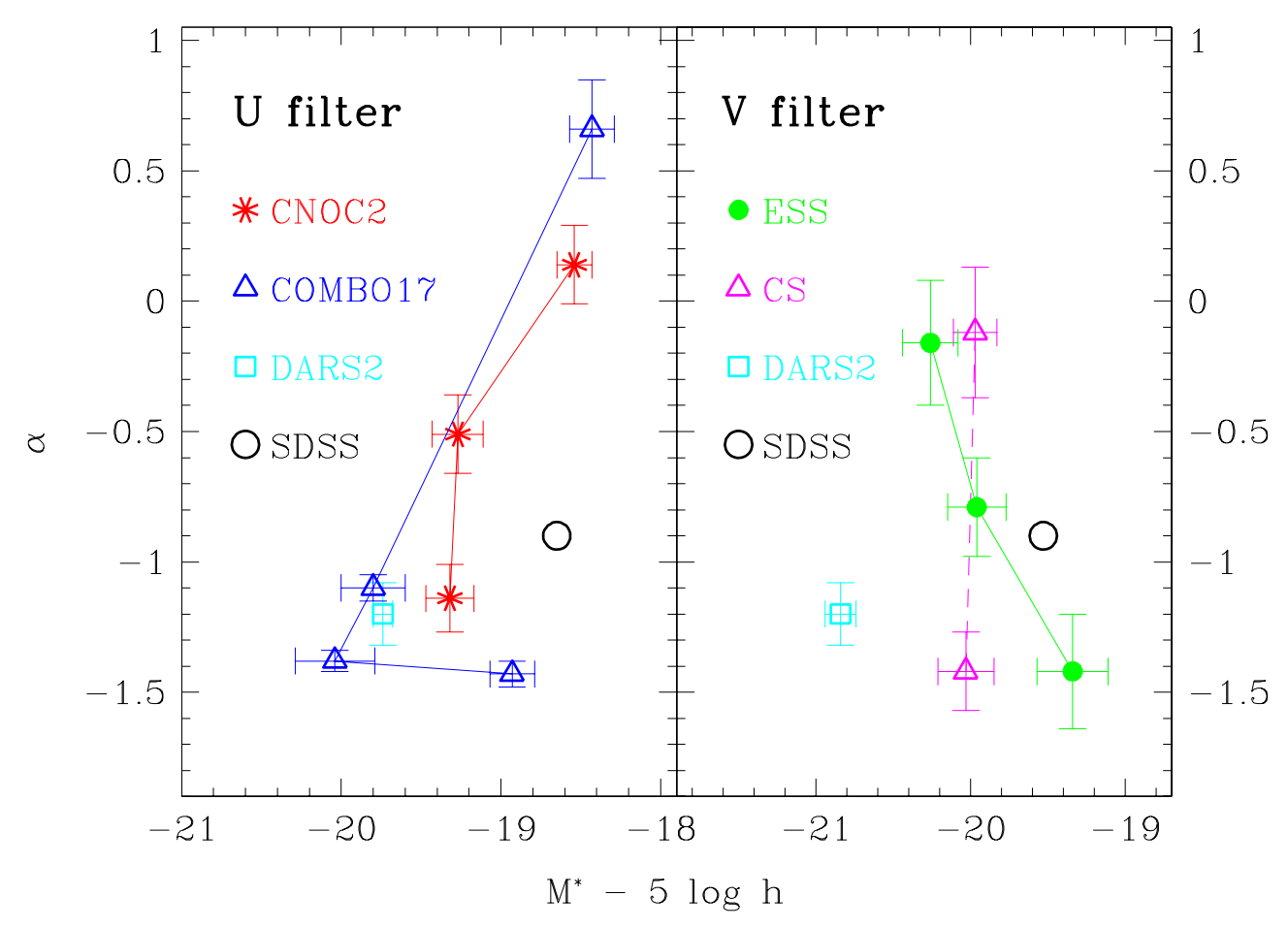

Fig. 2. Comparison of the Schechter parameters $M^{*}$ and $\alpha$ for the existing intrinsic LFs measured or converted into the Johnson $U$ and $V$ bands (see Table 2 for the survey parameters). Other existing surveys providing only a general LF are also indicated (DARS2, SDSS); as the error bars for the SDSS survey are smaller than the symbol size (see Table 2), they are not plotted. Solid, dashed lines connect the various classes of a given survey when these are based on spectral types, or a color cut, respectively. For all surveys, galaxies of later type or with bluer colors are in the direction of steeper slopes $\alpha$ (towards negative values).

Medium-Band Observations in 17 Filters" Wolf et al. 2003), in a synthetic UV continuum band at $\sim 2800 \AA$, denoted $m_{280}$. In contrast to a redshift survey such as the $\mathrm{CNOC} 2$, the spectroscopic catalogue for the COMBO-17 survey is based on a combination of 5 wide-band filters (Johnson $U B V R I)$ and 12 medium-band filters (with $F W H M \simeq 140-310 \AA$ ): spectral types and "photometric" redshifts are obtained by maximizing the summed probability that an observed spectrum matches each template of a given class among the spectral library of Kinney et al. (1996); to this purpose, the authors have replaced the noisy regions of the Kinney et al. (1996) spectra using the PEGASE templates (Fioc \& Rocca-Volmerange 1997). Four spectral classes are then defined: Type-1, Type-2, Type-3 and Type-4, corresponding to Kinney et al. templates E-Sa, Sa-Sbc, Sbc-SB6, and SB6-SB1 resp. (SB is "Starburst", and the number is defined by the value of the $E(B-V)$ color excess, with larger numbers for increasing color excess). The resulting redshift uncertainties are $\sigma(z) \leq 0.03$, to be compared with $\sigma(z) \sim$ 0.0003 for the CNOC2. Although the COMBO-17 LFs are derived for increasing intervals of redshift up to $z \sim 1.2$, here I only examine the intrinsic LFs in the interval $0.2 \lessgtr z \lesssim 0.4$, as it corresponds to the same median redshift $(z \simeq 0.3)$ as in the CNOC2.

The COMBO-17 LFs are only provided for $\Omega_{\mathrm{m}}=0.3$ and $\Omega_{\Lambda}=0.7$. To convert to $\Omega_{\mathrm{m}}=1.0$ and $\Omega_{\Lambda}=0.0$, I define empirical corrections as follows. When changing from $\left[\Omega_{\mathrm{m}}=0.3, \Omega_{\Lambda}=0.7\right]$ to $\left[\Omega_{\mathrm{m}}=1.0, \Omega_{\Lambda}=0.0\right]$, the variation in absolute magnitude due to the change in luminosity distance is $\Delta M \simeq 0.3^{\mathrm{mag}}$ at $z \simeq 0.3$. A change in $M^{*}$ of $0.3^{\mathrm{mag}}$ is therefore expected for the 4 COMBO-17 LFs calculated for $0.2 \lessgtr$ $z \lesssim 0.4$. This empirical correction is confirmed by the results from the CADIS (Fried et al. 2001), based on medium-band photometry as the COMBO-17, and described in Sect. 3.3.1 below: the $B$ LFs for the 3 CADIS spectral types in the interval $0.3<z<0.5$ do show a dimming of $M^{*}$ by $0.3^{\mathrm{mag}}$ from $\left[\Omega_{\mathrm{m}}=0.3, \Omega_{\Lambda}=0.7\right]$ to $\left[\Omega_{\mathrm{m}}=1.0, \Omega_{\Lambda}=0.0\right]$. I also compare the values of the Schechter parameters for the high signalto-noise LFs derived in both Cosmologies from the SDSS (Blanton et al. 2001, see Sect. 3.5). These confirm that when changing from one Cosmology to the other, the dimming in $M^{*}$ is related to the mean variation in luminosity distance over the redshift interval described by each sample (see Tables 1 and 2 from Blanton et al. 2001). Moreover, the $\Delta M$ dimming in $M^{*}$ is accompanied by a flattening in $\alpha$ of $\sim \Delta M / 3$, due to the strong correlation between the 2 Schechter parameters (Blanton et al. 2001). I therefore convert the $U$ COMBO-17 LFs in the interval $0.2<z<0.4$ from $\left[\Omega_{\mathrm{m}}=0.3, \Omega_{\Lambda}=0.7\right]$ to $\left[\Omega_{\mathrm{m}}=1.0\right.$, $\left.\Omega_{\Lambda}=0.0\right]$ by shifting $M^{*}$ and $\alpha$ by $+0.3^{\mathrm{mag}}$ and +0.1 respectively. The COMBO-17 “cosmology-shifted" values of $M^{*}$ in the $m_{280}$ band is then converted into the Johnson $U$ band using the values of $m_{280}-U$ provided by Wolf (2002): $0.92,0.52,-0.09,-0.16$ for the Type-1, Type-2, Type-3, and Type-4 LFs respectively. The resulting LF parameters are listed in Table 2. Note that the same "cosmology-shift" is applied to the COMBO-17 LFs in the $R_{\mathrm{c}}$ and $B$ bands (see Sects. 3.2.1 and 3.3.1). 
Table 2. Schechter parameters for the $U$ and $V$ intrinsic or general LFs measured from the existing redshift surveys.

\begin{tabular}{|c|c|c|c|c|c|c|c|c|c|}
\hline $\begin{array}{l}\text { Survey } \\
\text { (1) }\end{array}$ & $\begin{array}{r}\text { Area } \\
(2)\end{array}$ & $\begin{array}{l}\lambda \\
(3)\end{array}$ & $\begin{array}{l}m_{\lim } \\
(4)\end{array}$ & $\begin{array}{r}z \\
(5)\end{array}$ & $\begin{array}{l}\text { Class } \\
(6)\end{array}$ & $\begin{array}{r}N_{\text {gal }} \\
(7)\end{array}$ & $\begin{array}{c}M^{*}-5 \log h \\
(8)\end{array}$ & $\begin{array}{c}\alpha \\
(9)\end{array}$ & $\begin{array}{c}\text { Comment } \\
\text { (10) }\end{array}$ \\
\hline DARS2 & 70.3 & $U$ & $B \leq 17.0$ & 0.06 & ALL & 288 & $-19.74 \pm 0.06$ & $-1.20 \pm 0.12$ & $\alpha$ fixed from $B \mathrm{LF}$ \\
\hline SDSS & $\sim 2000$ & $u^{*}$ & 18.36 & $0.02-0.14$ & ALL & 22020 & $-18.65 \pm 0.04$ & $-0.90 \pm 0.06$ & \\
\hline \multirow[t]{4}{*}{ COMBO- $17^{\mathrm{a}}$} & 0.78 & $m_{280}$ & $R \lesssim 24.0$ & $0.2-0.4$ & Type-1 & 344 & $-18.43 \pm 0.14$ & $0.66 \pm 0.19$ & fits of obs. SEDs \\
\hline & 0.78 & $m_{280}$ & $R \lessgtr 24.0$ & $0.2-0.4$ & Type-2 & 986 & $-19.80 \pm 0.20$ & $-1.10 \pm 0.05$ & of redshifted temp. \\
\hline & 0.78 & $m_{280}$ & $R \lesssim 24.0$ & $0.2-0.4$ & Type-3 & 1398 & $-20.04 \pm 0.25$ & $-1.38 \pm 0.04$ & \\
\hline & 0.78 & $m_{280}$ & $R \lesssim 24.0$ & $0.2-0.4$ & Type-4 & 2946 & $-18.93 \pm 0.14$ & $-1.43 \pm 0.05$ & \\
\hline \multirow[t]{3}{*}{$\mathrm{CNOC2}$} & 0.692 & $U$ & $R_{\mathrm{c}}<21.5$ & $0.12-0.55$ & Early & 611 & $-18.54 \pm 0.11$ & $0.14 \pm 0.15$ & least-square fit of obs. \\
\hline & 0.692 & $U$ & $R_{\mathrm{c}}<21.5$ & $0.12-0.55$ & Interm & 518 & $-19.27 \pm 0.16$ & $-0.51 \pm 0.15$ & $U B_{\mathrm{AB}} V R_{\mathrm{C}} I_{\mathrm{C}}$ colors \\
\hline & 0.692 & $U$ & $R_{\mathrm{c}}<21.5$ & $0.12-0.55$ & Late & 1017 & $-19.32 \pm 0.15$ & $-1.14 \pm 0.13$ & to redshifted temp. \\
\hline \multirow[t]{2}{*}{$\mathrm{CS}^{\mathrm{a}}$} & 65.3 & $V$ & 16.7 & 0.1 & 1/3-red & 415 & $-19.97 \pm 0.14$ & $-0.12 \pm 0.25$ & $(V-R)_{\text {rest }}>0.551$ \\
\hline & 65.3 & $V$ & 16.7 & 0.1 & 1/3-blue & 424 & $-20.03 \pm 0.18$ & $-1.42 \pm 0.15$ & $(V-R)_{\text {rest }}<0.494$ \\
\hline SDSS & $\sim 2000$ & $g^{*}$ & 17.69 & $0.02-0.17$ & ALL & 53999 & $-19.53 \pm 0.02$ & $-0.91 \pm 0.03$ & \\
\hline \multirow[t]{3}{*}{ ESS } & 0.245 & $V$ & 21.0 & $0.1-0.6$ & Early & 156 & $-20.26 \pm 0.18$ & $-0.16 \pm 0.24$ & PCA-spectral class. \\
\hline & 0.245 & $V$ & 21.0 & $0.1-0.6$ & Interm & 169 & $-19.96 \pm 0.19$ & $-0.79 \pm 0.19$ & \\
\hline & 0.245 & $V$ & 21.0 & $0.1-0.6$ & Late & 168 & $-19.34 \pm 0.23$ & $-1.42 \pm 0.22$ & \\
\hline
\end{tabular}

Table notes:

- Wherever necessary, the listed values of $M^{*}$ result from the conversion from the original values derived by the authors in the filters listed in Col. (3), into the Johnson $U$ and $V$ bands, respectively. The original values of $\alpha$ are kept unchanged.

- All references are provided in the text.

a The values of $M^{*}$ and $\alpha$ for the CS and COMBO-17 surveys are converted from a cosmology with $\left[\Omega_{\mathrm{m}}=0.3, \Omega_{\lambda}=0.7\right]$ into [ $\Omega_{\mathrm{m}}=1.0$, $\left.\Omega_{\lambda}=0.0\right]$ using the empirical corrections described in the text. These values should therefore be used with caution.

Table columns are:

(1) Name of survey.

(2) Survey area in square degrees.

(3) Filter in which the intrinsic or general LFs were originally calculated by the authors.

(4) Limiting magnitude of the photometric sample, in the filter given in the Col. (3) by default, or in some other specified filter.

(5) If one value is given, it is the estimated effective depth $z_{\max }$ of the survey (see text for details), or an upper redshift cut-off. If an interval is given, it is the actual redshift interval used for calculation of the corresponding LF.

(6) Galaxy class defining the sub-sample used for calculation of the corresponding intrinsic LF. When based on morphological types, classes are referred to by the Hubble type. "Early", "interm", "late", "Type-" and "Clan-" refer to spectral types. "ALL" indicates that the "general" LF is listed; this is used for the samples for which no intrinsic LFs are provided: SDSS, DARS2, and DUKST (see Sect. 3.5). The scheme for galaxy classification is specified in the last column labeled "Comment".

(7) Number of galaxies in the sample/sub-sample used for calculation of the LF.

(8) Characteristic magnitude of the LF Schechter parameterization for the sample/sub-sample.

(9) Slope at faint magnitudes of the LF Schechter parameterization for the sample/sub-sample.

(10) Comment on the sample/sub-sample, plus specification of the scheme used for classifying galaxies: if too long, the description of the classification scheme is written over several rows of the table; it however applies to all classes of the considered survey.

The Johnson $U$ LFs derived from the COMBO-17 survey show similarities and differences with the CNOC2 $U$ LFs. Despite the "cosmology-shift" and the large $m_{280}-U$ color correction for the COMBO-17 Type-1 galaxies (see above), their value of $M^{*}$ is consistent with that for the early-type CNOC2 galaxies at less than the $1-\sigma$ level (the variance in the difference between 2 measures of $M^{*}$ or $\alpha$ is estimated as the quadrature sum of the uncertainties in the 2 measures). In contrast, the COMBO-17 value of $\alpha$ departs from the CNOC2 value by 2.1- $\sigma$. This difference in the value of $\alpha$ is consistent with the small fraction of galaxies included in the COMBO-17 Type-1 class (corresponding to Kinney et al. 1996 spectral types E-Sa): they represent only $6 \%$ of the galaxies with $0.2<z<0.4$, whereas the early-type classes in the $\mathrm{CNOC} 2$ (corresponding to Coleman et al. 1980 spectral type E) contains $29 \%$ of the galaxies. Although the Type- 1 galaxies in the COMBO-17 are selected using E-Sa template spectra, this class is dominated by E and S0 (see also Fig. 2 from Wolf et al. 2003), whereas the CNOC2 early-type classes contain in addition a significant number of $\mathrm{Sa}$ and $\mathrm{Sab}$ galaxies. As shown by de Lapparent et al. (2003b), dSph galaxies which might cause the flat faint-end slope of the ESO-Sculptor intermediate class 
can have optical colors comparable to those for Sab galaxies (see also Sect. 2); these objects might therefore also contaminate the CNOC2 early-type LF, while being excluded from the COMBO-17 Type-1 class, which could in turn explain the "flatter" value of $\alpha$ for the CNOC2 early-type LF.

The $U$ LFs for both the COMBO-17 and CNOC2 show a brightening of $M^{*}$ from the early to intermediate spectral classes. This brightening is however larger for the COMBO-17, with a $1.4^{\mathrm{mag}}$ brightening of $M^{*}$ between the Type- 1 and the Type-2 LFs; it corresponds to a similar shift of the LF brightend (see Figs. A.11 and A.12 in Wolf et al. 2003), to be compared to the $\sim 0.5^{\mathrm{mag}}$ brightening in the value of $M^{*}$ between the CNOC2 early-type LF and both the intermediate and late-type LFs. The COMBO-17 Type-3 class (Kinney et al. 1996 spectral types Sbc-SB6) and the CNOC2 intermediatetype class (Coleman et al. 1980 spectral type Sbc) are expected to have a significant number of galaxies in common, due to their similar spectral content. The COMBO-17 Type-3 LF however has a steeper $\alpha$ at the 5.8- $\sigma$ level, and a brighter $M^{*}$ at the 2.6- $\sigma$ level compared to the CNOC2 intermediate-type LF. The COMBO-17 Type-2 LF (spectral types Sa-Sbc) also has a steeper $\alpha$ at the 3.7- $\sigma$ level and brighter $M^{*}$ at the 2.1- $\sigma$ level compared to the CNOC2 intermediate-type LF, whereas one would expect the Type-2 LF to be intermediate between the CNOC2 early-type (spectral types E) and intermediate-type (spectral type $\mathrm{Sbc}$ ). Note that the elongation of the error ellipses for the Schechter parameterization in the direction of brighter $M^{*}$ and steeper $\alpha$ would actually decrease the quoted significance levels. These would however remain larger than 2- $\sigma$ for the difference in $\alpha$.

One explanation could be related to the uncertainties in the absolute magnitudes induced by the $\sigma(z) \sim 0.03$ redshift errors in the COMBO-17. For faint Starburst galaxies, the redshifts errors are even larger, $\sigma(z) \sim 0.1$, and imply magnitude errors of 0.75 mag. Wolf et al. (2003, see the end of their Sect. 3.5) warn that these uncertainties tend to "bias the steep luminosity function of Starburst galaxies to brighter $L^{*}$ values". A significant contamination of the Type-2 (spectral types Sa-Sbc) and Type-3 (spectral types Sbc-SB6) classes by Starburst galaxies, despite the small expected number of such objects in these classes, could explain the bright $M^{*}$ for the corresponding LFs. Surprisingly, this luminosity bias does not appear to affect the Type-4 LF (spectral types SB6-SB1) which only differs from the CNOC2 late-type LF (spectral types Scd/Im) at the $\sim 2-\sigma$ level in both $M^{*}$ and $\alpha$, with a fainter $M^{*}$ and a steeper $\alpha$; this is in agreement with the similar fractions of galaxies in the COMBO-17 Type-4 and the CNOC2 late-type samples (52\% and $47 \%$ resp.). The Type- 4 class is however supposed to contain only Starburst galaxies, for which the luminosity bias is expected to be the largest. Other complex selection effects inherent to surveys based on multi-medium-band photometry, and most critical for emission-line galaxies, might also be at play in the COMBO-17. Another possible interpretation is that the LFs for the COMBO-17 Spiral galaxies ( $\mathrm{Sa}, \mathrm{Sb}, \mathrm{Sc}$ ) which dominate the Type-2 and Type-3 class and may have significant emission-lines, may also be biased towards bright values of $M^{*}$, whereas the Type-4 class succeeds in separating the lowermass Irregular galaxies populating the LF (see Fig. 1). The absence of systematic brightening of $M^{*}$ for the COMBO-17 Type-4 LF compared to the CNOC2 late-type LF could then result from the combination of a systematic brightening affecting the Type-4 galaxies which would be compensated for by an intrinsic fainter $M^{*}$ than in the CNOC2 late-type LF. At last, the COMBO-17 color transformations from the synthetic UV continuum band $m_{280}$ into the Johnson $U$ band (Wolf 2002) might suffer some biases, possibly related to the large difference $(\sim 1000 \AA)$ between the peaks in the response curves of the respective filters.

\subsection{2. $V$ band}

There are so far only 2 estimates of intrinsic LFs in the Johnson $V$ band: the Century Survey (Brown et al. 2001, denoted CS), for which the LFs are calculated from the $1 / 3$ blue and $1 / 3$ red portions of the full sample, based on $V-$ $R_{\mathrm{c}}$ rest-frame color (see Table 2 for the color bounds); and the ESO-Sculptor survey (de Lapparent et al. 2003b, denoted ESS), which provides the first measurements of $V$ intrinsic LFs based on 3 spectral classes. LF measurements from both surveys are plotted in the right panel of Fig. 2. Note that the ESS only detects evolution in the amplitude of the late-type LF (de Lapparent et al. 2003a); the listed values of $M^{*}$ and $\alpha$ in Table 2 are those derived from the full redshift range of the ESS. As the intrinsic LFs for the CS 1/3-red and 1/3-blue sub-samples are only provided for cosmological parameters $\Omega_{\mathrm{m}}=0.3$ and $\Omega_{\lambda}=0.7$, I convert the $M^{*}, \alpha$ values provided by the authors to $\Omega_{\mathrm{m}}=1.0$ and $\Omega_{\Lambda}=0.0$ by adding 0.1 and 0.03 resp., based on the variations for the full $V$ sample (see Table 2 of Brown et al. 2001); note that these offsets in $M^{*}$ and $\alpha$ are consistent with those which would be inferred by the empirical method which I use above for converting the COMBO-17 LFs to $\left[\Omega_{\mathrm{m}}=1.0, \Omega_{\Lambda}=0.0\right]$, and which is based on the mean variation of the luminosity distance over the considered sample, when changing the cosmological parameters.

The faint-end slope $\alpha$ for both the $1 / 3$ red and $1 / 3$ blue LFs in the Century Survey are in agreement with those for the ESS early-type and late-type LFs, respectively. In contrast, the values of $M^{*}(V)$ for the Century Survey LFs are nearly equal; Figs. 15 and 16 of Brown et al. (2001) however show that the bright ends of the 2 LFs differ by $0.5^{\mathrm{mag}}$. A similar effect is present in the ESS LFs: there is a $\sim 0.9^{\text {mag }} \mathrm{dim}$ ming of $M^{*}(V)$ between the early-type and the late-type LFs, whereas the bright-end of the early-type and late-type LFs are shifted by a larger amount, $\sim 1.5^{\mathrm{mag}}$. The strong correlation between $M^{*}$ and $\alpha$ in the Schechter parameterization implies that the difference in the value of $M^{*}$ for 2 different LFs may not be a measure of the actual shift in the bright-end fall-off for these 2 LFs. An exact correspondence only occurs if the 2 LFs have the same value of $\alpha$. The various surveys examined in the present article show that for $-1 \lesssim \alpha \lesssim 0$, the agreement is within $0.1 \mathrm{mag}$. For steeper values of $\alpha$, in the interval $-2 \lesssim \alpha \lesssim-1$, a shift $\Delta M$ in the LF bright-end corresponds to a change in $M^{*}$ by $\Delta M^{*} \simeq \Delta M-f(\alpha)$, with $0.5 \lesssim f(\alpha) \lesssim 1^{\mathrm{mag}}$. 


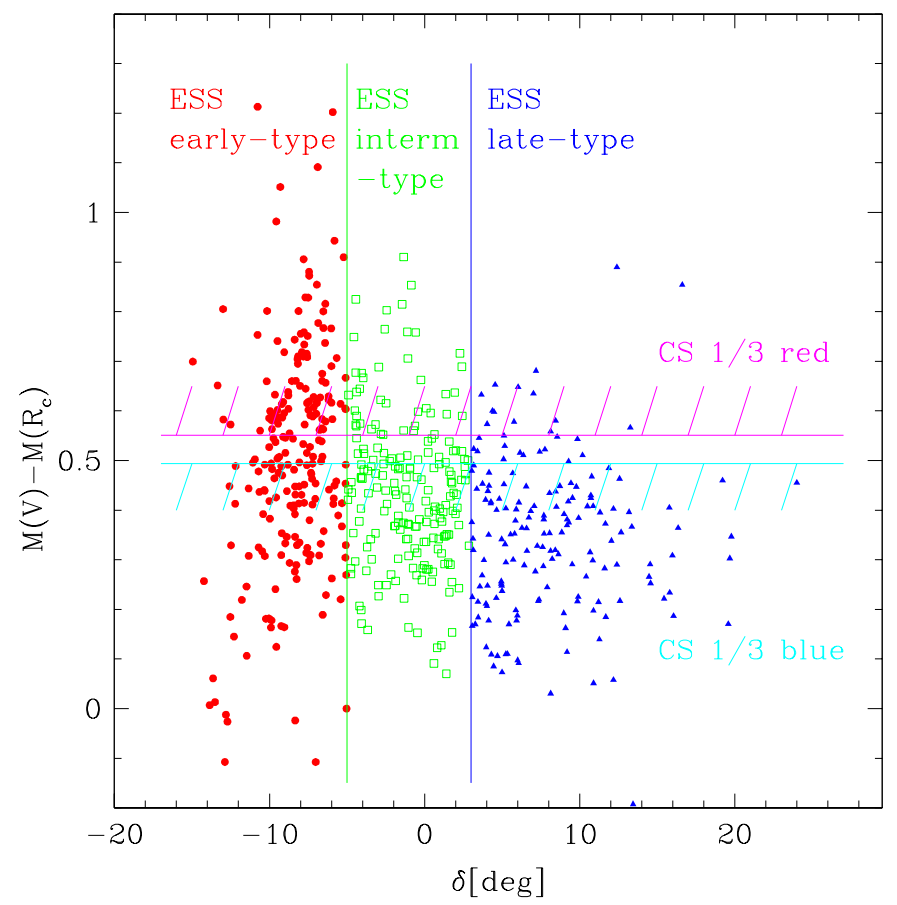

Fig. 3. Relation between the ESO-Sculptor (ESS) PCA spectral type $\delta$ and the absolute color $M(V)-M\left(R_{\mathrm{c}}\right)$ of each galaxy. The ESOSculptor early, intermediate and late-type galaxies are defined by the intervals $\delta \leq-5.0^{\circ},-5.0<\delta \leq 3.0^{\circ}$, and $\delta>3.0^{\circ}$ resp., separated by the 2 vertical lines. The color cuts used for the measurement of the Century Survey (CS) LFs are superimposed as the 2 horizontal lines: the Century Survey $1 / 3$ blue and $1 / 3$ red sub-samples are defined by $M(V)-M\left(R_{\mathrm{c}}\right)<0.494$ and $M(V)-M\left(R_{\mathrm{c}}\right)>0.551$ respectively. This graph shows how sub-samples based on a color cut mix galaxies of different spectral types.

In the ESS, the dimming of $M^{*}(V)$ for later spectral types confirms that $M(V)$ is a better estimate of the total mass of the galaxies than $M(U)$. This dimming is interpreted by de Lapparent et al. (2003b) as a signature of the fainter magnitude late-type Spiral galaxies (Sc, Sd, Sm) detected in the late-type class, compared to the brighter earlier type Spiral galaxies $\mathrm{Sa}$ and $\mathrm{Sb}$ included in the early and intermediate-type classes resp. (see also Sect. 2 and Fig. 1). The smaller dimming in $M^{*}(V)$ for the CS compared to the ESS can be explained as a result of the color cut for separating the LF sub-samples, which causes some mixing of the spectral types. Figure 3 illustrates this effect by showing the distribution of ESS spectral types $\delta$, as a function of absolute (or rest-frame) color $M(V)-$ $M\left(R_{\mathrm{c}}\right)$ for each galaxy. In the ESS, the spectral type $\delta$ is obtained by a classification based on a Principal Component Analysis (PCA hereafter), and is tightly correlated with the morphological type (de Lapparent et al. 2003b); the earlytype, intermediate-type, and late-type classes, are defined by $\delta \leq-5.0^{\circ},-5.0<\delta \leq 3.0^{\circ}$, and $\delta>3.0^{\circ}$ resp. (shown as vertical lines in Fig. 3), and contain predominantly E/S0/Sa, Sb/Sc, and $\mathrm{Sc} / \mathrm{Sm} / \mathrm{Im}$ galaxies resp. (de Lapparent et al. 2003b).

By applying to the ESS the colors cuts used in the CS for defining the $1 / 3$ red and $1 / 3$ blue sample, Fig. 3 shows that the 2 color samples contain significant fractions of galaxies from several spectral classes: the blue sample contains $27.3 \%, 35.2 \%$, and $37.5 \%$ of early-type, intermediatetype, and late-type galaxies resp., and the red sample, $62.5 \%$, $26.9 \%$, and $10.6 \%$ respectively. Therefore, the red sample is dominated by the early-type galaxies and to a smaller extent, by the intermediate-type galaxies; in contrast, the blue sample contains comparable fractions of the 3 galaxy spectral types. The steeper slope for the CS $1 / 3$ blue LF compared to the $1 / 3$ red LF reflects the fact that the majority of the galaxies of late spectral type are included in the $1 / 3$ blue sample (see Fig. 3). The 2 LFs however have comparable $M^{*}$ because its determination is dominated by the brightest galaxies in the 2 color samples, namely the E/S0/Sa/Sb galaxies which populate the early-type and intermediate-type spectral classes, both included in the 2 color samples. This analysis illustrates how intrinsic LFs based on 2 color classes fail to separate the blue low luminosity galaxies from the more luminous Elliptical and Spiral galaxies.

Note that the fainter peak surface brightness limit reached in the ESS $\left(\simeq 22-22.5 \mathrm{~V}\right.$ mag $\operatorname{arcsec}^{-2}$, de Lapparent et al. 2003b), compared to $\simeq 20-21 V$ mag $\operatorname{arcsec}^{-2}$ in the CS (Brown et al. 2001) $)^{3}$, might also contribute to a better detection of Irregular galaxies which dominate the ESS late spectral-types at faint magnitudes $\left(M\left(R_{\mathrm{c}}\right) \gtrsim-18.5\right.$; see Fig. 1) and have lower surface brightness than Elliptical and Spiral galaxies.

\section{2. $R_{\mathrm{c}}$ band}

The existing measurements of intrinsic LFs in the $R_{\mathrm{c}}$ band are more numerous than in the $U$ and $V$ bands. They are listed in Table 3 and plotted in Fig. 4.

\subsection{1. $R_{\mathrm{C}}$ luminosity functions at redshifts $0.4-0.6$}

The right panel of Fig. 4 displays the estimates of intrinsic LF from the 4 surveys with $0.4 \lesssim z_{\max } \lesssim 0.6$ : the CNOC2 survey (Lin et al. 1999), already mentioned in Sect. 3.1.1, and its cluster analog, the CNOC1 survey, which also provides a sample of field galaxies (Lin et al. 1997); the ESS (de Lapparent et al. 2003b), and the COMBO-17 (Wolf et al. 2003), both already mentioned in Sects. 3.1.2 and 3.1.1 respectively. For the CNOC1, survey, the Thuan \& Gunn (1976) $r$ magnitudes are converted into $R_{\mathrm{c}}$ by applying the conversion $R_{\mathrm{c}}=r-0.36$, as calculated by Fukugita et al. (1995) for an Sbc galaxy; no distinction is made for the various spectral types as $r-R_{\mathrm{c}}$ varies in the narrow interval $0.34-0.38$ among the 6 galaxy types listed by Fukugita et al. (1995). Because the COMBO-17 red LFs are measured in the Sloan Digital Sky Survey $r^{*}$ band (Fukugita et al. 1996), I convert the "cosmology-corrected" values of $M^{*}\left(r^{*}\right)$ (see Sect. 3.1.1) into the Johnson $R$ band using the values of $r^{*}-R$ provided by Wolf (2002): $0.3,0.3,0.22,0.16$ for Type-1, Type-2, Type-3, and Type-4 galaxies respectively. I then apply the $R_{\mathrm{c}}-R$ colors terms provided by Fukugita et al. (1995, see their Table 3) for types S0, Sbc, Scd, and Im: 0.09, $0.08,0.07$, and 0.03 respectively.

\footnotetext{
${ }^{3}$ In both surveys, the surface brightness is corrected for the $2.5 \log (1+z)^{4}$ redshift-dimming; in the ESS, K-corrections are also applied (see de Lapparent et al. 2003b).
} 


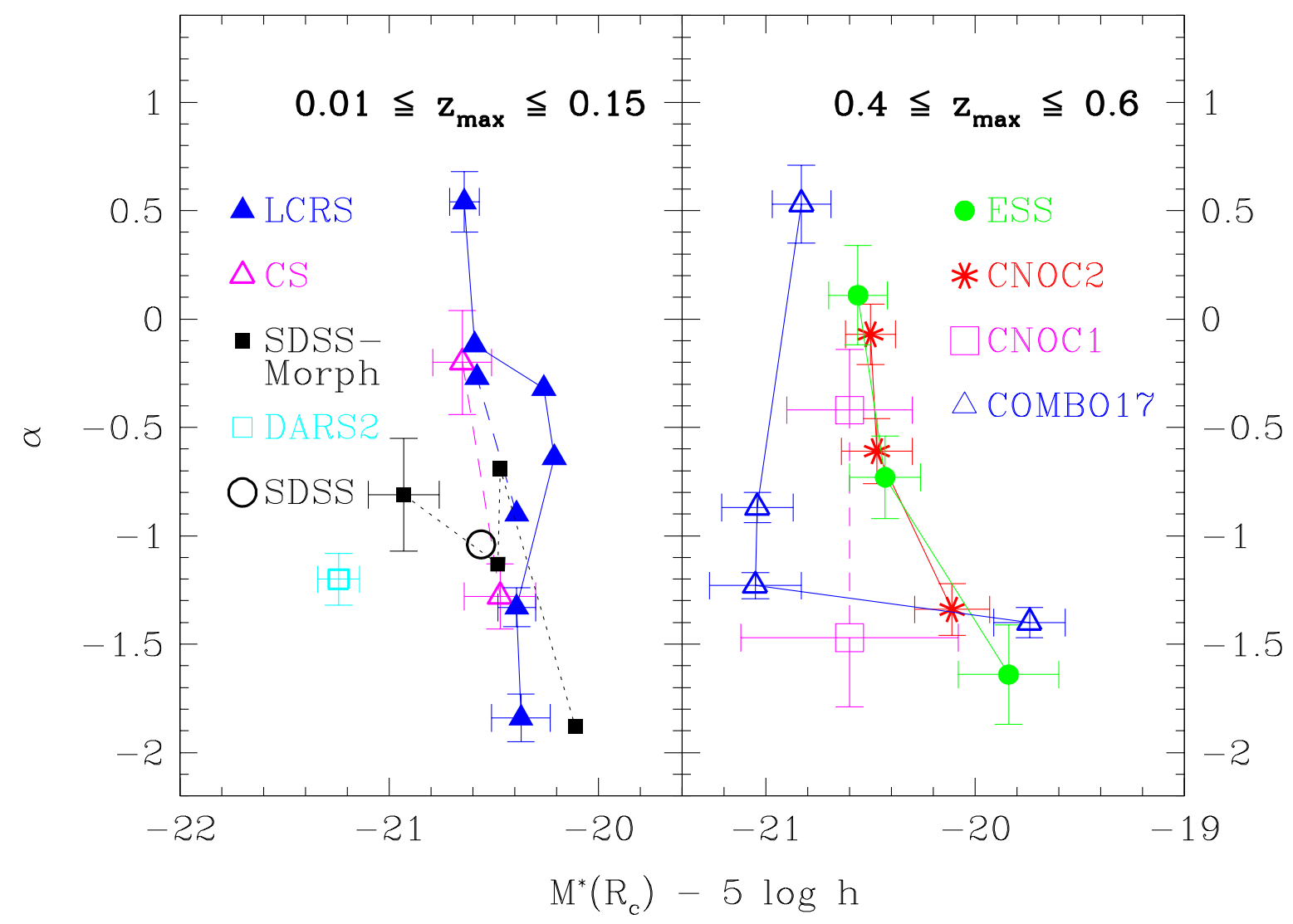

Fig. 4. Comparison of the Schechter parameters $M^{*}$ and $\alpha$ for the existing intrinsic LFs measured or converted into the Cousins $R_{\mathrm{c}}$ band. The surveys with effective depth $0.01 \leq z_{\max } \leq 0.15$ are shown in the left panel, those with $0.4 \leq z_{\max } \leq 0.6$ in the right panel (see Table 3 for the survey parameters). The general LFs provided by the DARS2 and SDSS are also shown (see Sect. 3.5; the error bars for the SDSS are smaller than the symbol size). The error bars are not shown for several points of the LCRS survey for which it is nearly equal or smaller than the symbol size (see Table 3). For clarity, the error bars for the morphological LFs of the SDSS are only shown for the E-S0 LF; the error bars for types S0/Sa-Sb Sbc-Sd are similar, whereas no error bar are provided for Type Im by Nakamura et al. (2003). Solid, dashed, and dotted lines connect the various classes of a given survey when these are based on spectral types, a color cut or the equivalent width of emission lines, and morphological types respectively. For all surveys, galaxies of later type, with bluer colors or stronger emission lines are in the direction of steeper slopes $\alpha$ (towards negative values).

Because the CNOC2, COMBO-17 and ESS all detect evolutionary effects in some of their $R_{\mathrm{c}}$ intrinsic LFs, I restrict the comparison to the LFs measured at the median and/or peak redshift of $z \simeq 0.3$. As in the $U$ band, I use for the CNOC2 the listed values of the evolving $M^{*}$ at $z=0.3$, and the nonevolving value of $\alpha$ (Lin et al. 1999). For the COMBO-17 survey, I use the intrinsic LFs in the interval $0.2 \lesssim z \lesssim 0.4$. For the ESS, the mean LFs over the full redshift range of the survey are provided by de Lapparent et al. (2003b).

The measured values of $M^{*}\left(R_{\mathrm{c}}\right)$ and $\alpha$ for the CNOC2 and ESS are in good agreement. de Lapparent et al. (2003b) show that the ESS $R_{\mathrm{c}}$ early-type LF is consistent with a Gaussian parameterization, in agreement with the Gaussian LFs measured locally for E, S0, and Sa galaxies (see also Fig. 1 in Sect. 2 above). The similar $M^{*}$ and $\alpha$ parameters for the CNOC2 $R_{\mathrm{c}} \mathrm{LF}$ indicate that a Gaussian parameterization might also be appropriate for the early-type LF for that sample. Both samples show a steepening of $\alpha$ when going to later spectral types, and a dimming of $M^{*}(R)$ by $\sim 0.6^{\mathrm{mag}}$ when going from intermediatetype to late-type galaxies, with most of the dimming occurring between the intermediate and late-type LFs. As in the $V$ band, this dimming is due to the fainter galaxies (types $\mathrm{Sc} / \mathrm{Sm} / \mathrm{Im}$ ) included in the ESS and CNOC2 late-type classes (see Fig. 1).

The general agreement of the ESS and $\mathrm{CNOC} 2$ intrinsic LFs in the $R_{\mathrm{c}}$ band is a result of the similar morphological content of the spectral classes, as shown by de Lapparent et al. (2003b): the early, intermediate, and late-type classes contain predominantly E/S0/Sa, Sb/Sc, and Sc/Sm/Im resp. in the ESS, and $\mathrm{E} / \mathrm{Sab}, \mathrm{Sbc}$, and $\mathrm{Scd} / \mathrm{Im}$ resp. in the $\mathrm{CNOC2}$; when extrapolated to $R_{\mathrm{c}} \leq 21.5$, the ESS early, intermediate, and late-type classes contain $27 \%, 30 \%$, and $43 \%$ resp. of the total number of galaxies, and the CNOC2 classes contain $29 \%, 24 \%$, and $47 \%$ respectively. Given the 1-mag difference in the magnitude limit for the 2 surveys, the differing selection effects and redshift completeness curves, this agreement is remarkable. The slight shift toward galaxies of earlier type in the CNOC2 late-type class, as indicated by the fractions of galaxies per spectral type, might also explain why this LF has a flatter $\alpha$ and brighter $M^{*}$ than in the ESS. 
Table 3. Schechter parameters for the $R_{\mathrm{c}}$ and $I_{\mathrm{c}}$ intrinsic or general LFs measured from the existing redshift surveys.

\begin{tabular}{|c|c|c|c|c|c|c|c|c|c|}
\hline $\begin{array}{l}\text { Survey } \\
\text { (1) }\end{array}$ & $\begin{array}{r}\text { Area } \\
(2)\end{array}$ & $\begin{array}{l}\lambda \\
(3)\end{array}$ & $\begin{array}{l}m_{\lim } \\
(4)\end{array}$ & $\begin{array}{l}z \\
(5)\end{array}$ & $\begin{array}{l}\text { Class } \\
(6)\end{array}$ & $\begin{array}{r}N_{\text {gal }} \\
(7)\end{array}$ & $\begin{array}{c}M^{*}-5 \log h \\
(8)\end{array}$ & $\begin{array}{c}\alpha \\
(9)\end{array}$ & $\begin{array}{c}\text { Comment } \\
\text { (10) }\end{array}$ \\
\hline \multirow[t]{2}{*}{$\mathrm{CS}^{\mathrm{a}}$} & 65.3 & $R_{\mathrm{c}}$ & 16.2 & 0.1 & $1 / 3$-red & 419 & $-20.65 \pm 0.14$ & $-0.20 \pm 0.24$ & $(V-R)_{\text {rest }}>0.555$ \\
\hline & 65.3 & $R_{\mathrm{c}}$ & 16.2 & 0.1 & 1/3-blue & 422 & $-20.47 \pm 0.17$ & $-1.28 \pm 0.15$ & $(V-R)_{\text {rest }}<0.499$ \\
\hline DARS2 & 70.3 & $R_{\mathrm{c}}$ & $B \leq 17.0$ & 0.06 & ALL & 288 & $-21.24 \pm 0.10$ & $-1.20 \pm 0.12$ & alpha fixed from $B \mathrm{LF}$ \\
\hline \multirow[t]{4}{*}{ SDSS-Morph } & 230.0 & $r^{*}$ & 15.90 & $0.01-0.075$ & $\mathrm{E} / \mathrm{S} 0$ & 597 & $-20.93 \pm 0.17$ & $-0.81 \pm 0.26$ & $0 \leq T \leq 1.0$ \\
\hline & 230.0 & $r^{*}$ & 15.90 & $0.01-0.075$ & $\mathrm{~S} 0 / \mathrm{Sa} / \mathrm{Sb}$ & 518 & $-20.48 \pm 0.19$ & $-1.13 \pm 0.26$ & $1.5 \leq T \leq 3$ \\
\hline & 230.0 & $r^{*}$ & 15.90 & $0.01-0.075$ & $\mathrm{Sbc} / \mathrm{Sd}$ & 350 & $-20.47 \pm 0.20$ & $-0.69 \pm 0.26$ & $3.5 \leq T \leq 5$ \\
\hline & 230.0 & $r^{*}$ & 15.90 & $0.01-0.075$ & $\mathrm{Im}$ & 10 & -20.11 & -1.88 & $5.5 \leq T \leq 6$ \\
\hline SDSS & $\sim 2000$ & $r^{*}$ & 17.79 & $0.02-0.22$ & ALL & 147986 & $-20.56 \pm 0.03$ & $-1.04 \pm 0.03$ & \\
\hline \multirow[t]{8}{*}{ LCRS } & 462 & $r$ & 17.7 & 0.15 & OII & 7312 & $-20.03 \pm 0.03$ & $-0.90 \pm 0.04$ & $E W[\mathrm{OIII}]>5 \AA$ \\
\hline & 462 & $r$ & 17.7 & 0.15 & no-OII & 11366 & $-20.22 \pm 0.02$ & $-0.27 \pm 0.04$ & $E W[\mathrm{OIII}]<5 \AA$ \\
\hline & 462 & $r$ & 17.7 & 0.15 & Clan-1 & 655 & $-20.64 \pm 0.07$ & $0.54 \pm 0.14$ & PCA-spectral class. \\
\hline & 462 & $r$ & 17.7 & 0.15 & Clan-2 & 7614 & $-20.59 \pm 0.03$ & $-0.12 \pm 0.05$ & \\
\hline & 462 & $r$ & 17.7 & 0.15 & Clan-3 & 4667 & $-19.26 \pm 0.04$ & $-0.32 \pm 0.07$ & \\
\hline & 462 & $r$ & 17.7 & 0.15 & Clan-4 & 3210 & $-19.21 \pm 0.05$ & $-0.64 \pm 0.08$ & \\
\hline & 462 & $r$ & 17.7 & 0.15 & Clan-5 & 1443 & $-20.39 \pm 0.09$ & $-1.33 \pm 0.09$ & \\
\hline & 462 & $r$ & 17.7 & 0.15 & Clan-6 & 689 & $-20.37 \pm 0.14$ & $-1.84 \pm 0.11$ & \\
\hline \multirow[t]{4}{*}{ COMBO- $17^{\mathrm{a}}$} & 0.78 & $r^{*}$ & $R \lesssim 24.0$ & $0.2-0.4$ & Type-1 & 344 & $-20.83 \pm 0.14$ & $0.53 \pm 0.18$ & fits of obs. SEDs \\
\hline & 0.78 & $r^{*}$ & $R \lesssim 24.0$ & $0.2-0.4$ & Type-2 & 986 & $-21.04 \pm 0.17$ & $-0.87 \pm 0.07$ & of redshifted temp. \\
\hline & 0.78 & $r^{*}$ & $R \lesssim 24.0$ & $0.2-0.4$ & Type-3 & 1398 & $-21.05 \pm 0.22$ & $-1.23 \pm 0.06$ & \\
\hline & 0.78 & $r^{*}$ & $R \lesssim 24.0$ & $0.2-0.4$ & Type-4 & 2946 & $-19.74 \pm 0.17$ & $-1.40 \pm 0.07$ & \\
\hline \multirow[t]{3}{*}{$\mathrm{CNOC} 2$} & 0.692 & $R_{\mathrm{c}}$ & 21.5 & 0.55 & Early & 611 & $-20.50 \pm 0.12$ & $-0.07 \pm 0.14$ & least-square fit of obs. \\
\hline & 0.692 & $R_{\mathrm{c}}$ & 21.5 & 0.55 & Interm & 517 & $-20.47 \pm 0.17$ & $-0.61 \pm 0.15$ & $U B_{\mathrm{AB}} V R_{\mathrm{C}} I_{\mathrm{C}}$ colors \\
\hline & 0.692 & $R_{\mathrm{c}}$ & 21.5 & 0.55 & Late & 1012 & $-20.11 \pm 0.18$ & $-1.34 \pm 0.12$ & to redshifted temp. \\
\hline \multirow[t]{2}{*}{$\mathrm{CNOC} 1$} & - & $r$ & 22.0 & $0.2-0.6$ & 1/2-red & 209 & $-20.60 \pm 0.30$ & $-0.42 \pm 0.28$ & $r-g$ of redshifted \\
\hline & - & $r$ & 22.0 & $0.2-0.6$ & 1/2-blue & 179 & $-20.60 \pm 0.52$ & $-1.47 \pm 0.32$ & non-evolv. Sbc temp. \\
\hline \multirow[t]{3}{*}{ ESS } & 0.247 & $R_{\mathrm{c}}$ & 20.5 & $0.1-0.6$ & Early & 232 & $-20.56 \pm 0.14$ & $0.11 \pm 0.23$ & PCA-spectral class. \\
\hline & 0.247 & $R_{\mathrm{c}}$ & 20.5 & $0.1-0.6$ & Interm & 204 & $-20.43 \pm 0.17$ & $-0.73 \pm 0.19$ & \\
\hline & 0.247 & $R_{\mathrm{c}}$ & 20.5 & $0.1-0.6$ & Late & 181 & $-19.84 \pm 0.24$ & $-1.64 \pm 0.23$ & \\
\hline DARS2 & 70.3 & $I_{\mathrm{c}}$ & $B \leq 17.0$ & 0.06 & ALL & 288 & $-21.92 \pm 0.10$ & $-1.20 \pm 0.12$ & alpha fixed from $B$ LF \\
\hline SDSS & $\sim 2000$ & $i^{*}$ & 16.91 & $0.02-0.22$ & ALL & 88239 & $-21.25 \pm 0.02$ & $-1.03 \pm 0.03$ & \\
\hline
\end{tabular}

Table notes:

- See Table 2 for definition of Cols. All references are provided in the text.

- Wherever necessary, the listed values of $M^{*}$ result from the conversion from the original values derived by the authors in the filters listed in Col. (3), into the Cousins $R_{\mathrm{c}}$ and $I_{\mathrm{c}}$ bands, respectively. The original values of $\alpha$ are kept unchanged.

- $r$ magnitudes are in the Thuan \& Gunn (1976) photometric system.

a The values of $M^{*}$ and $\alpha$ for the CS, SDSS-Morph and COMBO-17 surveys are converted from a cosmology with $\left[\Omega_{\mathrm{m}}=0.3, \Omega_{\lambda}=0.7\right]$ into $\left[\Omega_{\mathrm{m}}=1.0, \Omega_{\lambda}=0.0\right]$ using empirical corrections described in the text. These values should therefore be used with caution.

Comparison of the 4 spectral-type LFs for the COMBO-17 survey with the 3 spectral-type LFs for the ESS and CNOC2 yields similar conclusions as in the $U$ band. For the Type-1 galaxies, the value of $M^{*}$ is consistent with those for the ESS and CNOC2 early-type LFs at less than the 1- $\sigma$ level, whereas the COMBO-17 value of $\alpha$ departs from the values in the ESS and CNOC2 by 1.4- $\sigma$ and 2.6- $\sigma$ respectively. As in the $U$ band, I interpret the systematically larger value of $\alpha$ for the COMBO-17 Type-1 LF as due to: (i) the earlier spectral content of this class, compared to both the ESS and CNOC2 early-type classes; (ii) the likely absence from the COMBO-17 Type- 1 class of dSph galaxies which would flatten the faint-end of the LF (see Sect. 2).

Both Schechter parameters for the COMBO-17 Type-4 LFs are in agreement with those for the ESS and CNOC2 late-type class (at less than the 1- $\sigma$ level, except for $M^{*}$ which differs 
from that for the CNOC2 by $1.5-\sigma)$. This suggests that there is a significant fraction of galaxies in common between the COMBO-17 Type-4 galaxies (with spectral types matching the Starburst templates SB6 to SB1 from Kinney et al. 1996), and the $\mathrm{Sc} / \mathrm{Sm} / \mathrm{Im}$ and $\mathrm{Scd} / \mathrm{Im}$ galaxies selected in the ESS and CNOC2 late-type class respectively. The fractions of galaxies in the corresponding classes for the 3 surveys (29\% in the ESS, $47 \%$ in the $\mathrm{CNOC} 2$, and $52 \%$ in the COMBO-17) also support a significant common population of galaxies.

In contrast, similar differences between the COMBO-17 Type- 2 and Type- 3 LFs and the CNOC2 LFs as those seen in the $U$ band are detected in the $R_{\mathrm{c}}$ band. Both Schechter parameters for the COMBO-17 Type-3 LF (corresponding to spectral types Sbc-SB6) significantly differ from those for the $\mathrm{CNOC} 2$ (Sbc) and the ESS $(\mathrm{Sb} / \mathrm{Sc}$ ) intermediate-type LFs (at the 2.1- $\sigma$ and 2.2- $\sigma$ level resp. for $M^{*}$, at the 3.8- $\sigma$ and 3.1- $\sigma$ level resp. for $\alpha$ ), despite a significant common spectral content (see Sect. 3.1), with offsets in the direction of brighter $M^{*}$ and steeper $\alpha$ for the COMBO-17 LFs. As in the $U$ band, the COMBO-17 Type-2 LF (spectral types Sab-Sbc) is expected to lie in the intermediate region between the early and intermediate-type LF for the CNOC2 and ESS, containing $\mathrm{E} / \mathrm{Sab}$ and $\mathrm{Sbc}$ galaxies respectively. However, for the COMBO-17 Type-2 LF, $M^{*}$ is brighter by $\sim 2.4-\sigma$ and $\alpha$ is steeper by $1.6-\sigma$ and $0.7-\sigma$ than for the CNOC 2 and ESS intermediate-type LFs respectively.

As already stated in Sect. 3.1.1, a shift towards bright magnitudes is expected for the COMBO-17 Starburst galaxies, and the bright values of $M^{*}$ for the Type- 2 and Type- 3 LFs could indicate a severe contamination of these 2 classes by Starburst galaxies. The low expected fraction of Starburst galaxies in these 2 classes however suggest that a similar magnitude bias might affect the Spiral galaxies, which dominate the Type-2 and Type- 3 classes. As in the $U$ band, the similar values of $M^{*}$ for the COMBO-17 Type-4 LF and the ESS and CNOC2 latetype LFs, despite the dominating fraction of Starburst galaxies in the Type-4 class, could result from the combination of a systematic brightening affecting the Type-4 LF compensated for by an intrinsic fainter $M^{*}$ than in the ESS and CNOC2 latetype LFs. The complex selection effects inherent to the use of medium-band photometry for redshift measurement and spectral classification do not allow to discard these 2 interpretations. At last, some systematic biases in the COMBO-17 color transformation from the $r^{*}$ to the $R_{\mathrm{c}}$ band (Wolf 2002) might also operate, although the difference between the response curves in the 2 filters is significantly smaller than between the $m_{280}$ and $U$ bands (see Sect. 3.1.1).

The right panel of Fig. 4 also shows the CNOC1 LFs estimated from the 1/2-red and 1/2-blue sub-samples, separated by the redshifted $r-g$ color of an Sbc galaxy. The values of $M^{*}$ and $\alpha$ for the CNOC1 1/2-red sample are intermediate between those for the early and intermediate-type LFs for the ESS and CNOC2 samples, suggesting an agreement with both surveys. The 2 CNOC1 LFs also display the steepening in $\alpha$ for bluer galaxies, to a value comparable to those for the CNOC2 and ESS late-type LFs. Although the CNOC1 LFs fail to detect a significant dimming in $M^{*}$, because of the correlation between $M^{*}$ and $\alpha$, there is a $\sim 0.7^{\mathrm{mag}}$ dimming of the LF bright-end between the 2 CNOC1 samples (see Fig. 3 in Lin et al. 1997). This is to be compared to the $\sim 1.7^{\mathrm{mag}}$ dimming of the LF bright-end between the CNOC2 and ESS early and late-type classes (see Fig. 3 in Lin et al. 1997, and Fig. 7 in de Lapparent et al. 2003b). This behavior is similar to that for the CS $1 / 3$ red and $1 / 3$ blue samples in the $V$ band and illustrated in Fig. 3, and can be attributed to same cause: the bright end of the CNOC1 1/2-red and 1/2-blue LFs are likely dominated by Elliptical and early-type Spiral galaxies resp., which have similar characteristic magnitudes.

\subsection{2. $R_{\mathrm{C}}$ luminosity functions at redshifts $0.01-0.15$}

The left panel of Fig. 4 gathers the few intrinsic LF estimates from redshift surveys with $0.01 \lesssim z_{\max } \lesssim 0.15$. The $R_{\mathrm{c}}$ LFs for the CS are calculated for the same $1 / 3$ red and $1 / 3$ blue subsamples as the $V$ LFs (see Table 3); the values of $M^{*}$ and $\alpha$ for both samples are empirically converted from cosmological parameters $\left[\Omega_{\mathrm{m}}=0.3, \Omega_{\lambda}=0.7\right]$ into $\left[\Omega_{\mathrm{m}}=1.0, \Omega_{\Lambda}=0.0\right]$ by adding 0.1 and 0.03 resp., based on the variations for the full $R$ sample (see Table 2 of Brown et al. 2001; see also comments on these shifts in Sect. 3.1.1). The resulting LFs display a similar behavior to both the CS $V$ LFs, and the CNOC1 LFs converted into the $R_{\mathrm{c}}$ band. The value of $M^{*}$ dims by only $0.2^{\mathrm{mag}}$ from the CS $1 / 3$ red to the $1 / 3$ blue $R_{\mathrm{c}} \mathrm{LF}$, corresponding to a $0.5^{\mathrm{mag}}$ shift between the bright-end of the 2 LFs. The values of $\alpha$ show the usual steepening from the red to blue sample, and the values are in agreement with those for the CNOC1 (right panel of Fig. 4) at less that the 1- $\sigma$ level.

The only measures of LF in a red filter based on morphological types were recently obtained from a sub-sample with $r^{*} \leq 15.9$ from the Early Data Release (EDR) of the Sloan Digital Sky Survey (Nakamura et al. 2003, denoted here SDSS-Morph). Following Sect. 3.1.1, I convert the listed values of $M^{*}\left(r^{*}\right)$ from $\left[\Omega_{\mathrm{m}}=0.3, \Omega_{\Lambda}=0.7\right]$ to $\left[\Omega_{\mathrm{m}}=1.0, \Omega_{\Lambda}=0.0\right]$ using $\Delta M \simeq 0.06^{\mathrm{mag}}$, which corresponds to the change in absolute magnitude due to the change in luminosity distance at $z \simeq 0.05$, close to the median redshift of the sub-sample. Using the relation $\Delta \alpha \simeq \Delta M / 3$, derived from the various LFs listed in Table 2 of Blanton et al. (2001), I also apply the empirical shift $\Delta \alpha \simeq 0.02$ to the values of $\alpha$ listed by Nakamura et al. (2003). For the 4 SDSS morphological types listed in Table 3, I convert the "cosmology-corrected" values of $M^{*}\left(r^{*}\right)$ into the Cousins $R_{\mathrm{c}}$ band using the 0.24 average $r^{*}-R_{\mathrm{c}}$ color over listed types $\mathrm{E}$ and S0, and the 0.24, 0.23, 0.17 colors for listed types Sab, Sbc, and $\mathrm{Im}$ respectively (Fukugita et al. 1995, in their Table 3 ). The resulting values of $M^{*}\left(R_{\mathrm{c}}\right)$ and $\alpha$ are listed here in Table 3. Note that the LF for types Im is only given by Nakamura et al. (2003) as indicative (hence the lack of error bars), as this sample is too small and too incomplete to provide a reliable LF.

The intrinsic LFs derived by Nakamura et al. (2003) show a nearly flat slope for the 3 morphological types E/S0,S0/Sa/Sb, $\mathrm{Sbc/Sd}$. Only the LF for morphological type Im shows a steep slope $\alpha \sim-1.9$. This is comparable with the behavior of the morphological-type LFs measured in the Johnson $B$ band for the NOG (Marinoni et al. 1999), the CfA2S (Marzke et al. 1994a) and the SSRS2 (Marzke et al. 1998) surveys (described 
in Sect. 3.3.4). The flat faint-end slopes measured by Nakamura et al. (2003) for types E/SO with no evidence of a faint-end decline is at variance with the Gaussian LFs measured locally for E and S0 (see Sect. 2). When Nakamura et al. (2003) use the concentration index for classifying galaxies, they obtain a similar flat early-type LF. The authors interpret this flat slope as the presence of many intrinsically faint elliptical galaxies with a "hard core" out to $M\left(r^{*}\right) \sim-19$. This is in agreement with the fact that in the Virgo cluster, the bright-end of the dSph LF is dominated by nucleated dE (see Figs. 6 and 15 in Sandage et al. 1985). Objects of this type are likely to appear as elliptical galaxies in the visual classification by Nakamura et al. (2003). As stated by the authors, separating the contribution from dSph at the faint-end of the E/SO LF might yield a decline of this LF.

Because the surface brightness profile of dSph galaxies deviates from the $r^{1 / 4}$ profile of giant $\mathrm{E}$ (de Vaucouleurs 1948) and resembles the exponential profile measured for disk galaxies (Binggeli \& Cameron 1991; Binggeli \& Jerjen 1998), I suggest that Nakamura et al. (2003) might have classified some non-nucleated dE galaxies as faint Spiral galaxies. This would explain the absence of a decline in the S0/Sa/Sb LF at $M\left(r^{*}\right)$ fainter than $\sim-19$, as would be expected by combination of the Gaussian LFs measured locally for these 3 galaxies types (see Sect. 2). A contribution from a Schechter LF for dSph with a steep faint-end slope $\alpha \lesssim-1.3$ could explain the increase of the $\mathrm{S} 0 / \mathrm{Sa} / \mathrm{Sb} \mathrm{LF}$ at faint magnitude, with $\alpha=-1.15 \pm 0.26$ (see de Lapparent et al. 2003b). Moreover, the faint boundary $M\left(r^{*}\right) \simeq-18$ of the 3rd SDSS-Morph class (Nakamura et al. 2003) is too bright to show a decrease at faint magnitudes, as this LF is expected to correspond to the combination of the Gaussian LFs for the Sbc and Sd (see Sect. 2). Although the dwarf Irregular galaxies (dI) mostly populate the latest class of the SDSS-Morph sample (Im), an additional contribution at $-20 \lesssim M\left(r^{*}\right) \lessgtr-18$ from dI galaxies (see Table 1 and Fig. 1 in Sect. 2) might contribute to preventing a decline of the faint-end LF for $\mathrm{Sbc/Sd}$ galaxies.

Contrary to the nearby surveys based on morphological types (see Sect. 3.3.4), the SDSS-Morph survey does detect the dimming of $M^{*}$ of the Im galaxies, compared to the earlier classes. However, the value of $M^{*}\left(R_{\mathrm{c}}\right)=-20.11$ for the Schechter Im LF is 2 to $4^{\text {mag }}$ brighter than the values measured from the Centaurus and Virgo cluster (see Table 6 of de Lapparent et al. 2003b), and $\sim 2.5^{\text {mag }}$ brighter than the value derived from the ESO-Sculptor (see Table 7 of de Lapparent et al. 2003b). Nakamura et al. (2003) warn that the SDSSMorph Im sample is incomplete, and it is likely that a significant portion of the "unclassified" objects are faint Im galaxies. Due to the limits of visual classification (Lahav et al. 1995), some type mixing among the $\mathrm{dSph}$, Im and faint Spiral galaxies, might be expected, and could affect the various SDSSMorph LFs.

In the left panel of Fig. 4, I also plot the $R_{\mathrm{c}}$ intrinsic LFs for the Las Campanas Redshift Survey (Bromley et al. 1998; Lin et al. 1996, denoted LCRS). As for the CNOC1, I convert the LCRS Thuan \& Gunn (1976) $r$ magnitudes into the $R_{\mathrm{c}}$ band using the $r-R_{\mathrm{c}}=0.36$ color of an Sbc galaxy (Fukugita et al. 1995, Table 3f), with no distinction of spectral type.
The LCRS intrinsic LFs based on 6 spectral classes derived by a PCA (Bromley et al. 1998), show a smooth variation in $\alpha$ from 0.54 to -1.84 , and a dimming of $M^{*}(R)$ from -20.28 to -20.01 between Clan-1 and Clan-6. The large value $\alpha=$ $0.54 \pm 0.14$ for the Clan-1 LF suggest that this sub-sample contains only early-type galaxies and is not contaminated by dwarf spheroidal galaxies. Further comparison of the LCRS LFs with those for the other surveys is hindered by the fact that Bromley et al. (1998) do not provide the correspondence between their PCA-spectral type and the Hubble morphological types.

Moreover, the LCRS redshift survey is based on multi-fiber spectroscopy for which a spectral classification is subject to biases caused by:

- the relatively small angular size of the fibers $\left(3.5^{\prime \prime}\right)$ compared to the apparent galaxy size, which introduces systematic color biases into the spectra (this effect is called "aperture bias"): color gradients are present in galaxies of varying types (Segalovitz 1975; Boroson \& Thompson 1987; Vigroux et al. 1988; Balcells \& Peletier 1994), and in most cases correspond to several tenths of a magnitude bluer colors when going from the central to the outer regions of a galaxy;

- the astrometric uncertainties which cause an offset of the positioned fiber with respect to the galaxy peak of light;

- the poor flux calibration of the spectra, as the variations in the fiber transmission cannot be accurately calibrated, implying some dispersion in the spectra continuum shape.

To partly overcome the flux-calibration inaccuracy, Bromley et al. (1998) apply to each spectrum a high-pass filter, which effectively removes the continuum of the spectra. The PCA analysis therefore only accounts for "local" features such as the CaII H\&K break, and the absorption and emission lines. Galaz \& de Lapparent (1998) however show that in a spectral classification based on flux-calibrated spectra, the dominant signal originates from the shape of the continuum. Moreover, the lack of accurate flux-calibration in the LCRS also results in significant dispersion in the relative line intensities, likely to cause some contamination among the LCRS spectral classes (no error analysis of the random and systematic errors in the flux-calibration of the LCRS data is however reported by Bromley et al. 1998). The net effect is to smooth the variations among the intrinsic LFs. This could explain the smaller variation in $M^{*}$ between Clan-1 and Clan-6 $\left(0.27^{\mathrm{mag}}\right)$, compared to a variation between the early and late-type LFs of $0.62^{\mathrm{mag}}$ in the ESS, and $0.39^{\mathrm{mag}}$ in the CNOC2. Kochanek et al. (2001) also showed how aperture biases may artificially steepen the LF by mixing galaxies having Schechter LFs with similar faint-end slopes but different $M^{*}$ and different amplitudes. The LFs derived by Bromley et al. (1998) must therefore be used with caution. In Sect. 3.3.2 below, I show that the various biases mentioned here may also affect the $B$ band determinations of the LFs provided by surveys based on multi-fiber spectroscopy.

Left panel of Fig. 4 also shows the intrinsic LFs estimated from the LCRS using the sub-samples with $E W[\mathrm{OII}]>$ $5 \AA$ and $E W[\mathrm{OII}]<5 \AA$ resp. (Lin et al. 1996). Although the 
$\mathrm{H} \alpha \lambda 6563$ emission line is a more reliable indicator of starformation than the [OII] $\lambda 3727$ line as it is less affected by dust and metallicity (Tresse et al. 1999), the [OII] line is often used at $z \gtrsim 0.3$ where the $\mathrm{H} \alpha$ lines shifts into the infrared. The 2 LCRS LFs based on EW[OII] show the similar dimming in $M^{*}$ and steepening in $\alpha$ as seen between the LFs for Clan-2 and an intermediate LF between those for Clan- 4 and Clan-5. Emission lines provide a convenient and straightforward method for separating galaxies with early and late morphological types, as nebular lines result from gas heating by young stars and are thus present in galaxies with current star formation, which in turn tend to be of later morphological type (see Fig. 5, described below). Although the correlations between strength of the nebular lines, the galaxy color/spectraltype and the morphological type suffer some dispersion, they are observed in all galaxy samples (see Figs. 2 and 3 in de Lapparent et al. 2003b). For example, Heyl et al. (1997) show that the evolution detected by Ellis et al. (1996) in the Autofib star-forming galaxies from $z \simeq 0.5$ to the present time (a decrease in luminosity density with decreasing redshift), can be interpreted in terms of evolution in the late-type Spiral galaxies (see Sect. 3.3.3 for analysis of the Autofib intrinsic LFs).

I now show that LF estimates based on $E W[\mathrm{OII}]$ suffer analogous type mixing as those derived from color samples (see Fig. 3). In Fig. 5, I plot the equivalent width of the [OII] emission line as a function of PCA spectral type $\delta$ for the galaxies with $R_{\mathrm{c}} \leq 20.5$ in the ESS. The sample with $E W[\mathrm{OII}]<5 \AA$ contains $72.6 \%, 24.6 \%$, and $2.8 \%$ of early-type, intermediate-type, and late-type galaxies resp., and the sample with $E W[\mathrm{OII}]>5 \AA, 9.9 \%, 38.9 \%$, and $51.2 \%$ respectively. Therefore, the low [OII]-emission sample is dominated by the early-type galaxies, with a small fraction of intermediate-type galaxies and few late-type galaxies; in contrast, the high [OII]-emission sample is approximately equally dominated by the intermediate and late-type galaxies. Measurement of LFs based on the equivalent width of [OII] emission line then fails to discriminate among the intrinsic LFs per morphological type due to type mixing, similarly to the LFs based on color sub-samples. This could explain why the $R_{\mathrm{c}}$ LFs for the CS $1 / 3$ red and $1 / 3$ blue samples nearly follow the LCRS results based on the [OII] emission line (left panel of Fig. 4). There is however one notable difference with the LFs obtained by using a color cut: the fraction of early-type galaxies in the high [OII]-emission ESS sample is relatively smaller than in the ESS-1/3 blue sample.

The LCRS LFs are also useful for emphasizing the need of multiple galaxy classes for estimating intrinsic LFs. The difference in the LCRS LFs between the 6 samples separated by spectral type and the 2 samples based on the strength of the $[\mathrm{OII}]$ emission line illustrates how a wider variety of LFs is measured when a larger number of classes is used. This is due to the multiplicity of shape for the LFs per morphological type (see Sect. 2). In the $R_{\mathrm{c}}$ band, comparison of the LCRS and CS LFs on one hand, and of the CNOC1 and CNOC2 LFs on the other hand, provides evidence that a minimum of 3 spectral classes is necessary for detecting both the Gaussian LF shape for the E and S0 galaxies (sometimes also including

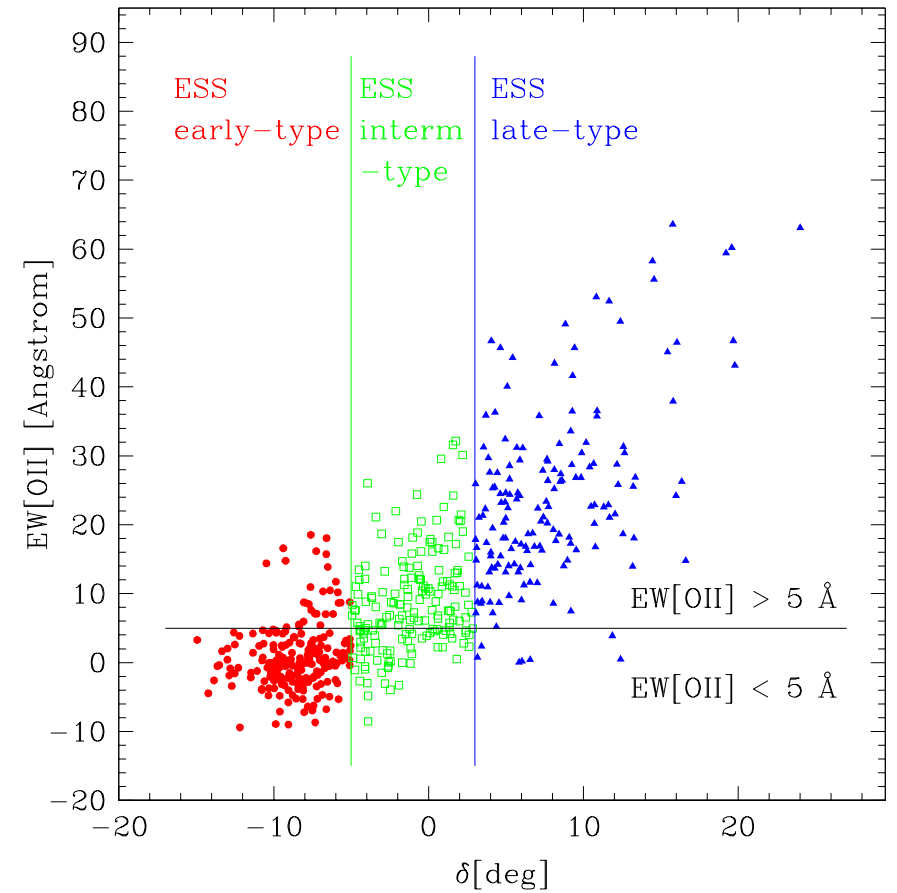

Fig. 5. Relation between the ESO-Sculptor PCA spectral type $\delta$ and the equivalent width of the [OII] emission line for each ESO-Sculptor galaxy. The ESO-Sculptor early, intermediate and late-type galaxies are defined by the intervals $\delta \leq-5.0^{\circ},-5.0<\delta \leq 3.0^{\circ}$, and $\delta>3.0^{\circ}$ resp., separated by the 2 vertical lines. The cut at $E W[\mathrm{OII}]=5 \AA$ used for measurement of the Las Campanas Redshift Survey LFs (Lin et al. $1996)$ is indicated as a horizontal line $(E W[\mathrm{OII}]=5 \AA$ also nearly corresponds to a 2- $\sigma$ significance level in the [OII] line). This graph shows how sub-samples based on $E W[\mathrm{OII}]$ mix galaxies of different spectral types.

$\mathrm{Sa} / \mathrm{Sb}$ galaxies), and the dimming of the late-type Spiral (Sc, $\mathrm{Sd} / \mathrm{Sm}$ ) and the Irregular galaxies compared to earlier-type galaxies (see Fig. 1). Comparison of the ESS and CS LFs in the $V$ band (see Fig. 2 in Sect. 3.1.2) also supports this result.

\section{3. $B$ band}

The most numerous measurements of intrinsic LFs were obtained in the $B$ band. For clarity, Fig. 6 shows the $M^{*}$ and $\alpha$ parameters for samples grouped in four intervals of effective depth $z_{\max }$.

\subsubsection{B luminosity functions at redshifts $0.4-0.6$}

The lower-left panel of Fig. 6 shows the intrinsic LF parameters for the redshift surveys providing measurements at $z_{\max } \sim 0.6$ : the Canada-France Redshift Survey (Lilly et al. 1995, denoted CFRS); the CNOC1 (Lin et al. 1997), CNOC2 (Lin et al. 1999), COMBO-17 (Wolf et al. 2003), and ESS (de Lapparent et al. 2003b), already mentioned in Sects. 3.1.1, 3.1.2 and 3.2; and the Calar Alto Deep Imaging Survey (Fried et al. 2001, denoted CADIS). The photometric catalogues on which are based all these redshift surveys are obtained from CDD imaging (see Table 4 for the sample parameters). 


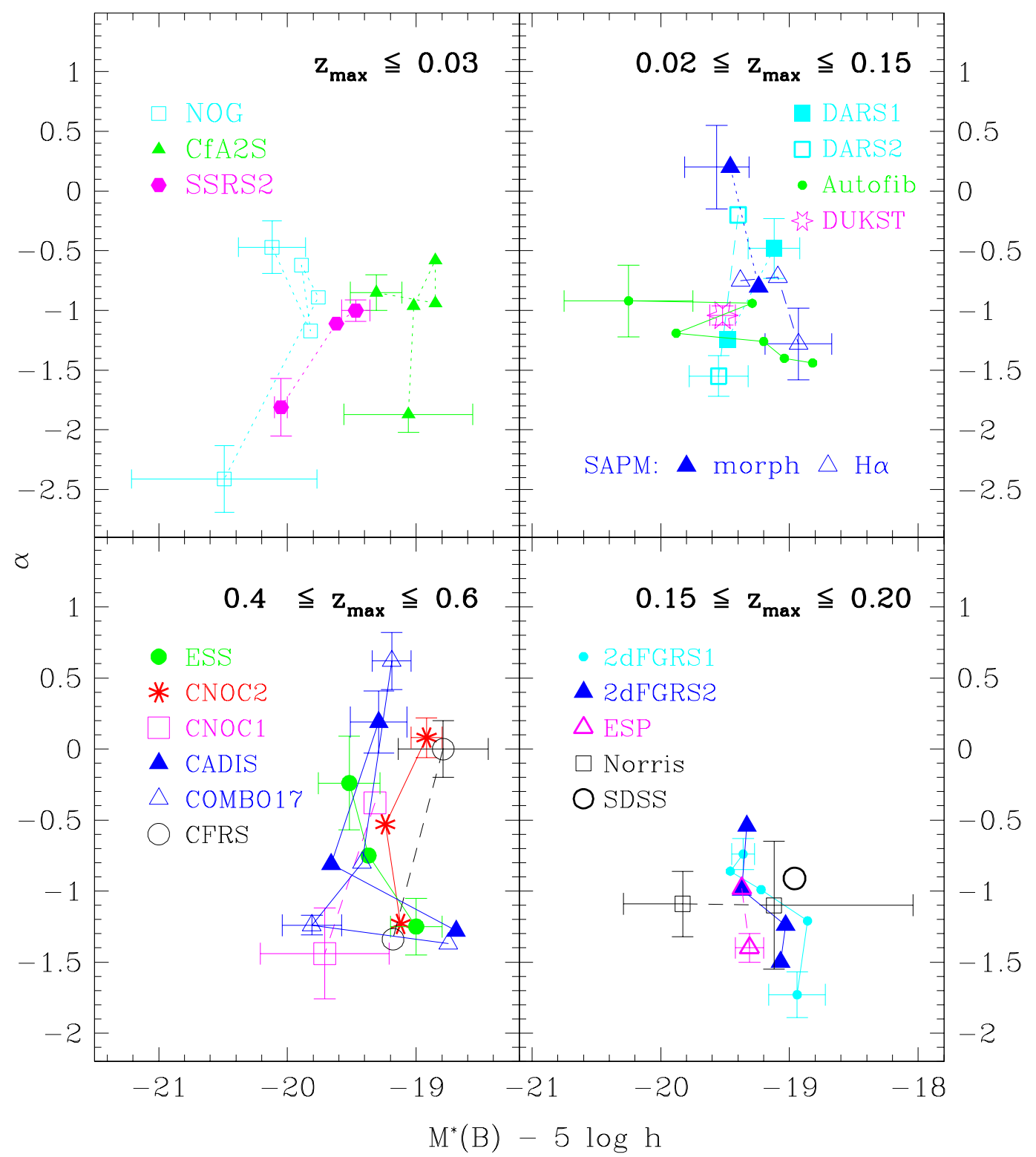

Fig. 6. Comparison of the Schechter parameters $M^{*}$ and $\alpha$ for the existing intrinsic LFs measured or converted into the Johnson $B$ band. Other existing surveys providing only a general LF are also indicated (DUKST in the upper-right panel; SDSS in the lower-right panel). As the error bars for the 2dFGRS2 and SDSS surveys (lower-right panel) are smaller than the symbol size (Table 4), they are not plotted. The 4 panels are arranged by increasing effective depth $z_{\max }$, starting from the upper-left panel and moving clock-wise; the interval of effective depth is indicated in each panel. Solid, dotted, and dashed lines connect the various classes of a given survey when these are based on spectral types, morphological types, and a color cut or the equivalent width of emission lines, respectively. For all surveys, galaxies of later spectral/morphological type or with stronger emission lines are in the direction of steeper slopes $\alpha$ (towards negative values), except for the Norris survey in the lower-right panel, for which $\alpha$ is nearly constant: the "late-type" galaxies are those with the fainter $M^{*}$. For clarity, the error bars in the lower-left panel are only shown for either the early-type point or the late-type point of each survey, or for both points; error bars for the other points are comparable, except in the COMBO-17, with similar error bars for the Type-2, Type-3 and Type-4 LFs, and in the ESS, with similar error bars for the intermediate-type and late-type LFs (see Table 4).

As in the $U$ and $R_{\mathrm{c}}$ bands, I use the listed values of $M^{*}(z=0.3)$ for the CNOC2 $B$ LFs (Lin et al. 1999), and the intrinsic LFs in the interval $0.2 \lessgtr z \lesssim 0.4$ for the COMBO-17 survey (Wolf et al. 2003). As in the $V$ and $R_{\mathrm{c}}$ bands, I use for the ESS the values of $M^{*}$ and $\alpha$ derived from the full redshift range of the survey $(0.1 \leq z \leq 0.6)$. Both the CADIS and CFRS measure LFs in the intervals $0.5 \lesssim z \lesssim 0.75$ and $0.75 \lesssim z \lesssim 1.0$, in which they detect evolutionary effects using the LFs with $z \leq 0.5$ as reference. I however use their intrinsic LFs derived in the intervals $0.3 \leq z \leq 0.5$ and $0.2 \leq z \leq 0.5$ resp., because these provide the best constraint on $\alpha$ for each survey (see Fried et al. 2001; Lilly et al. 1995); these redshift intervals also correspond to $z_{\max } \lesssim 0.6$. For the CFRS, the values of $M^{*}$, listed for $h=0.5$ (Lilly et al. 1995), are converted to $h=1$. 
Table 4. Schechter parameters for the $B$ intrinsic or general LFs measured from the existing redshift surveys.

\begin{tabular}{|c|c|c|c|c|c|c|c|c|c|}
\hline $\begin{array}{l}\text { Survey } \\
\text { (1) }\end{array}$ & $\begin{array}{r}\text { Area } \\
(2)\end{array}$ & $\begin{array}{l}\lambda \\
(3)\end{array}$ & $\begin{array}{l}m_{\lim } \\
(4)\end{array}$ & $\begin{array}{r}z \\
(5)\end{array}$ & $\begin{array}{l}\text { Class } \\
(6)\end{array}$ & $\begin{array}{r}N_{\text {gal }} \\
\text { (7) }\end{array}$ & $\begin{array}{c}M^{*}-5 \log h \\
(8)\end{array}$ & $\begin{array}{c}\alpha \\
(9)\end{array}$ & $\begin{array}{l}\text { Comment } \\
\text { (10) }\end{array}$ \\
\hline \multirow[t]{5}{*}{ NOG } & 27140 & $B$ & 14.0 & 0.02 & $\mathrm{E}$ & 344 & $-20.12 \pm 0.26$ & $-0.47 \pm 0.22$ & morphological class. \\
\hline & 27140 & $B$ & 14.0 & 0.02 & So & 596 & $-19.82 \pm 0.26$ & $-1.17 \pm 0.20$ & \\
\hline & 27140 & $B$ & 14.0 & 0.02 & $\mathrm{Sa}-\mathrm{Sb}$ & 1521 & $-19.89 \pm 0.12$ & $-0.62 \pm 0.11$ & \\
\hline & 27140 & $B$ & 14.0 & 0.02 & Sc-Sd & 2240 & $-19.76 \pm 0.11$ & $-0.89 \pm 0.10$ & \\
\hline & 27140 & $B$ & 14.0 & 0.02 & Sm-Im & 619 & $-20.49 \pm 0.72$ & $-2.41 \pm 0.28$ & \\
\hline \multirow[t]{5}{*}{ CfA2S } & 1370 & $B_{\mathrm{Zw}}$ & 15.5 & 0.03 & $\mathrm{E}$ & - & $-19.31 \pm 0.20$ & $-0.85 \pm 0.15$ & morphological class. \\
\hline & 1370 & $B_{\mathrm{Zw}}$ & 15.5 & 0.03 & So & - & $-18.85 \pm 0.10$ & $-0.94 \pm 0.10$ & \\
\hline & 1370 & $B_{\mathrm{Zw}}$ & 15.5 & 0.03 & $\mathrm{Sa}-\mathrm{Sb}$ & - & $-18.85 \pm 0.10$ & $-0.58 \pm 0.10$ & \\
\hline & 1370 & $B_{\mathrm{Zw}}$ & 15.5 & 0.03 & Sc-Sd & - & $-19.02 \pm 0.15$ & $-0.96 \pm 0.10$ & \\
\hline & 1370 & $B_{\mathrm{Zw}}$ & 15.5 & 0.03 & Sm-Im & - & $-19.06 \pm 0.50$ & $-1.87 \pm 0.15$ & \\
\hline \multirow[t]{3}{*}{ SSRS2 } & 5550 & $B_{\mathrm{Zw}}$ & 15.5 & 0.03 & E-SO & 1587 & $-19.47 \pm 0.11$ & $-1.00 \pm 0.09$ & morphological class. \\
\hline & 5550 & $B_{\mathrm{Zw}}$ & 15.5 & 0.03 & Spiral & 3227 & $-19.62 \pm 0.08$ & $-1.11 \pm 0.07$ & \\
\hline & 5550 & $B_{\mathrm{Zw}}$ & 15.5 & 0.03 & Irr-Pec & 204 & $-20.05 \pm 0.05$ & $-1.81 \pm 0.24$ & \\
\hline \multirow[t]{2}{*}{ DARS1 } & 70.3 & $b_{\mathrm{J}}$ & 17.0 & 0.06 & E-S0 & 97 & $-19.12 \pm 0.20$ & $-0.48 \pm 0.25$ & morphological class. \\
\hline & 70.3 & $b_{\mathrm{J}}$ & 17.0 & 0.06 & Sp-Irr & 194 & $-19.48 \pm 0.20$ & $-1.24 \pm 0.25$ & \\
\hline \multirow[t]{2}{*}{ DARS2 } & 70.3 & $B$ & 17.0 & 0.06 & 1/2-red & 144 & $-19.40 \pm 0.17$ & $-0.20 \pm 0.26$ & $(U-B)_{\text {rest }}>0.2$ \\
\hline & 70.3 & $B$ & 17.0 & 0.06 & $1 / 2$-blue & 144 & $-19.55 \pm 0.23$ & $-1.55 \pm 0.17$ & $(U-B)_{\text {rest }}<0.2$ \\
\hline DUKST & 1460 & $b_{\mathrm{J}}$ & 17.0 & 0.07 & ALL & 2500 & $-19.52 \pm 0.10$ & $-1.04 \pm 0.08$ & \\
\hline \multirow[t]{5}{*}{ SAPM $^{\mathrm{a}}$} & 4300 & $b_{\mathrm{J}}$ & 17.15 & 0.07 & E-S0 & 311 & $-19.46 \pm 0.25$ & $0.20 \pm 0.35$ & morphological class. \\
\hline & 4300 & $b_{\mathrm{J}}$ & 17.15 & 0.07 & Sp-Irr & 999 & $-19.24 \pm 0.16$ & $-0.80 \pm 0.20$ & \\
\hline & 4300 & $b_{\mathrm{J}}$ & 17.15 & 0.07 & $\mathrm{H} \alpha$-low & 599 & $-19.38 \pm 0.24$ & $-0.75 \pm 0.28$ & $E W(\mathrm{H} \alpha)<2 \AA$ \\
\hline & 4300 & $b_{\mathrm{J}}$ & 17.15 & 0.07 & $\mathrm{H} \alpha$-mid & 473 & $-19.09 \pm 0.23$ & $-0.72 \pm 0.29$ & $2<E W(\mathrm{H} \alpha)<15 \AA$ \\
\hline & 4300 & $b_{\mathrm{J}}$ & 17.15 & 0.07 & $\mathrm{H} \alpha$-high & 459 & $-18.93 \pm 0.26$ & $-1.28 \pm 0.30$ & $E W(\mathrm{H} \alpha)>15 \AA$ \\
\hline \multirow[t]{6}{*}{ Autofib } & 10.2 & $b_{\mathrm{J}}$ & 24.0 & $0.02-0.15$ & Red-E & 154 & -20.25 & -0.92 & spectral class. by \\
\hline & 10.2 & $b_{\mathrm{J}}$ & 24.0 & $0.02-0.15$ & Blue-E & 177 & -19.29 & -0.94 & cross-correlation \\
\hline & 10.2 & $b_{\mathrm{J}}$ & 24.0 & $0.02-0.15$ & $\mathrm{Sab}$ & 282 & -19.88 & -1.19 & \\
\hline & 10.2 & $b_{\mathrm{J}}$ & 24.0 & $0.02-0.15$ & Sbc & 361 & -19.20 & -1.26 & \\
\hline & 10.2 & $b_{\mathrm{J}}$ & 24.0 & $0.02-0.15$ & Scd & 539 & -19.04 & -1.40 & \\
\hline & 10.2 & $b_{\mathrm{J}}$ & 24.0 & $0.02-0.15$ & Sdm & 90 & -18.82 & -1.44 & includes Starburst \\
\hline \multirow[t]{5}{*}{ 2dFGRS1 } & $\sim 60$ & $b_{\mathrm{J}}$ & 19.45 & 0.15 & Type-1 & 1850 & $-19.36 \pm 0.09$ & $-0.74 \pm 0.11$ & PCA-spectral class. \\
\hline & $\sim 60$ & $b_{\mathrm{J}}$ & 19.45 & 0.15 & Type- 2 & 958 & $-19.46 \pm 0.14$ & $-0.86 \pm 0.15$ & \\
\hline & $\sim 60$ & $b_{\mathrm{J}}$ & 19.45 & 0.15 & Type-3 & 1200 & $-19.22 \pm 0.12$ & $-0.99 \pm 0.13$ & \\
\hline & $\sim 60$ & $b_{\mathrm{J}}$ & 19.45 & 0.15 & Type-4 & 1193 & $-18.86 \pm 0.12$ & $-1.21 \pm 0.12$ & \\
\hline & $\sim 60$ & $b_{\mathrm{J}}$ & 19.45 & 0.15 & Type-5 & 668 & $-18.94 \pm 0.22$ & $-1.73 \pm 0.16$ & \\
\hline \multirow[t]{4}{*}{ 2dFGRS2 } & $\sim 1200$ & $b_{\mathrm{J}}$ & 19.45 & 0.15 & Type-1 & 27540 & $-19.33 \pm 0.05$ & $-0.54 \pm 0.02$ & PCA-spectral class. \\
\hline & $\sim 1200$ & $b_{\mathrm{J}}$ & 19.45 & 0.15 & Type-2 & 24256 & $-19.37 \pm 0.03$ & $-0.99 \pm 0.01$ & \\
\hline & $\sim 1200$ & $b_{\mathrm{J}}$ & 19.45 & 0.15 & Type-3 & 15016 & $-19.03 \pm 0.04$ & $-1.24 \pm 0.02$ & \\
\hline & $\sim 1200$ & $b_{\mathrm{J}}$ & 19.45 & 0.15 & Type-4 & 8386 & $-19.07 \pm 0.05$ & $-1.50 \pm 0.03$ & \\
\hline \multirow[t]{2}{*}{ ESP } & 23.2 & $b_{\mathrm{J}}$ & 19.4 & 0.15 & no-emi & 1767 & $-19.37 \pm 0.10$ & $-0.98 \pm 0.09$ & $E W<5 \AA$ \\
\hline & 23.2 & $b_{\mathrm{J}}$ & 19.4 & 0.15 & emi-line & 1575 & $-19.31 \pm 0.11$ & $-1.40 \pm 0.10$ & $E W>5 \AA$ \\
\hline SDSS & $\sim 2000$ & $g^{*}$ & 17.69 & $0.02-0.17$ & ALL & 53999 & $-18.96 \pm 0.02$ & $-0.90 \pm 0.03$ & \\
\hline \multirow[t]{2}{*}{ Norris } & 25.0 & $B_{\mathrm{AB}}$ & $r \leq 20.0$ & $0.0-0.2$ & no-OII & 159 & $-19.83 \pm 0.46$ & $-1.09 \pm 0.23$ & $E W[\mathrm{OII}]<10 \AA$ \\
\hline & 25.0 & $B_{\mathrm{AB}}$ & $r \leq 20.0$ & $0.0-0.2$ & OII & 60 & $-19.12 \pm 1.08$ & $-1.10 \pm 0.45$ & $E W[\mathrm{OII}]>10 \AA$ \\
\hline \multirow[t]{4}{*}{$\mathrm{COMBO}^{\mathrm{b}}$} & 0.78 & $B$ & $R \lessgtr 24.0$ & $0.2-0.4$ & Type-1 & 344 & $-19.19 \pm 0.15$ & $0.62 \pm 0.20$ & fits of obs. SEDs \\
\hline & 0.78 & $B$ & $R \lessgtr 24.0$ & $0.2-0.4$ & Type-2 & 986 & $-19.42 \pm 0.17$ & $-0.80 \pm 0.08$ & of redshifted temp. \\
\hline & 0.78 & $B$ & $R \lessgtr 24.0$ & $0.2-0.4$ & Type-3 & 1398 & $-19.81 \pm 0.23$ & $-1.24 \pm 0.07$ & \\
\hline & 0.78 & $B$ & $R \lesssim 24.0$ & $0.2-0.4$ & Type-4 & 2946 & $-18.75 \pm 0.16$ & $-1.37 \pm 0.06$ & \\
\hline \multirow[t]{2}{*}{ CFRS } & 0.0347 & $B_{\mathrm{AB}}$ & $I_{\mathrm{AB}}<22.5$ & $0.2-0.5$ & 1/2-red & - & $-18.79 \pm 0.35$ & $0.00 \pm 0.20$ & $(V-I)_{\mathrm{AB}}$ of redshifted \\
\hline & 0.0347 & $B_{\mathrm{AB}}$ & $I_{\mathrm{AB}}<22.5$ & $0.2-0.5$ & 1/2-blue & - & $-19.18 \pm 0.35$ & $-1.34 \pm 0.20$ & non-evolv. Sbc temp. \\
\hline \multirow[t]{3}{*}{ CADIS } & 0.0833 & $B$ & $I_{815} \lesssim 23$ & $0.3-0.5$ & $\mathrm{E}-\mathrm{Sa}$ & 82 & $-19.29 \pm 0.22$ & $0.19 \pm 0.22$ & fit of obs. SEDs \\
\hline & 0.0833 & $B$ & $I_{815} \lesssim 23$ & $0.3-0.5$ & $\mathrm{Sa}-\mathrm{Sc}$ & 301 & $-19.66 \pm 0.30$ & $-0.81 \pm 0.13$ & to redshifted temp. \\
\hline & 0.0833 & $B$ & $I_{815} \lesssim 23$ & $0.3-0.5$ & Starburst & 252 & $-18.69 \pm 0.29$ & $-1.28 \pm 0.21$ & \\
\hline $\mathrm{CNOC} 2$ & 0.692 & $B_{\mathrm{AB}}$ & $R_{\mathrm{c}}<21.5$ & 0.55 & Early & 611 & $-18.92 \pm 0.12$ & $0.08 \pm 0.14$ & least-square fit of obs. \\
\hline & 0.692 & $B_{\mathrm{AB}}$ & $R_{\mathrm{c}}<21.5$ & 0.55 & Interm & 518 & $-19.24 \pm 0.16$ & $-0.53 \pm 0.15$ & $U B_{\mathrm{AB}} V R_{\mathrm{C}} I_{\mathrm{C}}$ colors \\
\hline & 0.692 & $B_{\mathrm{AB}}$ & $R_{\mathrm{c}}<21.5$ & 0.55 & Late & 1016 & $-19.12 \pm 0.16$ & $-1.23 \pm 0.12$ & to redshifted temp. \\
\hline CNOC1 & - & $B_{\mathrm{AB}}$ & $r \leq 22.0$ & $0.2-0.6$ & 1/2-red & 209 & $-19.32 \pm 0.30$ & $-0.38 \pm 0.29$ & $r-g$ of redshifted \\
\hline & - & $B_{\mathrm{AB}}$ & $r \leq 22.0$ & $0.2-0.6$ & 1/2-blue & 180 & $-19.71 \pm 0.50$ & $-1.44 \pm 0.32$ & non-evolv. Sbc temp. \\
\hline ESS & 0.219 & $B$ & 22.0 & $0.1-0.6$ & Early & 108 & $-19.52 \pm 0.24$ & $-0.24 \pm 0.33$ & PCA-spectral class. \\
\hline & 0.219 & $B$ & 22.0 & $0.1-0.6$ & Interm & 154 & $-19.37 \pm 0.20$ & $-0.75 \pm 0.21$ & \\
\hline & 0.219 & $B$ & 22.0 & $0.1-0.6$ & Late & 190 & $-19.00 \pm 0.20$ & $-1.25 \pm 0.20$ & \\
\hline
\end{tabular}

Table notes:

- See Table 2 for definition of columns. All references are provided in the text.

- All listed values of $M^{*}$ result from the conversion from the original values derived by the authors in the filters listed in column (3), into the Johnson $B$ band. The original values of $\alpha$ are kept unchanged.

- All quoted $b_{\mathrm{J}}$ magnitudes are from photographic plates.

a In the survey denoted SAPM, sub-sample H $\alpha$-low contains 233 E-S0, 217 Sp-Irr, 149 unclassified galaxies; sub-sample H $\alpha$-mid, 24 E-S0, $358 \mathrm{Sp}$-Irr, and 81 unclassified galaxies; sub-sample $\mathrm{H} \alpha$-high, $20 \mathrm{E}-\mathrm{S} 0,344 \mathrm{Sp}$-Irr, and 95 unclassified galaxies.

b The values of $M^{*}$ and $\alpha$ for the COMBO-17 survey are converted from a cosmology with $\left[\Omega_{\mathrm{m}}=0.3, \Omega_{\lambda}=0.7\right]$ into $\left[\Omega_{\mathrm{m}}=1.0, \Omega_{\lambda}=0.0\right]$ using empirical corrections described in the text. These values should therefore be used with caution. 
Note that no uncertainties are quoted by Lilly et al. (1995) for the Schechter parameters of the CFRS 1/2-red and 1/2-blue LFs. In Table 6, I have approximated these uncertainties as $\sqrt{2}$ times the uncertainties $\sigma(M)=0.25$ and $\sigma(\alpha)=$ 0.15 quoted for the general LF in the interval $0.2 \leq z \leq 0.5$ (see Sect. 3.1.1 in Lilly et al. 1995). The values of $M^{*}$ for the CNOC2, CNOC1, and CFRS are measured in $B_{\mathrm{AB}}$ : I convert them into Johnson $B$ magnitudes using $B-B_{\mathrm{AB}}=0.14$, as estimated by Fukugita et al. (1995).

I first compare the LFs for the 3 surveys which are based on a spectral classification, and are split into 3 spectral classes: the ESS, CNOC2 and CADIS. Despite selection effects specific to each sample, Fig. 6 shows that the $B$ band intrinsic LFs for the 3 surveys have a similar behavior. All 3 surveys show the steepening in $\alpha$ from early to late-type classes: the slope $\alpha$ increases from values inside the interval $-0.24 \leq \alpha \leq 0.19$ for the earlytype galaxies, to the narrow range $-1.28 \leq \alpha \leq-1.23$ for the late-type galaxies. The values of $M^{*}$ also describe narrow intervals of $\lesssim 0.6^{\mathrm{mag}}$ among the 3 surveys for each of the 3 classes. If one assumes that the 3 spectral classes in the ESS, CNOC2 and CADIS sample similar galaxy populations, taken 2 by 2 , the ESS and CADIS LFs, and the CNOC2 and CADIS LFs are in agreement at the 1- $\sigma$ level.

There are however 2 noticeable differences between the CNOC2 and ESS $B$ LFs. First, there is a 2- $\sigma$ difference between the $M^{*}$ values for the CNOC2 and ESS early-type LFs. I also note that there is only a very small dimming of the bright-end of the CNOC2 LF from early to late types: a shift of approximately $0.5^{\mathrm{mag}}$ towards faint magnitudes is however evaluated from Fig. 5 of Lin et al. (1999); it converts into a $0.2^{\mathrm{mag}}$ brightening of $M^{*}(B)$ from early to late types because of the correlation between $M^{*}$ and $\alpha$ (see Sect. 3.1.2). In contrast, there is $\mathrm{a} \simeq 1.0^{\mathrm{mag}}$ shift towards faint magnitudes of the bright-end of both the ESS and CADIS $B$ LFs from early to late types, which is measured by a dimming in $M^{*}(B)$ of $0.52^{\mathrm{mag}}$ in the ESS and $0.60^{\mathrm{mag}}$ in the CADIS. These effects could be due to the incompleteness of the ESS $B$ sample, and a difference in morphological type content of the spectral classes in the 3 surveys.

Because the spectral classification and redshift measurement in the CADIS and COMBO-17 surveys are based on a similar technique (medium-band photometry; see Sect. 3.1.1 and below), it is useful to compare the results from the 2 surveys. The major differences between the 2 surveys are the larger statistic for the COMBO-17 and its use of 4 spectral classes, compared to 3 spectral classes in the CADIS (see Table 4; there is also some difference in the set of filters, see below). Lower-left panel of Fig. 6 shows that there is remarkable agreement between the CADIS E-Sa, Sa-Sc, Starburst LFs and the COMBO-17 Type-1 (E-Sa), Type-2 (Sa-Sbc), Type-4 (SB6-SB1) LFs resp., as expected from the similar spectral type content in the corresponding classes. As observed in the ESS and CADIS $B$ LFs, the LF for COMBO-17 Type-4 galaxies shows the dimming of $M^{*}$ due to the expected dominant contribution from dI galaxies in this class. The noticeable brightening in $M^{*}$ for the COMBO-17 $B$ LF for Type-3 (Sbc-SB6) galaxies, compared to the Type- 1 and Type- 2 classes, may be due to the combination of significant mass and star formation rate in $B$ for the galaxies in this class. Here, contrary to the $U$ and $R_{\mathrm{c}}$ bands, there is no need to invoke some systematic bias in the absolute magnitudes (related to the larger redshifts errors for the faint Starburst galaxies). Note that the Johnson $B$ band is the only band considered here in which the COMBO-17 LFs are directly provided. This strengthens the suggestion that the difference in the $U$ and $R_{\mathrm{c}}$ bands between the COMBO-17 Type-2 and Type-3 LFs and the intermediate-class LFs of the CNOC2 and ESS may be due to some biases in the color coefficients required to convert the COMBO-17 $m_{280}$ and $r^{*}$ LFs into the $U$ and $R_{\mathrm{c}}$ bands respectively.

The type content and respective fractions of galaxies in the CADIS classes (13\% E-S0, 47\% Sa-Sc, $40 \%$ Starburst galaxies) and the COMBO-17 classes (6\% Type-1 [E-Sa], 17\% Type-2 [Sa-Sbc], 25\% Type-3 [Sbc-SB6], 52\% Type-4 [SB6-SB1] galaxies) also indicate that the CADIS class $\mathrm{Sa}-\mathrm{Sc}$ is expected to contain a significant part of both the COMBO-17 Type-2 and Type-3 galaxies. Taking the average of the Schechter parameters for the COMBO-17 Type-2 and Type- 3 classes yields values of $M^{*}$ and $\alpha$ in acceptable agreement with the CADIS Sa-Sc class (at less than 1- $\sigma$ level for $M^{*}$, and less than 2- $\sigma$ level for $\alpha$ ). The $B$ LFs for the COMBO-17 therefore demonstrate the gain in information when changing from 3 to 4 spectral classes. This gain is effective because the chosen COMBO-17 classes succeed in separating galaxies with different intrinsic LFs.

The agreement of the CADIS and COMBO-17 $B$ LFs with those for the ESS and CNOC2 demonstrates the interest of the "photometric redshift" approach for measuring LFs: the CADIS survey is based on a combination of 4 wide-band filters $\left(B R J K^{\prime}\right)$ and up to 13 medium-band $(\Delta \lambda \simeq 250-500 \AA)$ filters; the redshifts and spectral types of $\sim 2780$ galaxies were measured using a standard minimization procedure in which the observed SEDs are compared to a spectral library. The resulting redshifts uncertainties are $\sigma(z) \leq 0.03$, to be compared to $\sim 0.0001-0.003$ for the spectroscopic surveys listed in Tables 2-3 (for comparison, the COMBO-17 uses 5 wideband filters -Johnson UBVRI- and 12 medium-band filters with $F W H M \simeq 140-310 \AA$ which yield the same redshift uncertainty as in the CADIS). The $\sigma(z) \leq 0.03$ redshift uncertainty in the CADIS survey is nevertheless sufficient for derivation of spectral-type LFs in agreement with those derived from redshift surveys such as the CNOC2 and ESS. The reason is that the dispersion in the absolute magnitudes caused by the redshift uncertainties, of order of $5 \sigma(z) / z$, are significantly smaller than the width of the LF for each morphological types (see Fig. 1 in Sect. 2): for example, $5 \sigma(z) / z=0.3$ at $z=0.5$ in the CADIS survey, which is nearly 10 times smaller than the dispersion of the Gaussian LFs for the giant galaxies (see Table 1 in Sect. 2); this is even smaller than the 8 mag interval over which the Schechter LFs for dwarf galaxies are defined (see Jerjen et al. 2000; Trentham \& Tully 2002).

The 2-class LFs derived from the CNOC1 and CFRS, also plotted in the lower-left panel of Fig. 6, are based on a color cut at the redshifted color of a non-evolving Sbc galaxy. Both samples confirm the steepening in $\alpha$ for bluer galaxies observed for the ESS, CNOC2, CADIS and COMBO-17 surveys. It is however noticeable that for both the CFRS and the CNOC1, 
$M^{*}$ for the blue sample is $\sim 0.4^{\mathrm{mag}}$ brighter than for the red sample. Examination of the corresponding curves in Lin et al. (1997) and Lilly et al. (1995) shows that this effect is due to the correlation between $M^{*}$ and $\alpha$ in the Schechter parameterization: for the CNOC1, the bright-end of the blue LF is actually fainter by $\sim 0.2-0.3^{\mathrm{mag}}$ than that for the red LF; for the CFRS, the bright-end of the blue LF is not determined, but the few common points with the red LF (those lying around the "knee" at $\left.M\left(B_{\mathrm{AB}}\right) \simeq-19.5\right)$ suggest also a fainter bright-end by $\lesssim 0.5^{\mathrm{mag}}$ for the blue LF. This confirms the $\alpha$-dependent relation mentioned in Sect. 3.1.2 between the bright exponential fall-off of a given LF and the value of $M^{*}$.

The 0.2 to $0.5^{\mathrm{mag}}$ dimming of the LF bright-end from the CNOC1 and CFRS 1/2-red to 1/2-blue samples is however smaller than for the ESS $\left(\sim 1.0^{\mathrm{mag}}\right)$. The small dimming in $M^{*}$ for the CNOC1 and CFRS in the $B$ band is similar to that already described for the CNOC1 LFs in the $r$ band in Sect. 3.2 and attributed to type-mixing: the use of only 2 spectral classes fails in separating the blue low luminosity galaxies from the more luminous Spiral galaxies; the bright ends of the red and blue LFs are dominated by Elliptical and Spiral galaxies resp., which have similar characteristic magnitudes (see Table 1 and Fig. 3); due to the correlation between $M^{*}$ and $\alpha$, combination with a steeper $\alpha$ for the blue LF then results in a brighter $M^{*}$ for that LF. As in the $R_{\mathrm{c}}$ and $V$ bands, comparison of the ESS, CNOC2 and CADIS LFs with those for the CNOC1 and CFRS illustrates the significant gain of information when changing from 2 to 3 spectral classes, due to the multiplicity of shape for the intrinsic LFs of the dominant morphological types (see Sect. 2).

\subsubsection{B luminosity functions at redshifts $0.15-0.20$}

The lower-right panel of Fig. 6 shows the $M^{*}$ and $\alpha$ parameters for the intrinsic LFs measured from redshift surveys with $0.15 \lesssim z_{\max } \lesssim 0.20$ : the first and second sub-samples of the 2dF Galaxy Redshift Survey (2dFGRS) from which intrinsic LFs were derived, based on 5869 galaxies (Folkes et al. 1999, denoted 2dFGRS1), and 75589 galaxies (Madgwick et al. 2002, denoted 2dFGRS2); the ESO Slice Project (Zucca et al. 1997, denoted ESP); the Norris Survey of the Corona Borealis Supercluster (Small et al. 1997). The photometric surveys on which are based all these mentioned redshift surveys are obtained from digitized photographic plates.

For converting the values of $M^{*}$ measured as $b_{\mathrm{J}}$ magnitudes into Johnson $B$ magnitudes for the 2dFGRS1, 2dFGRS2, and ESP, I apply the $B-b_{\mathrm{J}}=0.28(B-V)$ color equation determined by Blair \& Gilmore (1982, see also Norberg et al. 2002) for the UK Schmidt Telescope photographic system, complemented by the $B-V$ colors estimated by Fukugita et al. (1995, Table 3a): for the 5 2dFGRS1 types listed in Table 4, I use the average $B-V$ color 0.905 over listed types $\mathrm{E}$ and S0, and the $B-V$ colors $0.78,0.57,0.50,0.27$, for listed types $S a b$, Sbc, Scd, Im resp.; for the $42 \mathrm{dFGRS} 2$ types, I use the average $B-V$ color 0.905 over listed types E, S0 and Sa, and the $B-V$ colors $0.57,0.50$, and 0.27 , for listed types Sbc, Scd, Im resp.; for the ESP, I use the average $B-V$ color 0.905 over listed types E and S0, and 0.57 for listed type Sbc. The resulting $B-b_{\mathrm{J}}$ colors are 0.25 for the average between listed types E and S0, and 0.22, 0.16, 0.14, 0.08 for listed types Sab, Sbc, Scd, Im resp., which are assigned to the 2dFGRS1; 0.25 for the average between listed types E and S0, and 0.16, 0.14, 0.08 for listed types Sbc, Scd, Im resp., which are assigned to the 2dFGRS2; 0.25 for the average between listed types E and S0, and 0.16 for listed type Sbc, which are assigned to the ESP.

For the Norris survey, I convert values of $M^{*}$ measured in $B_{\mathrm{AB}}$ into Johnson $B$ magnitudes using again $B-B_{\mathrm{AB}}=0.14$, as estimated by Fukugita et al. (1995). Note that the areas of sky sampled by the 2dFGRS1 and 2dFGRS2 are not provided by the authors; I roughly estimate them using the other elements of information provided by the authors (number of fields and number of spectra per field). The resulting approximate areas listed in Table 4 could be in error by as much as a factor 2 .

In the ESP (Zucca et al. 1997), the detection/no-detection of the [OII] $\lambda 3727$ emission line is used for separating the sample into 2 spectral classes; as stated by the authors and indicated in Table 4, detection of emission lines corresponds to a threshold of $5 \AA$ in equivalent width. The resulting LF for the galaxies with no or weak [OII] line has a nearly flat slope in the $B$ band ( $\alpha \simeq-1.0$; the corresponding point in Fig. 6 is overlayed with that for the 2dFGRS2 Type-2 LF), and a steeper slope is measured for the galaxies with strong [OII] $(\alpha \simeq-1.3)$; the variation in $M^{*}$ is small from one sub-sample to the other and within the error bars. As for the LCRS $r$ LFs (Lin et al. 1996) based on the equivalent width of the [OII] line (see Sect. 3.2), I interpret the flat slope of the ESP LF for galaxies with low [OII]emission as the result of type mixing, as demonstrated in Fig. 5 (see Sect. 3.2.2): the LF for that sample is likely to be contaminated at the faint end by Spiral and Irregular galaxies, thus failing to isolate the bounded LFs for E and S0 galaxies. Note however that both ESP LFs in the $B$ band have a steeper value of $\alpha$ by $\sim 0.5$ compared to the corresponding LCRS LFs in the $r$ band. This effect might be due to the bias against low surface brightness galaxies which affects the LCRS, and tends to exclude late-type galaxies. As a result, the low and high [OII]emission galaxy classes in the LCRS may be shifted towards earlier types. Comparison of LFs among different filters must however be taken with caution.

In the Norris survey (Small et al. 1997), shown in the lower right panel of Fig. 6, the 2 intrinsic LFs are also estimated using the strength of the [OII] $\lambda 3727$ emission line. Although a $\sim 0.7^{\mathrm{mag}}$ dimming of $M^{*}$ is observed for galaxies with $E W[\mathrm{OII}]>10 \AA$ compared to those with $E W[\mathrm{OII}]>$ $10 \AA$, no change in the slope $\alpha$ is observed between the 2 subsamples, probably due to poor statistics (see the large error bars). Although the Norris survey reaches $z_{\max } \simeq 0.5$, here I only consider the LFs for the sub-samples with $0<z \leq 0.2$, as the slope $\alpha$ for the $0.2<z \leq 0.5$ sub-samples is poorly determined (they only include galaxies brighter than $M(B) \lesssim-19$ ). The Norris LFs could be improved by extending the [OII]-line sub-samples to the full redshift range $0<z \leq 0.5$, thus doubling the number of galaxies per sub-sample (see Small et al. 1997). 
In contrast to the ESP and Norris surveys, the spectral classifications for the 2dFGRS1 and 2dFGRS2, whose LFs are also shown in the lower-right panel of Fig. 6, are based on a PCA, and use the projections onto the first 2 principal components (after exclusion of the mean spectrum). The 2dFGRS1 is separated into 5 types, which I estimate to correspond to morphological types E/S0, Sab, Sbc, Scd, and Sdm/Im resp. (see Fig. 8 of Folkes et al. 1999). The 2dFGRS2 is divided into 4 types; from Fig. 4 of Madgwick et al. (2002), I estimate that they correspond to morphological types $\mathrm{E} / \mathrm{S} 0 / \mathrm{Sa}, \mathrm{Sa} / \mathrm{Sb} / \mathrm{Scd}, \mathrm{Sb} / \mathrm{Scd}$, and $\mathrm{Scd} / \mathrm{Sm} / \mathrm{Im}$ respectively ${ }^{4}$.

The usual systematic steepening of the intrinsic LFs for later type galaxies is observed in both the 2dFGRS1 and 2dFGRS2 samples, and the 2 samples describe consistent intervals of $\alpha$ (within the error bars). Both samples also show a dimming in $M^{*}$ between the LFs for the earliest and the latest class (by $\sim 0.6^{\mathrm{mag}}$ for the $2 \mathrm{dFGRS1}$, and $\sim 0.4^{\mathrm{mag}}$ for the 2dFGRS2). Nonetheless, both the 2dFGRS1 and 2dFGRS2 fail to detect the Gaussian shape of the intrinsic LFs for E, S0 and Sa galaxies. Because the 2dFGRS1 and 2dFGRS2 samples reach absolute magnitudes as faint as $M(B) \simeq-16$ and $M(B) \simeq-14$ resp., both surveys should a priori detect the fall-off for the E, S0 and Spiral intrinsic LFs at faint magnitude (Jerjen \& Tammann 1997; see also Fig. 1 above). A dip at $-17.5 \lessgtr M\left(b_{\mathrm{J}}\right) \lessgtr-16.5$ is actually visible in the Type-1 LF for the 2dFGRS1 (see Fig. 11 of Folkes et al. 1999), and calls for confirmation with a larger sample. Although the 2dFGRS2 sample is $~ 20$ times larger than the 2dFGRS1, the 2dFGRS2 Type-1 LF only shows a weak minimum at $M\left(b_{\mathrm{J}}\right) \sim-16.0$. The different behavior between the 2 samples is probably due to the different definition of the spectral types.

Note that the 2dFGRS1 and 2dFGRS2 samples are separated into 1 and 2 more spectral classes resp. than the ESS and $\mathrm{CNOC} 2$; as a result, one would expect that their respective Type-1 samples show an even lower degree of morphological type mixing than in the ESS and CNOC2. The situation may however be opposite. As mentioned above for the LCRS, multi-fiber spectroscopy results in systematic color biases due to the small circular apertures, and in large random errors due to the inaccurate flux-calibration of the fibers and their inaccurate positioning onto the objects. Moreover, the design of the corrector lens of the $2 \mathrm{dF}$ multi-fiber spectrograph causes a chromatic displacement of different components of a given spectrum (Madgwick et al. 2002). The PCA of the 2dFGRS1 uses the flux-calibrated spectra using an average response curve of the instrument; this curve shows wavelength-dependent variations as large as $\sim 20 \%$ (Folkes et al. 1999), and does not account for the fiber-to-fiber and time variations, which cause additional dispersion in the flux-calibration. The 2dFGRS1 Type-1 classes may therefore be contaminated by galaxies with later spectral-types. As these have a nearly flat faint-end slope, the contamination tends to erase the Gaussian behavior of the $\mathrm{E}$ and S0 included in this class. In the 2dFGRS2, the Type-1 class contains predominantly E, S0 and Sa galaxies (see Fig. 4

\footnotetext{
${ }^{4}$ Although Kennicutt (1992a) galaxies with types later than Scd are not represented by Madgwick et al. 2002, I assume that these objects would be included in the latest class.
}

of Madgwick et al. 2002), which also have Gaussian LFs (see Sect. 2). The incompatibility of the 2dFGRS2 Type-1 LF (see Fig. 11 of Madgwick et al. 2002) with a Gaussian LF suggests that this sample is also affected by contamination among the spectral types.

The intrinsic LFs estimated from the commissioning data of the SDSS and based on rest-frame colors (Blanton et al. 2001) do not appear to be affected by these effects. Because Blanton et al. (2001) do not provide the Schechter parameters fitted to these LFs, they are not plotted in Fig. 4. From visual inspection of Fig. 14 of Blanton et al. (2001), the LFs in the $r^{*}$ band for the rest-frame color intervals $0.74<g^{*}-r^{*}<0.90$ and $0.58<$ $g^{*}-r^{*}<0.74$, which correspond to morphological types E, and S0/Sa galaxies resp. (see Fukugita et al. 1995) show a clear fall-off at faint magnitudes, in the intervals $-20.0 \lesssim M\left(r^{*}\right) \lesssim$ -18.3 , and $-19.0 \lesssim M\left(r^{*}\right) \lessgtr-16.7$ respectively. I suggest that the SDSS LFs are able to detect the bounded behavior at faint magnitude for the giant galaxies (E, S0, and Spiral) because these LFs are based on rest-frame colors and not on spectral classification. As the SDSS also uses multi-fiber spectroscopy, a spectral classification based on these data would likely be affected by aperture bias and calibration errors.

The 2dFGRS1 and 2dFGRS2 surveys show the same effect as observed for the ESS intermediate-type LF: nearly flat slopes are measured for the 2dFGRS1 Type- 2 LF ( $\alpha=-0.86$; this spectral class corresponds to morphological type $\mathrm{Sab}$ ) and Type-3 LF ( $\alpha=-0.99$; corresponding to type Sbc), and for the 2dFGRS2 Type-2 LF ( $\alpha=-0.99$; corresponding to types $\mathrm{Sa} / \mathrm{Sb} / \mathrm{Scd}$ ). In the ESS, these flat slopes are reconciled with the Gaussian shapes of the intrinsic LF for Spiral galaxies by adding a contribution from dwarf spheroidal galaxies (see Fig. 11 in de Lapparent et al. 2003b), which is justified by the bluer colors of the $\mathrm{dSph}$ galaxies as compared to their giant analogs (E and S0 type). I propose a similar interpretation of the flat slopes of the intermediate-type LFs for the 2dFGRS1 and 2dFGRS2 surveys; it could also apply to the SDSS LF for rest-frame colors $0.42<g^{*}-r^{*}<0.58$ (corresponding to Sbc/Scd galaxies; see Fukugita et al. 1995), which has a flat faint-end slope (see Fig. 14 in Blanton et al. 2001). This interpretation is at variance with that of Kochanek et al. (2001), who show that in redshift surveys based on multi-fiber spectroscopy, the mix of the various morphological classes yields a false artificial steep slope for the Spiral galaxies: the interpretation of Kochanek et al. (2001) ignores the Gaussian behavior of the Spiral intrinsic LF.

Note that the SDSS LF for the earliest class $(0.74<$ $g^{*}-r^{*}<0.90$ ) shows an upturn at $M\left(r^{*}\right) \gtrsim-18.0$ (Blanton et al. 2001). I already mentioned that the Type-1 LFs for the 2dFGRS1 and 2dFGRS2 show an upturn at $M\left(b_{\mathrm{J}}\right) \gtrsim-17.0$ and $M\left(b_{\mathrm{J}}\right) \gtrsim-16.0$ respectively. These upturns could be explained by a population of red dSph galaxies, as detected in the Coma cluster (see Andreon \& Cuillandre 2002). However, at these faint magnitudes, the signal in the early-type LFs for the SDSS and 2dF samples is of low significance, and calls for caution in its interpretation; if such a population exists, it appears of lower density than the population of bluer dSph galaxies which presumably flattens the intermediate-type LFs in the 
ESS (de Lapparent et al. 2003b) and could also play the same role in the SDSS, 2dFGRS1 and 2dFGRS2.

The slopes $\alpha=-1.21$ and $\alpha=-1.24$ for the Type- $4 \mathrm{LF}$ in the 2dFGRS1 (corresponding to morphological types Scd) and the Type-3 LF in the 2dFGRS2 (corresponding to types $\mathrm{Sb} / \mathrm{Scd}$ ) resp., are also both symptomatic of type mixing, as galaxies with these morphological types are expected to have Gaussian LFs (see Jerjen \& Tammann 1997 and Fig. 1). Therefore, it is likely that these spectral classes are contaminated by the later type galaxies $(\mathrm{Sm} / \mathrm{Im})$. As shown for the ESS late-type LF (de Lapparent et al. 2003b), combination of a Gaussian LF for the giant galaxies and a steep Schechter LF for the dwarf galaxies yields a Schechter LF with an intermediate faint-end slope. Indeed, steep faint-end slopes $\alpha=-1.73$ and $\alpha=-1.50$ are measured for the Type-5 LF in the $2 \mathrm{dF}$ GRS1 (corresponding to morphological types $\mathrm{Sdm} / \mathrm{Im}$ ), and the Type-4 LF in the 2dFGRS2 (corresponding to Scd/Sm/Im) respectively. The former is in good agreement with the values obtained for the same types in the nearby redshift surveys CfA2S and SSRS2, based on morphological classification (see the upper-left panel of Fig. 6, and Table 4; these surveys are described in Sect. 3.3.4 below); the flatter slope for the Type-4 LF in the 2dFGRS2 may again be symptomatic of type mixing.

\subsection{3. $B$ luminosity functions at redshifts $0.02-0.15$}

The upper-right panel of Fig. 6 shows the $M^{*}$ and $\alpha$ parameters for the intrinsic LFs measured from redshift surveys with $0.02 \lesssim z_{\max } \lesssim 0.15$ : the Stromlo-APM survey (Loveday et al. 1992, 1999, denoted SAPM), the original Durham-AngloAustralian-Telescope Redshift Survey (Efstathiou et al. 1988, denoted DARS1), and its improved multi-color measurements (Metcalfe et al. 1998, denoted DARS2); and the Autofib survey (Heyl et al. 1997). The photometric catalogues on which are based all the mentioned redshift surveys are obtained by digitization of photographic plates from the UK Schmidt telescope (Efstathiou et al. 1988; Collins et al. 1989; Maddox et al. 1990b).

Again, I convert the values of $M^{*}$ measured as $b_{\mathrm{J}}$ magnitudes into the Johnson $B$ band using the $B-b_{\mathrm{J}}=0.28(B-V)$ color equation determined by Blair \& Gilmore (1982, see also Norberg et al. 2002), complemented by the $B-V$ colors estimated by Fukugita et al. (1995, Table 3a): for the DARS1 and the 2-class SAPM (Loveday et al. 1992), I use the average $B-V$ color 0.905 over listed types $\mathrm{E}$ and $\mathrm{S} 0$, and 0.57 for listed type Sbc; for the types based on the $E W(\mathrm{H} \alpha)$ in the SAPM (Loveday et al. 1999), I use the average $B-V$ color 0.905 over listed types $\mathrm{E}$ and $\mathrm{S} 0$, the average $B-V$ color 0.675 over listed types Sab and Sbc, and the average $B-V$ color 0.385 over listed types Scd and Im; for the 6 Autofib types, I use the $B-V$ colors $0.96,0.85,0.78,0.57,0.50,0.27$, for listed types $\mathrm{E}$, $\mathrm{S} 0$, Sab, Sbc, Scd, Im respectively. The resulting $B-b_{\mathrm{J}}$ colors are 0.25 for the average between listed types E and S0, and 0.16 for listed type Sbc, assigned to the DARS1 and the 2-class SAPM (Loveday et al. 1992); 0.25 for the average between listed types E and S0, 0.19 for the average between listed types Sab and Sbc, and 0.11 for the average between listed types Scd and Im, which are assigned to the SAPM classes based on the $E W(\mathrm{H} \alpha)$ (Loveday et al. 1999); 0.27, 0.24, 0.22, 0.16, 0.14, 0.08 for listed types E, S0, Sab, Sbc, Scd, Im resp., which are assigned to the Autofib types.

Although the Autofib survey probes the galaxy distribution to $z \sim 0.75$, the most reliable constraints on the faint-end slope $\alpha$ of the intrinsic LFs are obtained for $0.02<z \leq 0.15$ (see Figs. 15, 17 and 20 of Heyl et al. 1997); the LFs in the intervals $0.15<z \leq 0.35$ and $0.35<z \leq 0.75$ are used by the authors to constrain the evolution in each LF. Here, I thus use the Schechter parameters calculated at $z=0.1$ from the parameters given in Table 2 of Heyl et al. (1997); the resulting values are listed in Table 4 (note that no uncertainties in the Autofib LFs are provided by Heyl et al. 1997).

I first describe the intrinsic LFs measured by the Autofib survey (Heyl et al. 1997). These LFs describe an even narrower interval of faint-end slope than the 2dFGRS1 and 2dFGRS2: $-1.36 \leq \alpha \leq-0.99$. The flat slope $(\alpha \sim-1.0)$ measured for the 3 classes red-E, blue-E, and Sab galaxies means that the Autofib survey fails to detect the bounded behavior of the early-type LF at faint magnitudes, which is characterized by $-0.4 \lesssim \alpha \lesssim 0.2$ in the other surveys of the graph. This may also be the result of a contamination among the galaxy classes. The Autofib survey uses a spectral classification method based on cross-correlation with a set of templates. The cross-correlation technique is efficient for measuring redshifts of absorption-line spectra, as the signal which builds the cross-correlation peak in a given spectrum is contributed to by all the absorption lines in that spectrum (Tonry \& Davis 1979). In this approach, the continuum must be subtracted and low-pass filtered, which is at marked variance with the fact that in a spectral classification, the dominant signal originates from the shape of the continuum of the spectra (Galaz \& de Lapparent 1998). Although there is a correlation between the absorption line pattern and the continuum of a spectrum, the absorption lines are sensitive to signal-to-noise, to the efficiency of the sky subtraction, and to the contamination by $\mathrm{OH}$ sky emission and cosmic rays. When used as a spectral classification, the cross-correlation technique therefore implies some dispersion due to the various mentioned effects.

Here I provide direct evidence that the classification based on spectrum cross-correlation is responsible for type mixing among the various classes: the redshifts for the ESS absorptionline spectra were actually measured by cross-correlation with average Kennicutt (1992a) template spectra (Bellanger et al. 1995; see details on templates in Sect. 2.2 of de Lapparent et al. 2003b), thus providing as a byproduct the cross-correlation types. I am then able to compare the ESS cross-correlations types with the PCA spectral types obtained for the same galaxies. Figure 7 shows the 3 histograms of the ESS galaxies with $R_{\mathrm{c}} \leq 20.5$ classified as E/S0 (211 galaxies), $\mathrm{Sa} / \mathrm{Sb}$ (88 galaxies), Sc/Ir (299 galaxies) by the cross-correlation technique, as a function of the spectral type $\delta$. The 2 vertical lines are the corresponding $\delta$ boundaries between the 3 spectral classes corresponding to morphological types $\mathrm{E} / \mathrm{S} 0, \mathrm{Sa} / \mathrm{Sb}, \mathrm{Sc} / \mathrm{Ir}$, and estimated by projection of the Kennicutt spectra onto the ESS spectral sequence (see Fig. 2b of de Lapparent et al. 2003b). Figure 7 shows that the ESS E/S0 cross-correlation class (solid 


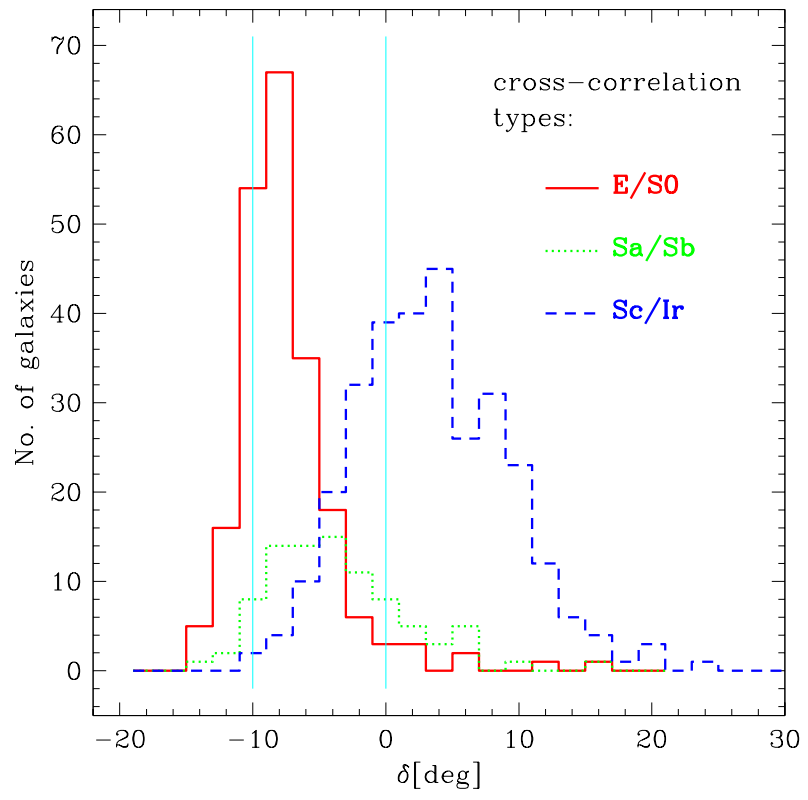

Fig. 7. Distribution of the ESS "cross-correlation" types as a function of spectral type $\delta$. The 3 "cross-correlation" classes are obtained by cross-correlating with average spectra of galaxies with morphological types E, S0, Sa, Sb, Sc, and Ir ( from Kennicutt 1992a), and subsequent grouping of the cross-correlation types in the 3 classes defined by E/S0, Sa/Sb, Sc/Ir. The vertical lines mark the corresponding boundaries in $\delta$ for these 3 morphological classes (de Lapparent et al. 2003b).

line histogram) widely overlaps with galaxies of $\mathrm{Sa} / \mathrm{Sb}$ spectral type, which acts as a contamination of the E/S0 LF measured from cross-correlation types; moreover, the $\mathrm{Sa} / \mathrm{Sb}$ crosscorrelation class (dotted line histogram) describes a $\delta$ interval which is nearly fully included into that described by the E/SO histogram. This effect could explain how the Autofib survey fails to measure the early-type intrinsic LF, and why its red-E, blue-E, and Sab LFs have similar faint-end slopes. In a similar fashion, Fig. 7 shows that spectral types $\mathrm{Sa} / \mathrm{Sb}$ significantly contaminate the cross-correlation types $\mathrm{Sc} / \mathrm{Ir}$ : this could provide an interpretation of the similar shape parameters $M^{*}$ and $\alpha$ measured for the Sbc, Scd and Sdm-Starburst LFs in the Autofib survey. This analysis shows that a more robust spectral classification is obtained by a PCA classification as used for the ESS, or by least-square fit of the SEDs to spectral libraries, as used in the CNOC2 survey, rather than by cross-correlation with templates.

Note that the suspected presence of type mixing in the Autofib spectral classes complicates the interpretation of the evolution in these LFs, parameterized in Table 2 of Heyl et al. (1997). When calculating the Autofib Schechter parameters for $z=0.5$ (using Table 2 in Heyl et al. 1997), I derive a wider interval of faint-end slope $-1.75 \leq \alpha \leq-0.40$, with the following individual values: -0.40 for Red-E, -0.45 for Blue-E, -1.99 for Sab, -1.28 for Sbc, -1.54 for Scd, -1.75 for SdmStarburst. Except for the Sab galaxies, which appear to have an anomalously large evolution rate in $\alpha$, the values of $\alpha$ for the other classes are remarkably close to those measured from the 5 spectral-class $2 \mathrm{dFGRS} 1 \mathrm{LFs}$; the range of $M^{*}$ values described by the Autofib at $z=0.5$ are also comparable to those for the 2dFGRS1, except for the Autofib Sab galaxies. Because the $2 \mathrm{dFGRS} 1$ has $z_{\max } \simeq 0.15$, this comparison casts some doubts onto the detected evolution in the Autofib intrinsic LFs (Heyl et al. 1997).

In contrast with the Autofib survey and those with $0.15 \lesssim$ $z_{\max } \lesssim 0.20$ (lower-right panel of Fig. 4), for which $\alpha$ for all galaxy types is steeper than $\sim-0.5$, the SAPM, DARS1, and DARS2 surveys taken together describe the same range in $\alpha$ as the deep surveys (with $z_{\max } \sim 0.6$; lower-left panel of Fig. 4), with some LFs having values of $\alpha$ in the interval $-0.5 \lesssim \alpha \lesssim 0.5$. For the 3 surveys, $\alpha$ steepens for later types. However, the value of $\alpha$ varies by at least 0.5 from survey to survey for a given class (see Table 4 ). As for the CNOC1 and CFRS $B$ LFs, based on 2 galaxy classes, $M^{*}$ is brighter for later types in both the DARS1 and DARS2. Examination of the corresponding curves (Efstathiou et al. 1988; Metcalfe et al. 1998) confirms that again, this is due in part to the correlation between $M^{*}$ and $\alpha$ : the bright-end of the late-type LFs is fainter than for the early-type LF by $\sim 0.1-0.2^{\mathrm{mag}}$ for the DARS1, and by $\sim 0.5^{\mathrm{mag}}$ for the DARS2. As for the CNOC1 and CFRS $B$ LFs, the small change in the bright-end of the LFs, characterized by an "inverted" $M^{*}$ behavior, appears to be caused by the use of only 2 classes, which fails in separating the blue low luminosity galaxies from the luminous Spiral galaxies. The late-type class in the DARS1, which contains Spiral to Irregular (denoted Irr) galaxies, might also be deficient in blue low luminosity galaxies, which contributes to the dimming of $M^{*}$ : these galaxies have a lower surface brightness, and are difficult to detect and classify visually. The weaker brightening in $M^{*}$ for the Sp-Irr galaxies in the DARS2, which marks a larger dimming of the LF bright-end for later types, may result from the separation of the 2 classes using rest-frame color instead of the morphological types used in the DARS1, and from the use of aperture magnitudes for the DARS2, in replacement of the isophotal magnitudes in the DARS1.

Although the SAPM LFs for E-S0 and Sp-Irr morphological types resp. (Loveday et al. 1992) do detect a $0.25^{\mathrm{mag}} \mathrm{dim}-$ ming of $M^{*}$ for the later class, the shift between the bright-ends of the 2 LFs is $\sim 0.2^{\mathrm{mag}}$, as small as for the DARS1 survey: here, $M^{*}$ does not display an "inverted" behavior because the Sp-Irr LF has $\alpha=-0.8$, which implies that $M^{*}$ reflects the location of the bright-end (see Sect. 3.1.2). The fact that the SAPM LF for Sp-Irr galaxies fails to detect the expected steep slope for the Irr galaxies, may be due in part to the use of only 2 sub-samples, and also to incompleteness: the total SAPM spectroscopic sample amounts to 1658 galaxies, among which 1310 were classified as E, S0, Spiral or Irr from visual examination of the photographic plates; one may suspect that the 348 unclassified galaxies contain predominantly low surface brightness objects, as these are more difficult to classify visually. Among the low surface brightness galaxies are the late-type low luminosity galaxies (Sd, Sm, Irr), which are the major contributors to the steep faint-end slope of the late-type LF. The SAPM intrinsic LF for the galaxies with strong $\mathrm{H} \alpha$ emission line (Loveday et al. 1999, sub-sample with $E W(\mathrm{H} \alpha) \geq 15 \AA$ in 
Table 4$)^{5}$, does have a steep faint-end slope $\alpha=-1.28 \pm 0.30$; this improvement may be due to the significant sample of galaxies in this class which are not morphologically classified (see notes of Table 4), and as mentioned above, might be preferentially Sd, Sm and Irr galaxies, those contributing to the steep faint-end slope.

A remarkable result is that the SAPM succeeds in detecting the sharp fall-off at faint magnitudes of the LF for morphological types E-S0, which is characterized by $\alpha=0.20 \pm 0.35$ (note that the above mentioned incompleteness would not bias this result). From visual examination, it appears that a Gaussian LF might actually provide a good fit to the SAPM E-SO LF. This confirms the reliability of the APM morphological classification for the E-S0 galaxies, despite some scatter, as tested by Naim et al. (1995). I emphasize that among the redshift surveys to intermediate redshifts $\left(0.02 \leq z_{\max } \leq 0.2\right.$, shown in the 2 upper panels and in the lower-right panel of Fig. 6), the SAPM it is the only survey which has such a large value of $\alpha$ for the early-type LF, in agreement at the 1- $\sigma$ level with the values of the $B$ early-type LFs for the CFRS ( $\alpha=0.00 \pm 0.20)$, CADIS $(\alpha=0.18 \pm 0.22)$, CNOC2 $(\alpha=0.08 \pm 0.14)$, and ESS $(\alpha=-0.24 \pm 0.33)$.

In contrast, the nearly flat slopes $\alpha=-0.75 \pm 0.28$, and $\alpha=-0.72 \pm 0.29$ of the LFs for the SAPM galaxies with low and intermediate $E W(\mathrm{H} \alpha)$ resp., are symptomatic of type mixing, as in the ESP LF of low [OII]-emission galaxies. It is also noticeable that the SAPM LFs based on the equivalent width $\mathrm{H} \alpha$ are the only emission-line LFs which show simultaneously 2 properties of the local intrinsic LFs: (i) a significant dimming in $M^{*}$ between the early-type and late-type galaxies (namely $\simeq 0.5^{\mathrm{mag}}$ ); (ii) a steep faint-end slope for the late-type galaxies; these 2 properties are not observed simultaneously in either the LCRS $r$ LFs (see Sect. 3.2), the ESP or the Norris $B$ LFs. This may be due to the joint effect of using 3 classes together with the $\mathrm{H} \alpha$ line, whereas the other surveys (LCRS, ESP, Norris) use only 2 classes and the [OII] line.

\subsubsection{B luminosity functions at redshifts below 0.03}

Finally, the upper-left panel of Fig. 6 shows the intrinsic LFs for the following nearby redshift survey (with $z_{\max } \lesssim 0.03$ ): the Nearby Optical Galaxy survey (Marinoni et al. 1999, denoted NOG); the Center for Astrophysics Redshift Survey to $B_{\mathrm{Zw}} \leq 14.5$ (Davis et al. 1982, CfA1), complemented by the first 2 slices of the extension to $B_{\mathrm{Zw}} \leq 15.5$ (Marzke et al. 1994a, the combination of the 2 surveys is denoted CfA2S); the Southern Sky Redshift Survey (Marzke et al. 1998, denoted SSRS2). For the CfA2S and SSRS2, conversion of $M^{*}$ measured as Zwicky magnitude $B_{\mathrm{Zw}}$ into a Johnson $B$ magnitude is based on $b_{\mathrm{J}}=B_{\mathrm{Zw}}-0.35$ from Gaztañaga \& Dalton (2000), on the $B-b_{\mathrm{J}}=0.28(B-V)$ color equation determined by Blair \& Gilmore (1982, see also Norberg et al. 2002), and on the $B-V$ colors calculated by Fukugita et al. (1995, Table 3a): for the 5 CfA2S morphological types, I use the $B-V$ colors

\footnotetext{
5 A classification based on the $E W[\mathrm{OII}]$ is also obtained by the authors, and yields similar results. I however favor the results based on $\mathrm{H} \alpha$ as this line provides a better indicator of the current star formation rate (Kennicutt 1992b; Charlot \& Longhetti 2001).
}

$0.96,0.85,0.78,0.50,0.27,0.04$, for listed types E, S0, Sab, Scd, Im resp.; for the 3 SSRS2 morphological types, I use the average $B-V$ color 0.905 over listed types E and S0, and 0.57 , 0.27 for listed types $\mathrm{Sbc}$, Im respectively. The resulting $B-b_{\mathrm{J}}$ colors are $0.27,0.24,0.22,0.14,0.08$ for listed types E, S0, $\mathrm{Sab}, \mathrm{Scd}$, Im resp., assigned to the CfA2S; 0.25 for the average between listed types E and S0, and 0.16, 0.08 for listed types Sbc, Im resp., assigned to the SSRS2.

The NOG, CfA2S and SSRS2 redshift surveys are all based on galaxy catalogues extracted from photographic plates (Zwicky et al. 1968; Nilson 1973; Laubert et al. 1981; Lasker et al. 1990), and the intrinsic LFs are based on the morphological types in the revised Hubble classification scheme (see de Vaucouleurs et al. 1991). The morphological classes corresponding to the plotted points are indicated in Table 4. The variations in $\left[M^{*}, \alpha\right]$ for the 3 surveys resembles those for the SDSS-Morph survey in the $R_{\mathrm{c}}$ band: only the LFs for the latest class, corresponding to Sm-Im galaxies in the CfA2S and NOG surveys, and to Irr-Pec galaxies in the SSRS2, show a clear steepening of $\alpha$, whereas the LFs for earlier types have $-1.0 \lesssim \alpha \lesssim-0.5$. The steep faint-end slopes measured for the Sm-Im LFs in the NOG $(\alpha=-2.41 \pm 0.28)$ and the CfA2S $(\alpha=-1.87 \pm 0.15)$, and the Irr-Pec LF in the SSRS2 $(\alpha=-1.81 \pm 0.24)$ suggest that the field LF for Sm-Im galaxies might be on the average as steep or stepper than in the Centaurus cluster, for which $\alpha=-1.35$ (see Table 1 in Sect. 2). However, in the 3 samples, the latest class does not show the dimming in $M^{*}$ detected in the ESS, CADIS and COMBO-17 B LFs (see lower-left panel of Fig. 6), and in the SDSS-Morph LFs converted into the $R_{\mathrm{c}}$ filter (see left panel of Fig. 4), which is caused by a dominating population of dI galaxies in these classes: there is no change in $M^{*}(B)$ from the Sc-Sd to the Sm-Im LF in the CfA2S, a $0.73^{\text {mag }}$ brightening from the Sc-Sd to the Sm-Im LF in the NOG, and a $0.39^{\text {mag }}$ brightening from the Spiral to the Irr-Pec LF in the SSRS2. The magnitude difference between the peak magnitude $M_{0}$ of the Sc Gaussian LF and the Schechter $M^{*}$ for the dI galaxies is $1.7^{\mathrm{mag}}, 1.5^{\mathrm{mag}}$ and $1.4^{\mathrm{mag}}$ in the $R_{\mathrm{c}}, V$ and $B$ bands resp. (see Table 1), and is therefore expected to be detectable in all 3 filters. The absence of a shift towards fainter values of $M^{*}$ between the Sc-Sd/Spiral LFs and the Sm-Im/Irr-Pec LFs in the NOG, CfA2S and SSRS2 surveys suggests that the Sm-Im/IrrPec classes in these surveys might be contaminated by galaxies of earlier morphological type, and thus higher luminosity.

Although the NOG, CfA2S and SSRS2 probe the LFs to $M(B) \simeq-14.5$, nearly $2^{\text {mag }}$ fainter than in the SDSS-Morph, neither the CfA2S nor the SSRS2 detect the Gaussian shape of the intrinsic LFs measured for local E and S0 galaxies, which would be characterized by a fall-off the LFs by a factor 10 or more at $M\left(R_{\mathrm{c}}\right) \gtrsim-17$ (see Fig. 1 ), that is $M(B) \gtrsim-16$ using the $B-R_{\mathrm{c}}$ color of an Sbc galaxy (Fukugita et al. 1995): both the CfA2S and the SSRS2 measure nearly flat slopes for the E and S0 LFs out to $M(B) \simeq-14.5$, similarly to the SDSS-Morph LFs in the $R_{\mathrm{c}}$ band. The CfA2S and SSRS2 E-S0 sub-samples might therefore contain a contribution from dSph galaxies, in a similar fashion as the E-S0 LF in the SDSS-Morph sample (Nakamura et al. 2003, see Sect. 3.2). Only the NOG obtains a bounded behavior at faint magnitudes for the E galaxy LF, with 
$\alpha=-0.47 \pm 0.22$. A faint-end bounded LF is also measured for the NOG $\mathrm{Sa}-\mathrm{Sb}$ galaxies.

At last, the LFs for giant Spiral galaxies $(\mathrm{Sa}, \mathrm{Sb}, \mathrm{Sc})$ in the CfA2S and SSRS2, and for the S0 and Sc-Sd galaxies in the NOG all have nearly flat slopes. These sub-samples might also contain a contribution from dwarf galaxies, in a similar fashion as the ESO-Sculptor intermediate-type LF (de Lapparent et al. 2003b). The NOG, CfA2S and SSRS2 morphological classifications are also likely to be subject to some amount of type mixing, due to the dispersion in visual classification techniques (Lahav et al. 1995).

\subsection{I band}

I found no measurement of intrinsic LFs in the $I$ band. Despite the $I$ selection of the CFRS (Lilly et al. 1995) and CADIS samples (Fried et al. 2001), only $B$ intrinsic LFs are measured for these samples.

\subsection{SDSS and Durham general luminosity functions}

For comparison of the intrinsic LFs with the unique measurement of the "general" LF in a given band, I plot in Figs. 2, 4 and 6 the Schechter parameters of the general LFs for: the Sloan Digital Sky Survey (Blanton et al. 2003, denoted SDSS), which provides $u^{*} g^{*} r^{*} i^{*} z^{*}$ measurements in the SDSS filters (Fukugita et al. 1996) blueshifted by $z=0.1$; and the Durham-Anglo-Australian-Telescope Redshift Survey $U B V R_{\mathrm{c}} I_{\mathrm{c}}$ re-measurements (Metcalfe et al. 1998, denoted DARS2). The SDSS and DARS2 surveys provide the only 5-band multi-color measurements of the general LF in the optical. They may therefore serve as reference for comparison among the different filters. Because the DARS2 LF is split into 2 color-based intrinsic LFs in the $B$ band, I do not plot its general LF in this filter. I show instead the $B$ general LF measured from the Durham-UK-Schmidt-Telescope Redshift Survey (Ratcliffe et al. 1998, denoted DUKST).

The SDSS general LFs in the $u^{*} g^{*} r^{*} i^{*}$ bands (Fukugita et al. 1996) are converted into the $U B V R_{\mathrm{c}} I_{\mathrm{c}}$ bands using the following transformations, based on the colors of an Sbc galaxy (Fukugita et al. 1995, Tables 3a and 3m; here we assume that the color changes occurring when blueshifting the SDSS filters by $z=0.1$ are negligible):

$$
\begin{array}{ll}
M^{*}(U)=M^{*}\left(u^{*}\right)-0.82 & ; \alpha(U)=\alpha\left(u^{*}\right) ; \\
M^{*}(B)=M^{*}\left(g^{*}\right)+0.34 & ; \alpha(B)=\frac{\alpha\left(u^{*}\right)+\alpha\left(g^{*}\right)}{2} ; \\
M^{*}(V)=M^{*}\left(g^{*}\right)-0.23 & ; \alpha(V)=\alpha\left(g^{*}\right) ; \\
M^{*}\left(R_{\mathrm{c}}\right)=M^{*}\left(r^{*}\right)-0.23 & ; \alpha\left(R_{\mathrm{c}}\right)=\alpha\left(r^{*}\right) ; \\
M^{*}\left(I_{\mathrm{c}}\right)=M^{*}\left(i^{*}\right)-0.51 & ; \alpha\left(I_{\mathrm{c}}\right)=\alpha\left(i^{*}\right) .
\end{array}
$$

Note that the uncertainty in $\alpha(B)$ is estimated as $\sqrt{\sigma_{\alpha}\left(u^{*}\right)^{2}+\sigma_{\alpha}\left(g^{*}\right)^{2}} / 2$, as implied by Eq. (4) above. The $M^{*}$ value in the $b_{\mathrm{J}}$ band measured for the DUKST is converted into the $B$ band using the intermediate $B-V=0.57$ intermediate color for listed type Sbc in Fukugita et al. (1995), and the $B-b_{\mathrm{J}}=0.28(B-V)$ color equation determined by
Blair \& Gilmore (1982, see also Norberg et al. 2002), yielding $B-b_{\mathrm{J}}=0.16$. The resulting Schechter parameters for the SDSS, DARS2, and DUKST are listed in Tables 2 to 4 (the parameters for the $I$ LFs are listed at the end of Table 3 ).

Figures 2, 4 and 6 show that when compared with the Schechter parameters for the intrinsic LFs in the same filter, the general LFs for the DARS2 (and the DUKST) have values of $M^{*}$ comparable or brighter than the values among the intrinsic LFs. This is in agreement with the expectation that in a general LF, $M^{*}$ is principally determined by the most luminous galaxies in the sample. In contrast, the SDSS LFs have values of $M^{*} \sim 1^{\mathrm{mag}}$ fainter than in the DARS2 (in $U, V, R_{\mathrm{c}}$ ), thus lying at the median or faintest values of $M^{*}$ measured for the intrinsic LFs at similar redshifts. This difference may be due to the fact that redshift evolution is accounted for in the derivation of the SDSS LFs, whereas this is not the case for the other surveys with $z_{\max } \lesssim 0.2$. Moreover, the general LFs for the SDSS, DARS2, and DUKST have flat or slightly steeper slopes $(-1.04 \leq \alpha \leq-0.90$ for the SDSS and DUKST; for the DARS2, $\alpha=-1.20$ is fixed to the value measured in the $B$ band), whatever the range of $\alpha$ measured for the intrinsic LFs of the various surveys in the corresponding filter. I have shown above how type mixing inevitably results in a nearly flat faintend slope. This also applies to the general LF.

The general LF over a complete region of the Universe, as sampled in systematic redshift surveys, thus provides no direct indication on the Gaussian nature of the LFs for the giant galaxies, and on a steep faint-end slope for the dwarf galaxies. Given the variety of intrinsic LFs described in Sect. 2, it is a priori surprising that the mixing of all galaxy types in redshift surveys results in a general LF which is well fit by a Schechter function. In local surveys of galaxy concentrations, the contributions from giant and dwarf populations are both detected, and their signatures are an exponential fall-off at bright magnitudes, and a steep power-law behavior a faint magnitudes resp., with a plateau or a knee in the intermediate regime (see for example Trentham \& Tully 2002). Similar behaviors are detected in general LFs for clusters of galaxies at higher redshift (Driver et al. 1994; Wilson et al. 1997; Trentham 1998; Garilli et al. 1999; Durret et al. 2000; Beijersbergen et al. 2002; Yagi et al. 2002; Mobasher et al. 2003). In systematic redshift surveys over a given region of the Universe, the contribution from galaxy concentrations is still present. It is however complemented by the contribution from the numerous field Spiral galaxies, existing in a larger proportion than in groups and clusters. It it likely that the field Spiral galaxies cause a significant increase in the general LF at intermediate magnitudes, thus "filling-in" the mentioned "plateau" region, and making the Schechter form an adequate description over more than 5 mag. Then, as suggested by Binggeli et al. (1988) and Ferguson \& Sandage (1991), the variations of $M^{*}$ and $\alpha$ in the general LF as a function of sample and filter may simply reflect the average proportions of the various galaxy types in the survey region.

\section{Conclusions and prospects}

I perform a detailed comparison of all the existing measurements of intrinsic LFs in the optical domain, derived from redshift surveys with effective depth $z \simeq 0.03$ to 0.6 and 
converted into the $U B V R_{\mathrm{c}} I_{\mathrm{c}}$ system wherever necessary. The shape of the various LFs is compared among the different surveys and galaxy classes, using the Schechter parameters $M^{*}$ and $\alpha$. In this comparison, I use as reference the intrinsic LFs per morphological type measured from local galaxy concentrations (Sandage et al. 1985; Jerjen \& Tammann 1997).

Each survey detects variations in the shape of the LF with galaxy type. However, the LFs for a given galaxy type widely vary from survey to survey. I interpret these differences in terms of the classification schemes for defining the galaxy classes (based on morphological types, spectral types, crosscorrelation types, colors, or equivalent width of emission lines), and show that they often induce some mixing of distinct morphological types, which in turn complicates the interpretation of the LFs.

The salient results which I emphasize or derive in the present analysis are:

- Spectral classification with accurate flux calibration and a minimum of 3 classes allows to observe both the Gaussian early-type LF (corresponding to E, SO, and sometimes Sa galaxies), and the dimming of the late-type LF (containing usually $\mathrm{Sc}, \mathrm{Sd} / \mathrm{Sm}$ and Irr galaxies) as illustrated by the CNOC2 (Lin et al. 1999) and ESO-Sculptor Survey (de Lapparent et al. 2003b).

- The nearby Center for Astrophysics Redshift Survey (Marzke et al. 1994a), the Southern Sky Redshift Survey (Marzke et al. 1998), and the deeper sample extracted from the SDSS Early Data Release (Nakamura et al. 2003), all based on visual morphological classification, detect a nearly flat faint-end slope for their earliest-type LFs, thus failing to detect the Gaussian E/S0 LF; moreover, these surveys fail to detect the dimming of the Sm-Im LF compared to the Sc-Sd LF.

- The Autofib (Heyl et al. 1997) and the 2 preliminary samples of the $2 \mathrm{dF}$ Galaxy Redshift Survey samples (Folkes et al. 1999; Madgwick et al. 2002, 2dFGRS), all based on spectral classification, also fail to detect the Gaussian LF for E/S0.

- Although the Autofib survey (Heyl et al. 1997) is based on slit spectroscopy, a fair amount of type mixing appears to bias the derived LFs, because of inaccuracies in the spectral classification which is based on cross-correlation with galaxy templates; the effect is demonstrated using the ESO-Sculptor Survey (de Lapparent et al. 2003b), for which both cross-correlation types and PCA (Principal Component Analysis) spectral types are available.

- The continuous variation in the Schechter faint-end slope of the spectral-type LFs measured in $b_{\mathrm{J}}$ for the 2 preliminary samples of the 2dFGRS (Folkes et al. 1999; Madgwick et al. 2002), and in the 6 spectral-type LFs measured in $R_{\mathrm{c}}$ for the Las Campanas Redshift Survey (Bromley et al. 1998) is interpreted as type mixing between the giant galaxies with Gaussian LFs and the dwarf galaxies with Schechter LFs: this partly results from the aperture and flux-calibration biases affecting redshift surveys obtained with multi-fiber spectrographs.
- When LFs are measured for 2 sub-samples separated by color (as in the Canada-France Redshift Survey, Lilly et al. 1995; the CNOC1 survey, Lin et al. 1997; and the Century Survey, Brown et al. 2001), or separated by the equivalent width of characteristic emission lines (as in the ESO Slice Project, Zucca et al. 1997; the Norris survey, Small et al. 1997; and the Stromlo-APM survey, Loveday et al. 1999; see also Lin et al. 1996) they are insufficient for estimation of the intrinsic LFs as they not only fail to separate the various populations of giant and dwarf galaxies, but they also mix giant galaxies of different morphological type; this effect is illustrated using the ESO-Sculptor Survey (de Lapparent et al. 2003b), for which PCA spectral types, colors and equivalent width of [OII] emission are available.

- Although the COMBO-17 LFs in the $B$ band (Wolf et al. 2003) are consistent with those from the comparable CADIS (Fried et al. 2001), and with those from the CNOC2 (Lin et al. 1999) and ESO-Sculptor (de Lapparent et al. 2003b) surveys, the COMBO-17 LFs converted into the $U$ and $R_{\mathrm{c}}$ bands shows significant differences with the CNOC2 and ESO-Sculptor for the intermediate spectral types corresponding to Spiral galaxies. This may result from the complex selection effects inherent to the use of medium-band photometry for redshift measurement in the COMBO-17 survey, and/or from its color transformations from the $r^{*}$ and $m_{280}$ bands into the $R_{\mathrm{c}}$ and $U$ bands resp. (Wolf 2002).

One conclusion which I draw from these various results is that the spectral classifications used in the CNOC2 (Lin et al. 1999) and ESO-Sculptor (de Lapparent et al. 2003b) surveys, both based on multi-slit spectroscopy, provide the least biases estimates of intrinsic LFs. The CADIS (Fried et al. 2001) and COMBO-17 (Wolf et al. 2003) surveys, based on photometric redshifts using medium-band filters, also provide consistent intrinsic LFs in the $B$ band with the CNOC2 and ESO-Sculptor. The 4 mentioned surveys are based on CCD photometry combined with a spectral classification with accurate flux calibration, which therefore appears, among the mentioned surveys, as the optimal combination for estimating the intrinsic LFs. The systematic effects affecting a spectral classification based on multi-fiber spectroscopy as in the 2dF Galaxy Redshift Survey (Folkes et al. 1999; Madgwick et al. 2002) cause type mixing among the various morphological classes which significantly biases the estimates of intrinsic LFs.

Surprisingly, spectral classification at $z \sim 0.5$ provides better estimates of the intrinsic LFs than the first generation of redshift surveys to $z \lesssim 0.03$ (the Nearby Optical Galaxy survey, Marinoni et al. 1999; the Center for Astrophysics Redshift Survey, Marzke et al. 1994a; and the Southern Sky Redshift Survey Marzke et al. 1998), although the latter surveys are based on direct morphological classification. The intrinsic LFs derived from the nearby surveys are likely to be biased in their magnitudes and morphological classification because of (i) the non-linear response of photographic plates, (ii) their narrow dynamic range, and (iii) the human estimation of the magnitudes and galaxy types (see Lahav et al. 1995). 
Visual morphological classification is indeed largely subjective, even from good quality imaging (Lahav et al. 1995; Abraham et al. 1996). The similar LFs derived from the SDSS Early Data Release (Nakamura et al. 2003) suggest that despite the improvement brought by CCD imaging, the visual morphological classification performed for this sample also suffers similar biases as in the nearby redshift surveys. Only the $B$ LF for the Stromlo-APM survey (Loveday et al. 1992, denoted SAPM) detects the Gaussian behavior for the E-S0 galaxies. This may however be due to the difficulty of classifying low surface brightness galaxies, and the possible resulting incompleteness of the SAPM classification in faint early-type galaxies.

Another noticeable result is that no existing redshift survey with morphological, spectral or color classification has measured the bounded LFs for the individual Spiral types ( $\mathrm{Sa}, \mathrm{Sb}, \mathrm{Sc}, \mathrm{Sd}$ ), nor the Gaussian shape of the LF for Spiral galaxies altogether, as measured locally (Sandage et al. 1985; Jerjen \& Tammann 1997). This confirms the interpretation of de Lapparent et al. (2003b), who show that the ESO-Sculptor Survey spectral-type LFs corresponding to Spiral galaxies might contain at their faint end a contribution from earlytype dwarf galaxies. Failure to separate the giant and dwarf galaxy populations in all existing redshift surveys, whatever the classification criterion, thus prevents any reliable measure of the Spiral intrinsic LFs. Even the 2 preliminary samples of the 2dFGRS (Folkes et al. 1999; Madgwick et al. 2002) and the commissioning data from the Sloan Digital Sky Survey (Blanton et al. 2001), which both sample with high statistical significance the galaxy distribution to $M(B) \simeq-16$, fail to measure the intrinsic Gaussian LF for Spiral galaxies.

The present analysis therefore emphasizes the need for more reliable and systematic approaches for morphological classification from $z \simeq 0$ to $z \gtrsim 1$, indispensable for measuring the intrinsic LFs, and their possible evolution with redshift. Morphological classification at $z \gtrsim 0.5$ is however a delicate task: it is wavelength dependent (Burgarella et al. 2001; Kuchinski et al. 2001), and detected evolution in galaxy morphology at $z \gtrsim 1$ complicates the definition of reference types (van den Bergh 1997; van den Bergh et al. 2000, 2001). A reliable discrimination among the morphological types is crucial for estimating intrinsic LFs, as these show a wide variety of shape and characteristic parameters for the different galaxy types (see Sect. 2 and Table 1). Inaccurate classification may then cause biases in the derived intrinsic LFs. In the analysis of the ESO-Sculptor Survey LFs, de Lapparent et al. (2003b) suggest that a useful morphological classification for measuring intrinsic LFs could include the surface brightness profile of the galaxies, as it allows to separate giant and dwarf galaxies, which have markedly different intrinsic LFs. So far, none of the existing redshift surveys provide separate LF measurements for the giant and dwarf galaxies.

Derivation of the intrinsic LFs per morphological type will also require redshift samples with at least $\sim 10^{5}$ galaxies, in order to have sufficient statistical samples of the various giant and dwarf galaxy types. Such large sample shall be obtained at $z \lesssim 0.2$ by the Sloan Digital Sky Survey (see http://www.sdss.org/), and the 2dF Galaxy Redshift
Survey (see http://www.mso.anu.edu.au/2dFGRS/). The preliminary LF measurements from these surveys which are analyzed here show that both the spectral classification used in the $2 \mathrm{dF}$ survey (based on a PCA spectral classification, Madgwick et al. 2002, and interpreted in terms of star formation history, Madgwick 2003), and the visual morphological classification used in the Early Data Release of the SDSS (Nakamura et al. 2003) appear insufficient for measurement of the intrinsic LFs. In contrast, the LFs derived from the SDSS commissioning data and based on 5 intervals of $g^{*}-r^{*}$ color succeed in detecting the Gaussian LF for the giant early-type galaxies (Blanton et al. 2001). Note that in view of the analyses presented here, the 2 color classes separated by $u^{*}-r *=2.22$ and shown by Strateva et al. (2001) to split SDSS galaxies according to morphological type and radial profile are likely to be insufficient to recover either the Gaussian LFs for the giant galaxies or the Schechter LFs for the dwarf galaxies. As I have shown here, measurement of LFs based on 2 color sub-samples lacks the necessary discriminatory power necessary for detecting the variations in luminosity as a function of morphological type which are traced by the intrinsic LFs.

I therefore recommend that in the case of multi-fiber surveys to moderate depths $\left(z_{\max } \lesssim 0.2\right)$, galaxy classification for estimation of the intrinsic LFs be based on rest-frame colors rather than on the spectral data. Whereas the fiber spectra only sample partial regions of the objects, the rest-frame colors do include the full light from the objects, and the accuracy of the photometric calibrations ensures that the colors reflect the shape of the SED for each object. A combined approach, which might yield improved results over a classification based on either multi-fiber spectroscopy or rest-frame color, is the calibration of spectral data using multi-color photometry, and its subsequent spectral classification. In the SDSS, the accurate photometry based on CCD multi-color imaging over 5 optical bands (Fukugita et al. 1996) should allow one to obtain such an improved classification. Performing such an analysis for $2 \mathrm{dF}$ Galaxy Redshift Survey, which is based on the APM scans of UK Schmidt $J$ photographic plates (Maddox et al. 1990b,a), with no color information, would require additional photometry in another band. Neither rest-frame colors nor spectral classification are however sufficient to separate the giant and dwarf galaxy populations and to measure the intrinsic LFs. To meet this goal, one additional step is the availability of the surface brightness profiles for all galaxies. A recent analysis of galaxy properties in a SDSS sample does use the radial profile of the objects (Blanton et al. 2002).

The DEEP2 (Davis et al. 2002) and VIRMOS (Le Fèvre et al. 2001) surveys, using deep CCD imaging and efficient multi-slit spectrographs on the Keck telescopes (Cowley et al. 1997; James et al. 1998) and ESO-VLT (Le Fèvre et al. 2001) resp., are also expected to bring useful measurements of intrinsic LFs at $z \sim 1$ and their possible evolution with redshift, provided that these surveys succeed in separating the various morphological types, including the giant and dwarf populations. So far, detection of evolution in the intrinsic LFs are based on either too few classes to allow a definite interpretation in terms of one morphological class (Lilly et al. 1995; Lin et al. 1999; Fried et al. 2001; de Lapparent et al. 2003b, using 2 and 3 classes), 
or on more classes but are suspected to suffer from type contamination (Heyl et al. 1997, see Sect. 3.3.3 above). Reliable analyses of the evolution in the intrinsic LFs with redshift will require the best resolution in morphological types.

I emphasize that an efficient technique for measuring LFs is the use of photometric redshifts: similar spectral type LFs are derived in the $B$ band for on one hand the CADIS (Fried et al. 2001) and COMBO-17 (Wolf et al. 2003) surveys, and on the other hand the CNOC2 (Lin et al. 1999) and ESO-Sculptor (de Lapparent et al. 2003b) surveys despite the significantly larger uncertainties in the redshifts derived from the CADIS and COMBO-17, measured from a combination of wide and medium-band filters $(\sigma(z) \leq 0.03)$. This demonstrates the interest of the "photometric redshift" approach for measuring LFs, which has the advantage of providing large samples at a reduced cost in telescope time. Along this line, 2 forthcoming surveys are expected to provide significant contributions to the measurement of the intrinsic LFs:

- the Large-Zenith-Telescope (LZT) project, which aims at obtaining redshifts for $\sim 10^{6}$ galaxies to $z \lesssim 1$ at $R \lesssim$ 23 over $40 \mathrm{deg}^{2}$ of the sky, using 40 medium-band filters (Cabanac et al. 2002; Hickson et al. 1998, see also http://www . astro. ubc.ca/LMT/lzt.html);

- the Canada-France-Hawaii Telescope Legacy Survey (see http://www . cfht .hawaii . edu/Science/CFHTLS/), which will cover $170 \mathrm{deg}^{2}$ in the wide-band filters $u^{*} g^{\prime} r^{\prime} i^{\prime} z^{\prime}$ at $r^{\prime} \lesssim 25.7$.

On one hand, the LZT will provide a detailed PCA-spectral classification and redshift measurements with an accuracy $\sigma(z) \leq 0.05$ at $z \lesssim 1$ (Cabanac et al. 2002), complemented by measures of surface brightness. On the other hand, by application of quantitative algorithms to the multi-color images obtained in excellent imaging conditions, the CFHT Legacy Survey will allow a detailed morphological classification, which shall be complemented by photometric redshifts with uncertainties $\sigma(z) / z \sim 0.1$ (Bolzonella et al. 2000). Both surveys will provide 2 orders of magnitude larger samples than the redshifts surveys, thus allowing useful measurements of the intrinsic LFs to $z \lesssim 1$.

I show here that the wide majority of intrinsic LF measurements were performed in the $B$ band, with few measurements in the $R_{\mathrm{c}}$ band, 2 measures in the $U$ band, a single one in the $V$ band, and no measurement in the $I_{\mathrm{c}}$ band. It will be important that the mentioned surveys under completion allow measurement of the intrinsic LFs in a variety of filters, including the ultraviolet and infrared. This will be a crucial step towards understanding the contribution from the different galaxy populations to the luminosity density of the Universe and for constraining its evolution with redshift.

Acknowledgements. I am grateful to Stéphane Arnouts for encouraging me to publish this analysis as a separate article, for suggesting its title, and for his useful comments on the article content. I also thank Josef Fried for providing me with the numerical values for the Schechter parameters of the CADIS luminosity functions (used in Table 4 and in Fig. 6), and Chris Wolf for providing the color corrections from the COMBO-17 filters into the Johnson system (used in Tables 2 and 3, and in Figs. 2 and 4).

\section{References}

Abraham, R. G., van den Bergh, S., Glazebrook, K., et al. 1996, ApJS, 107,1

Andreon, S., \& Cuillandre, J.-C. 2002, ApJ, 569, 144

Balcells, M., \& Peletier, R. F. 1994, AJ, 107, 135

Baugh, C. M., Benson, A. J., Cole, S., et al. 2002, in The Mass of Galaxies at Low and High Redshift, Venice 2001, ed. R. Bender, \& A. Renzini

Baugh, C. M., Cole, S., \& Frenk, C. S. 1996, MNRAS, 283, 1361

Beijersbergen, M., Hoekstra, H., van Dokkum, P. G., \& van der Hulst, T. 2002, MNRAS, 329, 385

Bellanger, C., de Lapparent, V., Arnouts, S., et al. 1995, A\&AS, 110, 159

Binggeli, B., \& Cameron, L. M. 1991, A\&A, 252, 27

Binggeli, B., \& Jerjen, H. 1998, A\&A, 333, 17

Binggeli, B., Sandage, A., \& Tammann, G. A. 1988, ARA\&A, 26, 509

Binggeli, B., Tarenghi, M., \& Sandage, A. 1990, A\&A, 228, 42

Blair, M., \& Gilmore, G. 1982, PASP, 94, 742

Blanton, M. R., Dalcanton, J., Eisenstein, D., et al. 2001, AJ, 121, 2358

Blanton, M. R., Hogg, D. W., Bahcall, N. A., et al. 2002, ApJ, submitted [astro-ph/0209479]

Blanton, M. R., Hogg, D. W., Bahcall, N. A., et al. 2003, ApJ, 592, 819

Bolzonella, M., Miralles, J.-M., \& Pelló, R. 2000, A\&A, 363, 476

Boroson, T. A., \& Thompson, I. B. 1987, AJ, 93, 33

Bromley, B. C., Press, W. H., Lin, H., \& Kirshner, R. P. 1998, ApJ, 505, 25

Brown, W. R., Geller, M. J., Fabricant, D. G., \& Kurtz, M. J. 2001, AJ, 122, 714

Burgarella, D., Buat, V., Donas, J., Milliard, B., \& Chapelon, S. 2001, A\&A, 369, 421

Cabanac, R. A., de Lapparent, V., \& Hickson, P. 2002, A\&A, 389, 1090

Charlot, S., \& Longhetti, M. 2001, MNRAS, 323, 887

Cole, S., Lacey, C. G., Baugh, C. M., \& Frenk, C. S. 2000, MNRAS, 319, 168

Coleman, G. D., Wu, C., \& Weedman, D. W. 1980, ApJS, 43, 393

Collins, C. A., Heydon-Dumbleton, N. H., \& MacGillivray, H. T. 1989, MNRAS, 236, 7

Conselice, C. J., Gallagher, J. S., \& Wyse, R. F. G. 2002, AJ, 123, 2246

Cowley, D. J., Faber, S., Hilyard, D. F., James, E., \& Osborne, J. 1997, in Optical Telescopes of Today and Tomorrow, ed. A. L. Ardeberg, Proc SPIE, 2871, 1107

Davis, M., Faber, S. M., Newman, J. A., et al. 2002, in Proc. SPIE, 4841

Davis, M., Huchra, J., Latham, D. W., \& Tonry, J. 1982, ApJ, 253, 423 de Lapparent, V., Arnouts, S., \& Galaz, G. 2003a, A\&A, in preparation de Lapparent, V., Galaz, G., Bardelli, S., \& Arnouts, S. 2003b, A\&A, 404, 831

de Lapparent, V., Geller, M. J., \& Huchra, J. P. 1986, ApJ, 302, L1

de Vaucouleurs, G. 1948, Ann. Astrophys., 11, 247

de Vaucouleurs, G., de Vaucouleurs, A., Corwin, H. G., et al. 1991, Third Reference Catalogue of Bright Galaxies, Vols. 1-3, XII (Berlin, Heidelberg, New York: Springer-Verlag)

Dressler, A. 1980, ApJ, 236, 351

Driver, S. P., Phillipps, S., Davies, J. I., Morgan, I., \& Disney, M. J. 1994, MNRAS, 268, 393

Durret, F., Adami, C., Gerbal, D., \& Pislar, V. 2000, A\&A, 356, 815

Efstathiou, G., Ellis, R. S., \& Peterson, B. A. 1988, MNRAS, 232, 431 
Ellis, R. S., Colless, M., Broadhurst, T., Heyl, J., \& Glazebrook, K. 1996, MNRAS, 280, 235

Ferguson, H. C. 1989 , AJ, 98, 367

Ferguson, H. C., \& Sandage, A. 1991, AJ, 101, 765

Fioc, M., \& Rocca-Volmerange, B. 1997, A\&A, 326, 950

Flint, K., Bolte, M., \& Mendes de Oliveira, C. 2001a, in Dwarf galaxies and their environment, 209

Flint, K., Metevier, A. J., Bolte, M., \& Mendes de Oliveira, C. 2001b, ApJS, 134, 53

Folkes, S., Ronen, S., Price, I., et al. 1999, MNRAS, 308, 459

Fried, J. W., von Kuhlmann, B., Meisenheimer, K., et al. 2001, A\&A, 367,788

Fukugita, M., Ichikawa, T., Gunn, J. E., et al. 1996, AJ, 111, 1748

Fukugita, M., Shimasaku, K., \& Ichikawa, T. 1995, PASP, 107, 945

Galaz, G., \& de Lapparent, V. 1998, A\&A, 332, 459

Garilli, B., Maccagni, D., \& Andreon, S. 1999, A\&A, 342, 408

Gaztañaga, E., \& Dalton, G. B. 2000, MNRAS, 312, 417

Geller, M. J., Kurtz, M. J., Wegner, G., et al. 1997, AJ, 114, 2205

Heyl, J., Colless, M., Ellis, R. S., \& Broadhurst, T. 1997, MNRAS, 285,613

Hickson, P., Borra, E. F., Cabanac, R., et al. 1998, Proc. SPIE, 3352, 226

Hilker, M., Mieske, S., \& Infante, L. 2003, A\&A, 397, L9

James, E. C., Cowley, D. J., Faber, S. M., Hilyard, D. F., \& Osborne, J. 1998, in Optical Astronomical Instrumentation, ed. S. D’Odorico, Proc. SPIE, 3355, 70

Jerjen, H., Binggeli, B., \& Freeman, K. C. 2000, AJ, 119, 593

Jerjen, H., \& Tammann, G. A. 1997, A\&A, 321, 713

Kambas, A., Davies, J. I., Smith, R. M., Bianchi, S., \& Haynes, J. A. 2000, AJ, 120, 1316

Kauffmann, G., \& Charlot, S. 1998, MNRAS, 297, L23

Kauffmann, G., Nusser, A., \& Steinmetz, M. 1997, MNRAS, 286, 795

Kennicutt, R. C. 1992a, ApJS, 79, 255

Kennicutt, R. C. 1992b, ApJ, 388, 310

Kinney, A. L., Calzetti, D., Bohlin, R. C., et al. 1996, ApJ, 467, 38

Kochanek, C. S., Pahre, M. A., \& Falco, E. E. 2001 [astro-ph/0011458]

Kuchinski, L. E., Madore, B. F., Freedman, W. L., \& Trewhella, M. 2001, AJ, 122, 729

Lahav, O., Naim, A., Buta, R. J., et al. 1995, Sci, 267, 859

Lasker, B. M., Sturch, C. R., McLean, B. J., et al. 1990, AJ, 99, 2019

Lauberts, A., Holmberg, E. B., Schuster, H.-E., \& West, R. M. 1981, A\&AS, 46, 311

Le Fèvre, O., Vettolani, G., Maccagni, D., et al. 2001, in Deep Fields, 236

Lilly, S. J., Tresse, L., Hammer, F., Crampton, D., \& Le Fevre, O. 1995, ApJ, 455, 108

Lin, H., Kirshner, R. P., Shectman, S. A., et al. 1996, ApJ, 464, 60

Lin, H., Yee, H. K. C., Carlberg, R. G., \& Ellingson, E. 1997, ApJ, 475, 494

Lin, H., Yee, H. K. C., Carlberg, R. G., et al. 1999, ApJ, 518, 533

Loveday, J., Maddox, S. J., Efstathiou, G., \& Peterson, B. A. 1995, ApJ, 442, 457

Loveday, J., Peterson, B. A., Efstathiou, G., \& Maddox, S. J. 1992, ApJ, 390, 338

Loveday, J., Tresse, L., \& Maddox, S. 1999, MNRAS, 310, 281

Maddox, S. J., Efstathiou, G., \& Sutherland, W. J. 1990a, MNRAS, 246,433
Maddox, S. J., Efstathiou, G., Sutherland, W. J., \& Loveday, J. 1990b, MNRAS, 243, 692

Madgwick, D. S. 2003, MNRAS, 338, 197

Madgwick, D. S., Lahav, O., Baldry, I. K., et al. 2002, MNRAS, 333, 133

Marinoni, C., Monaco, P., Giuricin, G., \& Costantini, B. 1999, ApJ, 521, 50

Marzke, R. O., da Costa, L. N., Pellegrini, P. S., Willmer, C. N. A., \& Geller, M. J. 1998, ApJ, 503, 617

Marzke, R. O., Geller, M. J., Huchra, J. P., \& Corwin, H. G. 1994a, AJ, 108, 437

Marzke, R. O., Huchra, J. P., \& Geller, M. J. 1994b, ApJ, 428, 43

Metcalfe, N., Ratcliffe, A., Shanks, T., \& Fong, R. 1998, MNRAS, 294, 147

Mobasher, B., Colless, M., Carter, D., et al. 2003, ApJ, 587, 605

Naim, A., Lahav, O., Buta, R. J., et al. 1995, MNRAS, 274, 1107

Nakamura, O., Fukugita, M., Yasuda, N., et al. 2003, AJ, 125, 1682

Nilson, P. 1973, Nova Acta Regiae Soc. Sci. Upsaliensis Ser. V, 0

Norberg, P., Cole, S., Baugh, C. M., et al. 2002, MNRAS, 336, 907

Postman, M., \& Geller, M. J. 1984, ApJ, 281, 95

Pritchet, C. J., \& van den Bergh, S. 1999, AJ, 118, 883

Ramella, M., Geller, M. J., \& Huchra, J. P. 1990, ApJ, 353, 51

Ramella, M., Geller, M. J., Pisani, A., \& da Costa, L. N. 2002, AJ, 123, 2976

Ratcliffe, A., Shanks, T., Parker, Q. A., \& Fong, R. 1998, MNRAS, 293, 197

Sandage, A., Binggeli, B., \& Tammann, G. A. 1985, AJ, 90, 1759

Schechter, P. 1976, ApJ, 203, 297

Schroeder, A., \& Visvanathan, N. 1996, A\&AS, 118, 441

Segalovitz, A. 1975, A\&A, 40, 401

Small, T. A., Sargent, W. L. W., \& Hamilton, D. 1997, ApJ, 487, 512

Strateva, I., Ivezić, Ž., Knapp, G. R., et al. 2001, AJ, 122, 1861

Thuan, T. X., \& Gunn, J. E. 1976, PASP, 88, 543

Tonry, J., \& Davis, M. 1979, AJ, 84, 1511

Trentham, N. 1998, MNRAS, 294, 193

Trentham, N., \& Hodgkin, S. 2002, MNRAS, 333, 423

Trentham, N., \& Tully, R. B. 2002, MNRAS, 335, 712

Tresse, L., Maddox, S., Loveday, J., \& Singleton, C. 1999, MNRAS, 310,262

Treyer, M. A., Ellis, R. S., Milliard, B., Donas, J., \& Bridges, T. J. 1998, MNRAS, 300, 303

Valotto, C. A., Moore, B., \& Lambas, D. G. 2001, ApJ, 546, 157 van den Bergh, S. 1997, AJ, 113, 2054

van den Bergh, S., Cohen, J. G., \& Crabbe, C. 2001, AJ, 122, 611

van den Bergh, S., Cohen, J. G., Hogg, D. W., \& Blandford, R. 2000, AJ, 120, 2190

Vigroux, L., Souviron, J., Lachieze-Rey, M., \& Vader, J. P. 1988, A\&AS, 73, 1

Wilson, G., Smail, I., Ellis, R. S., \& Couch, W. J. 1997, MNRAS, 284, 915

Wolf, C. 2002, private communication

Wolf, C., Meisenheimer, K., Rix, H.-W., et al. 2003, A\&A, 401, 73

Yagi, M., Kashikawa, N., Sekiguchi, M., et al. 2002, AJ, 123, 87

Zucca, E., Zamorani, G., Vettolani, G., et al. 1997, A\&A, 326, 477

Zwicky, F., Herzog, E., \& Wild, P. 1968, Catalogue of galaxies and of clusters of galaxies (Pasadena: California Institute of Technology (CIT), 1961-1968) 